\title{
Research Progression of the Genus Merremia: A Comprehensive Review on the Nutritional Value, Ethnomedicinal Uses, Phytochemistry, Pharmacology, and Toxicity
}

\author{
Tomi Lois Olatunji ${ }^{1, *} \mathbb{D}^{\mathbb{D}}$, Ademola Emmanuel Adetunji ${ }^{2}{ }^{(D}$, Chijioke Olisah $^{3}\left(\mathbb{D}\right.$, Oladayo Amed Idris $^{1}{ }^{(}$, \\ Oluwaseyi Damilare Saliu ${ }^{4}\left(\mathbb{D}\right.$ and Frances Siebert ${ }^{1}$ (1) \\ 1 Unit for Environmental Sciences and Management (UESM), Faculty of Natural and Agricultural \\ Sciences, North-West University, Potchefstroom 2520, South Africa; dayoamed@yahoo.com (O.A.I.); \\ frances.siebert@nwu.ac.za (F.S.) \\ 2 School of Life Sciences, University of KwaZulu-Natal, Durban 4001, South Africa; \\ adetunjiademola@hotmail.com \\ 3 Department of Botany, Institute for Coastal and Marine Research, Nelson Mandela University, \\ Port Elizabeth 6031, South Africa; olisah.chijioke@gmail.com \\ 4 Department of Chemical Sciences, University of Johannesburg, Johannesburg 2028, South Africa; \\ oluwaseyi229@gmail.com \\ * Correspondence: lois.olatunji@gmail.com
}

\section{check for}

updates

Citation: Olatunji, T.L.; Adetunji, A.E.; Olisah, C.; Idris, O.A.; Saliu,

O.D.; Siebert, F. Research Progression

of the Genus Merremia:

A Comprehensive Review on the

Nutritional Value, Ethnomedicinal Uses, Phytochemistry, Pharmacology, and Toxicity. Plants 2021, 10, 2070 https://doi.org/10.3390/plants10102070

Academic Editors

Mariangela Marrelli and Luigi Milella

Received: 16 August 2021

Accepted: 24 September 2021

Published: 30 September 2021

Publisher's Note: MDPI stays neutral with regard to jurisdictional claims in published maps and institutional affiliations.

Copyright: (c) 2021 by the authors. Licensee MDPI, Basel, Switzerland This article is an open access article distributed under the terms and conditions of the Creative Commons Attribution (CC BY) license (https:// creativecommons.org/licenses/by/ $4.0 /)$
Abstract: The genus Merremia Dennst. ex Endl. (Convolvulaceae) is a rich source of structurally diverse phytochemicals with therapeutic relevance. This review presents the first comprehensive, up-to-date information and research progression on the nutritional value, ethnomedicinal uses, phytochemistry, pharmacological activities, and toxicity of the genus Merremia. Using the key search term "Merremia", relevant documents and information were retrieved from electronic databases. Relevant documents were uploaded in RStudio with installed bibliometric software packages and used for data retrieval, tabulation, and network visualization. Bibliometric analysis revealed that ca. $55 \%$ of the studies related to Merremia were published in the last decade, which can be grouped into four thematic areas: (i) drug formulation, (ii) taxonomy, (iii) chemical analysis, and (iv) treatment of diseases. Ethnomedicinal uses, phytochemistry, and biological activities studies showed that species in the genus are promising medicinal plants with various pharmaceutical potentials. However, clinical studies to validate the efficacy of the reported bioactivities and the mechanisms underlying the various activities are lacking and should constitute a future research focus. Additionally, reports on the nutritional and antinutritional constituents of Merremia species revealed that the species meet high nutritional quality criteria for animals and are therefore suitable for inclusion in livestock diets. The few available investigations on toxicity indicated that most Merremia species are safe for human and animal use but not with prolonged chronic administration.

Keywords: bibliometric; Convolvulaceae; fodder; Merremia; merremins; resin glycosides

\section{Introduction}

Natural medicines, whether from standardized plant extracts or pure compounds from plants, are valuable sources of new drugs in the pharmaceutical industries because of their diverse biologically active phytochemicals [1,2]. Over the years, medicinal plants have been used in many human populations all over the world [3] in the treatment of different ailments and diseases. This is largely due to their health benefits, effectiveness [4], affordability, and little or no side effects when compared to synthetic drugs that are expensive and may have adverse effects [5].

A large proportion of species used in traditional medicine globally are forbs (syn. herbs), which are one of the most species-rich plant life forms, especially in open ecosystems worldwide [6,7]. Several indigenous rangeland forb species are consumed as vegetables, 
thus forming a part of local people's diet. They are economically useful crops that had bolstered food security historically when the main crops failed, and thus should be increased in production to solve the global food crises [8]. Forbs are important nutritive dietary components of several browsers as components of mixed pastures and have been reported to contain higher levels of phosphorus and crude protein, and lower fiber levels than shrubs or grasses [6]. Additionally, many of the forb species are used in traditional medicine to treat different ailments, and the broad range of bioactive phytochemicals present in some forb species can be potentially employed for the treatment and prevention of several diseases in modern medicine. Of these forbs, species in the genus Merremia have been the focus of several pharmacological and phytochemical studies.

Merremia Dennst. ex Endl. is a large genus of over 100 species of flowering plants in the Convolvulaceae (morning glory) family [9]. Members of the family Convolvulaceae are usually recognized by their colorful funnel-shaped flowers [10,11]. Resin glycosides [12,13], calystegines [14], and tropane alkaloids [15] are considered the chemotaxonomic markers of the Convolvulaceae family.

The genus Merremia, commonly referred to as wood roses, is distributed in tropical and sub-tropical regions all over the world [16]. Several species in the genus are of reputed medicinal value and are used in traditional medicine across continents. For example, in Sri Lanka, the whole plant of M. emarginata is prepared as a decoction and used in the traditional treatment of diabetes [17]. In India, leaves of $M$. hederacea have been reported to be used in treating chapped hands and feet [18]. In the Philippines, the leaves of M. peltata are used for treating skin sores, inflammation, and stomach pain [19]. The leaves and rhizomes of $M$. vitifolia are used in the traditional treatment of eye inflammation, dysentery, urinary diseases, and jaundice in Bangladesh [20]. It is also evident from the literature that the genus Merremia is a rich source of biologically active phytochemicals [21-23] since extracts from the different species have shown pharmacological activities in several in vitro and in vivo studies. Thus, species in the genus Merremia would be promising alternative sources of new pharmaceutical leads in treating several diseases and ailments. Despite the widespread ethnopharmacological importance and different scientific investigations of phytochemistry and pharmacological activities, there is no comprehensive review analyzing, documenting, and revealing the pharmacological importance of this genus.

Bibliometric analysis has been recognized as a useful statistical method that can qualitatively and quantitatively evaluate the trend in research efforts in a given area of interest $[24,25]$. Bibliometrics can thus be employed to assess both national and international research focus and is particularly useful in providing insights for future research [26].

This review presents the first comprehensive, up-to-date information and research progression on the nutritional value, ethnomedicinal uses, phytochemistry, and pharmacological activities of the species in the genus Merremia. Hence, this review is aimed to (i) identify the international research focus of the genus, (ii) provide insight into the emerging pharmacological potentials of Merremia species, (iii) highlight biological activities of extracts and isolated phytochemicals, (iv) highlight its potentials as livestock forage and (v) provide the scientific basis for further research on the nutritional value, pharmacological potentials and their mainstream application.

\section{Materials and Methods}

For the global bibliometric analysis of the genus Merremia, document retrieval and statistical analysis were conducted based on the method described in $[27,28]$. In summary, document information related to Merremia (between 1990-2020) was retrieved from 10 databases indexed in the Web of Science (WoS) ("SCI EXPANDED, SSCI, A\&HCI, CPCI-S, CPCI-SSH, BKCI-S, BKCI-SSH, ESCI, CCR EXPANDED, and IC"). The term "Merremia" was inputted as a search term in the topic module on the WoS database hosted in Clarivate Analytics as well as in the Scopus topical search engine. Only document types including "Article", "Review," "Editorial Material", and "Book Chapter" were searched. Other document types such as "Meeting Abstract", "Proceeding Paper", and "Notes" 
were excluded. A total of 194 documents satisfied the search criteria in the WoS database. The total search yielded a total of 261 publications from the Scopus database. It should be noted that the documents excluded from both databases were because they were either pre-publication data that may have been published as an article or post-article data taken from the primary articles [26,27]. The identified number of articles from both databases were downloaded in Bibtex and uploaded in RStudio for further statistical processing.

RStudio Inc. (Version 1.1.463- C 2021-2018, Boston, MA, USA) with installed bibliometric software packages was used for data retrieval, tabulation, and network visualization. Duplicate records from the hybrid databases were merged using $\mathrm{R}$ codes, and these were taken as a single article. A total number of 357 publications were used for bibliometric analysis. Commands for analyzing bibliometric indicators were retrieved from https: / / cran.rproject.org/web/packages/bibliometrix/vignettes/bibliometrix-vignette.html, accessed on 15 February 2021.

Keyword network map was visualized using VOS viewer (Nees Jan van Eck and Ludo Waltman; Leiden; The Netherlands, version 1.6.15- C 2021-2020).

Information on the nutritional value, ethnomedicinal uses, phytochemistry, biological activities, and toxicity studies were retrieved from relevant articles, books, webpages and other online materials searched using different databases. The search period was from inception to July 2021 in the different databases. The Boolean string used was "Merremia", searching the "title", "abstract", "authors keyword", and "keyword plus" of the documents. The search was limited to materials available in English. The relevant materials retrieved were used to present a comprehensive and up-to-date review write-up on the subject matter. All species names and synonyms were confirmed from the "World Flora Online (WFO)". All chemical structures were drawn with Chem Draw.

\section{Results}

\subsection{Bibliometric Overview of the Genus-Merremia}

A total of 357 documents with an average citation per article of 10.39 was retrieved from the merged databases (WoS and Scopus) (Table 1). Of all the documents present, 341 were research articles and 12 review articles. Other document types included book chapters and editorial materials, which revealed two documents in each. Documents were obtained from 188 journals and included 1005 author keywords and 2727 keywords plus. There was a slightly steady increase in documents produced between 1990 and 2020 with a major fluctuation in the first (1990-2000) and last (2011-2020) decades of the survey period. Peak numbers were recorded in 2009 with 33 articles, followed by 2020 and 2010 with 26 and 25 articles, respectively. Approximately 55\% of the articles published on studies related to Merremia were published in the last decade (2011-2020). A high number of articles in these years may be due to the availability of funds, the advent of research ideas, and the emergence of sophisticated analytical tools for chemical analysis.

Table 1. Summarized bibliographic data.

\begin{tabular}{cc}
\hline Bibliographic Information & Numerical Outputs \\
\hline Publications & 357 \\
Sources (Journals, Books, etc.) & 188 \\
Keywords Plus (ID) & 2727 \\
Author's Keywords (DE) & 1005 \\
Period & $1990-2020$ \\
Average citations per document & 10.39 \\
Authors & 948 \\
Author Appearances & 1537 \\
Authors of multi-authored documents & 937 \\
Single-authored documents & 18 \\
Average documents per author & 0.377 \\
Average authors per document & 2.66 \\
Average co-authors per document & 4.31 \\
Average collaboration index & 2.76 \\
\hline
\end{tabular}




\subsection{Most Productive Countries}

To avoid multiple representations of authors, authors from the same country in an article were computed as one. We prioritized this bibliometric tool to recognize the most prolific countries on Merremia related research. As shown in Table 2, authors' countries were ranked based on the number of articles and citations accumulated over the survey period. Brazil had the highest number of articles $(n=116)$, with $32.5 \%$ of the total number of articles. This was followed by India $(n=51,14.3 \%)$, Japan $(n=27,7.6 \%)$, USA $(n=26,7.3 \%)$, and China $(n=18,5.0 \%)$. Based on continental production, Asia and America were more prolific, having seven countries each in the top 20 authors' countries on studies related to the genus Merremia. Most of the Merremia species are found in the Asian continent, which may be responsible for their high number of articles (https:/ /www.cabi.org/isc/ datasheet/33477, accessed on 23 March 2021). As for the Americas, the United States Department of Agriculture (USDA) plants database reported that seven species of Merremia occur in North America. Four of these species remain unique to the North American region, while three have been neutralized in other parts of the world. These species include M. quinquefolia, M. cissoids, and M. umbellata that are native to Florida, while M. dissecta (Alamo vine) occurs in Texas, Pennsylvania, and states in the south-eastern region (https:/ / www.wildflower.org/expert/show.php?id=7618, accessed on 28 March 2021). A country that hosts a substantial number of studied species is expected to attract more scientific research compared to other countries [29], which explains why the USA is ranked fourth in the total number of article outputs on Merremia. The species M. aegyptia is a native of South Africa only, which may explain why South Africa is the only African country listed under the top 20 most productive countries with respect to Merremia research.

Table 2. Top 20 authors' countries on studies related to the genus-Merremia from 1990-2020. SCP—single country publications. $\mathrm{MCP}-$ multiple country publications. $\mathrm{MCP} / \mathrm{P}-$ multiple country publications per publication.

\begin{tabular}{|c|c|c|c|c|c|c|c|c|c|c|c|}
\hline \multicolumn{8}{|c|}{ Most Productive Countries } & \multicolumn{4}{|c|}{ Total Number of Citations per Country } \\
\hline Rank & Countries & Articles & $\begin{array}{l}\% \% \text { of } \\
\text { Total }\end{array}$ & Freq & SCP & MCP & $\begin{array}{c}\mathrm{MCP} / \mathrm{P} \\
\text { Ratio }\end{array}$ & Rank & Country & $\begin{array}{c}\text { Article } \\
\text { Citations }\end{array}$ & $\begin{array}{l}\text { Citation } \\
\text { Average }\end{array}$ \\
\hline 1 & Brazil & 116 & 32.5 & 0.34 & 112 & 4 & 0.03 & 1 & India & 769 & 15.1 \\
\hline 2 & India & 51 & 14.3 & 0.15 & 49 & 2 & 0.04 & 2 & Brazil & 697 & 6.0 \\
\hline 3 & Japan & 27 & 7.6 & 0.08 & 26 & 1 & 0.04 & 3 & Japan & 536 & 19.9 \\
\hline 4 & USA & 26 & 7.3 & 0.08 & 19 & 7 & 0.27 & 4 & Germany & 399 & 30.7 \\
\hline 5 & China & 18 & 5.0 & 0.05 & 16 & 2 & 0.11 & 5 & USA & 295 & 11.4 \\
\hline 6 & Germany & 13 & 3.6 & 0.04 & 10 & 3 & 0.23 & 6 & Mexico & 173 & 17.3 \\
\hline 7 & Indonesia & 13 & 3.6 & 0.04 & 13 & 0 & 0.00 & 7 & Malaysia & 117 & 29.3 \\
\hline 8 & Mexico & 10 & 2.8 & 0.03 & 8 & 2 & 0.20 & 8 & China & 102 & 5.7 \\
\hline 9 & Belgium & 6 & 1.7 & 0.02 & 3 & 3 & 0.50 & 9 & Panama & 89 & 22.3 \\
\hline 10 & Venezuela & 6 & 1.7 & 0.02 & 5 & 1 & 0.17 & 10 & Spain & 77 & 25.7 \\
\hline 11 & Australia & 5 & 1.4 & 0.01 & 4 & 1 & 0.20 & 11 & Colombia & 67 & 22.3 \\
\hline 12 & $\begin{array}{l}\text { South } \\
\text { Africa }\end{array}$ & 5 & 1.4 & 0.01 & 5 & 0 & 0.00 & 12 & $\begin{array}{l}\text { South } \\
\text { Africa }\end{array}$ & 53 & 10.6 \\
\hline 13 & Malaysia & 4 & 1.1 & 0.01 & 4 & 0 & 0.00 & 13 & Canada & 41 & 20.5 \\
\hline 14 & Panama & 4 & 1.1 & 0.01 & 2 & 2 & 0.50 & 14 & Australia & 40 & 8.0 \\
\hline 15 & Argentina & 3 & 0.8 & 0.01 & 2 & 1 & 0.33 & 15 & Argentina & 36 & 12.0 \\
\hline 16 & Colombia & 3 & 0.8 & 0.01 & 1 & 2 & 0.67 & 16 & Belgium & 31 & 5.2 \\
\hline 17 & Spain & 3 & 0.8 & 0.01 & 1 & 2 & 0.67 & 17 & Indonesia & 28 & 2.2 \\
\hline 18 & Thailand & 3 & 0.8 & 0.01 & 3 & 0 & 0.00 & 18 & Venezuela & 26 & 4.3 \\
\hline 19 & $\begin{array}{l}\text { United } \\
\text { Kingdom }\end{array}$ & 3 & 0.8 & 0.01 & 1 & 2 & 0.67 & 19 & Togo & 22 & 22.0 \\
\hline 20 & Bangladesh & 2 & 0.6 & 0.01 & 2 & 0 & 0.00 & 20 & Thailand & 17 & 5.7 \\
\hline
\end{tabular}

\subsection{Keyword Analysis}

Keywords reflect the core content of an article, and its analysis in this study aims to recognize the evolving research topic. Figure 1 shows the keyword network generated in the VOS viewer. The keyword network map generated shows the major thematic areas in the literature. Colored clusters represent thematic domains, while words enclosed in large dotted nodes represent the most frequently used terms. Lines between terms show the frequency of occurrence in articles. The map reveals that studies related to Merremia 
species can be grouped into four thematic areas-Drug formulation, taxonomy, chemical analysis, and treatment of diseases.

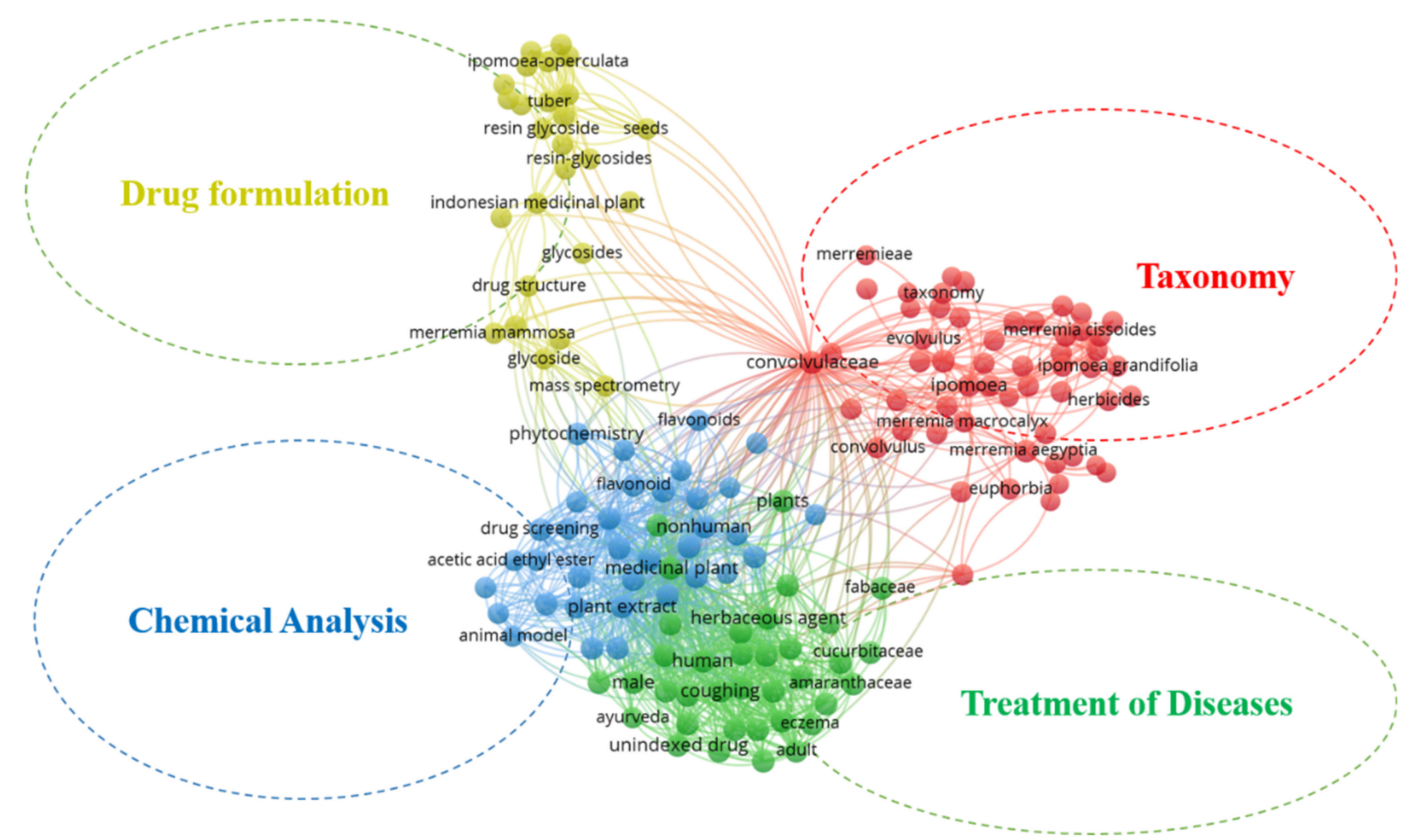

Figure 1. Top keyword co-occurrence networks based on research articles on Merremia. Colored clusters represent different research fields, while terms enclosed in colored squares represent the most frequently used keywords.

\subsection{Genus Merremia}

\subsubsection{Taxonomy, Botanical Description, and Distribution}

The genus Merremia has been reported to be a polyphyletic genus with strong evidence from different molecular analyses [11,30,31]. The taxonomic classification of the genus is provided in Table 3 Approximately 182 species, including infraspecific names, have been reported to belong to the genus Merremia, although only 71 correspond to an accepted name, of which 38 are synonyms and 73 listed as unresolved on the Plant List database [32].

Table 3. Taxonomic classification of the genus Merremia.

\begin{tabular}{cc}
\hline Domain & Eukaryota \\
Kingdom & Plantae \\
Phylum & Spermatophyta \\
Subphylum & Angiospermae \\
Class & Dicotyledonae \\
Order & Solanales \\
Family & Convolvulaceae \\
Genus & Merremia \\
\hline
\end{tabular}

Merremia species are mostly shrubs or herbs (i.e., herbaceous forbs) with a generally twining or prostrate, but rarely erect growth form. The leaves are entire, lobed, or compound with 3-7 leaflets. Flowers are axillary and solitary or in few- to manyflowered cymes. Bracts are small, ovate or elliptic, and linear. Sepals five, usually subequal, elliptical ovate or ovate-oblong. Corollas are campanulate or funnel-shaped, lobed or entire, yellow or white, sometimes with a dark brown or purple center. Stamens five, filaments filiform, subequal or equal, and are usually widened and glabrous at the base. The anthers are usually twisted with full dehiscence. Pollen grains are smooth and can be 3, 5-6, 9-12-colpate, or 12-rugate. Ovary 2-4-celled, usually with 4 ovules; style filiform; 
stigmas 2, which are globose or biglobose. Fruits are capsules, usually four-valved that dehisce longitudinally. The seeds are usually 4-6 in number, pubescent, or glabrous.

Conventionally, species in the genus are recognized by white or yellow flowers with two globos stigmas, anthers that are twisted when flowers are fully opened and functional, and non-spiny pollen grains that could be zonocolpate, tricolpate, pantoporate, pantocolpate, non-spinulose, and valvate fruit dehiscence [10,33] (Figure 2).

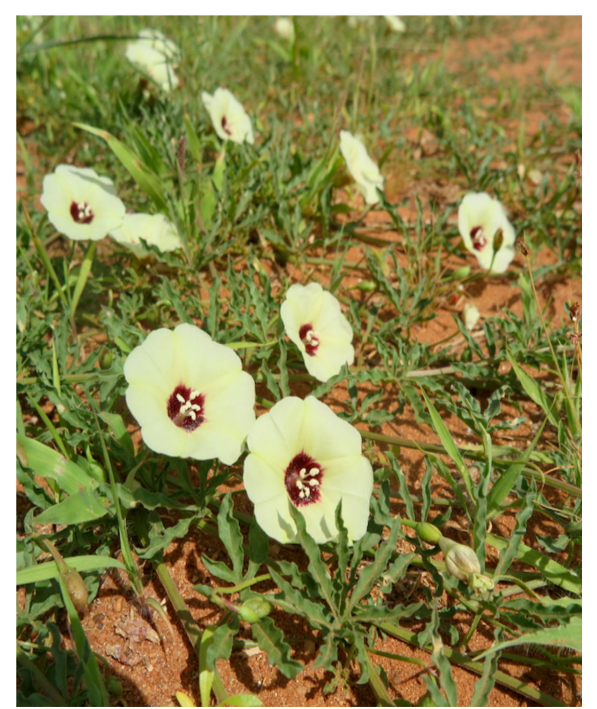

(a)

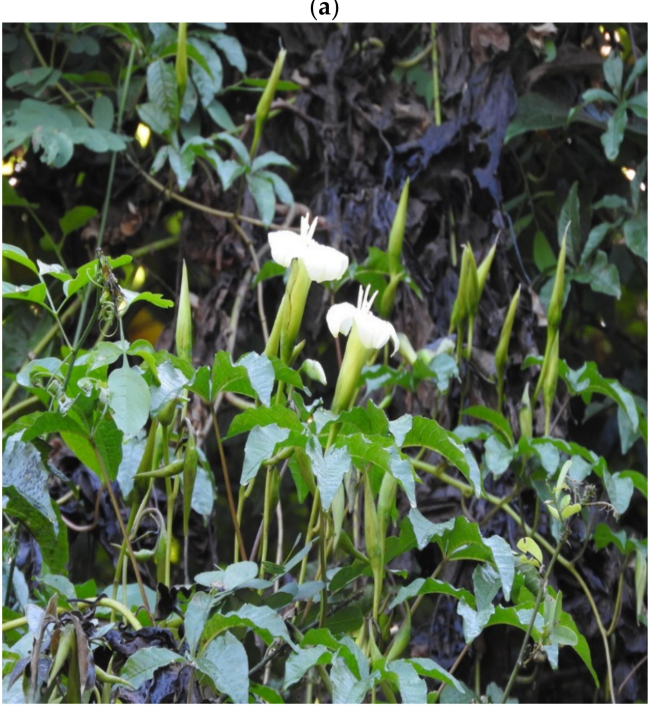

(c)

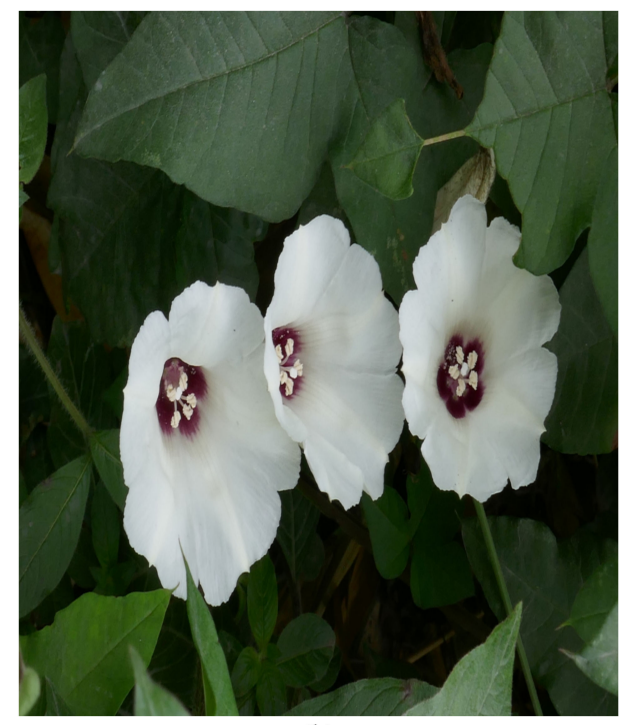

(b)

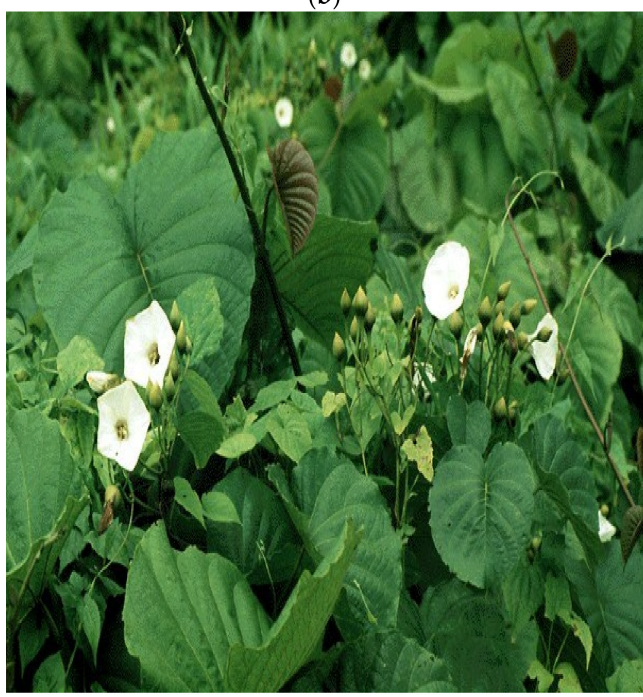

(d)

Figure 2. Cont. 


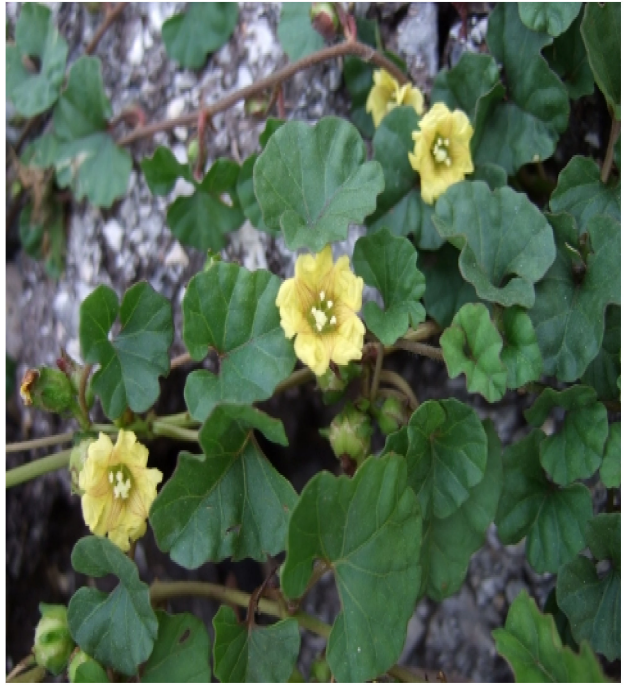

(e)

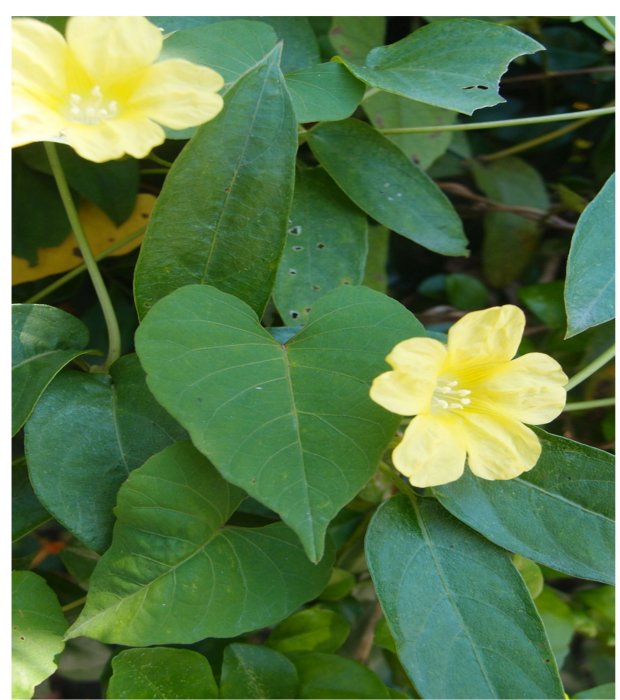

(f)

Figure 2. Images of some Merremia species. (a) Merremia palmata Image: () Riana Fourie, (CC-BYNC) [34]. (b) Merremia pterygocaulos Image: (C) Thierrycordenos, (CC-BY-NC) [35]. (c) Merremia platylphylla Image: (c) Alexis López Hernández [36]. (d) Merremia peltata Image: (c) Matthew Cock [37]. (e) Merremia emarginata Image: (C) Convolvulaceae unlimited [38]. (f) Merremia gemella Image: (c) Wan-hsuan [39].

Merremia species are distributed in tropical and subtropical regions around the world. Merremia is native to Africa, Asia, Australia, North America, and South America. Its full distribution listing is shown in Figure 3 and Supplementary Table S1.

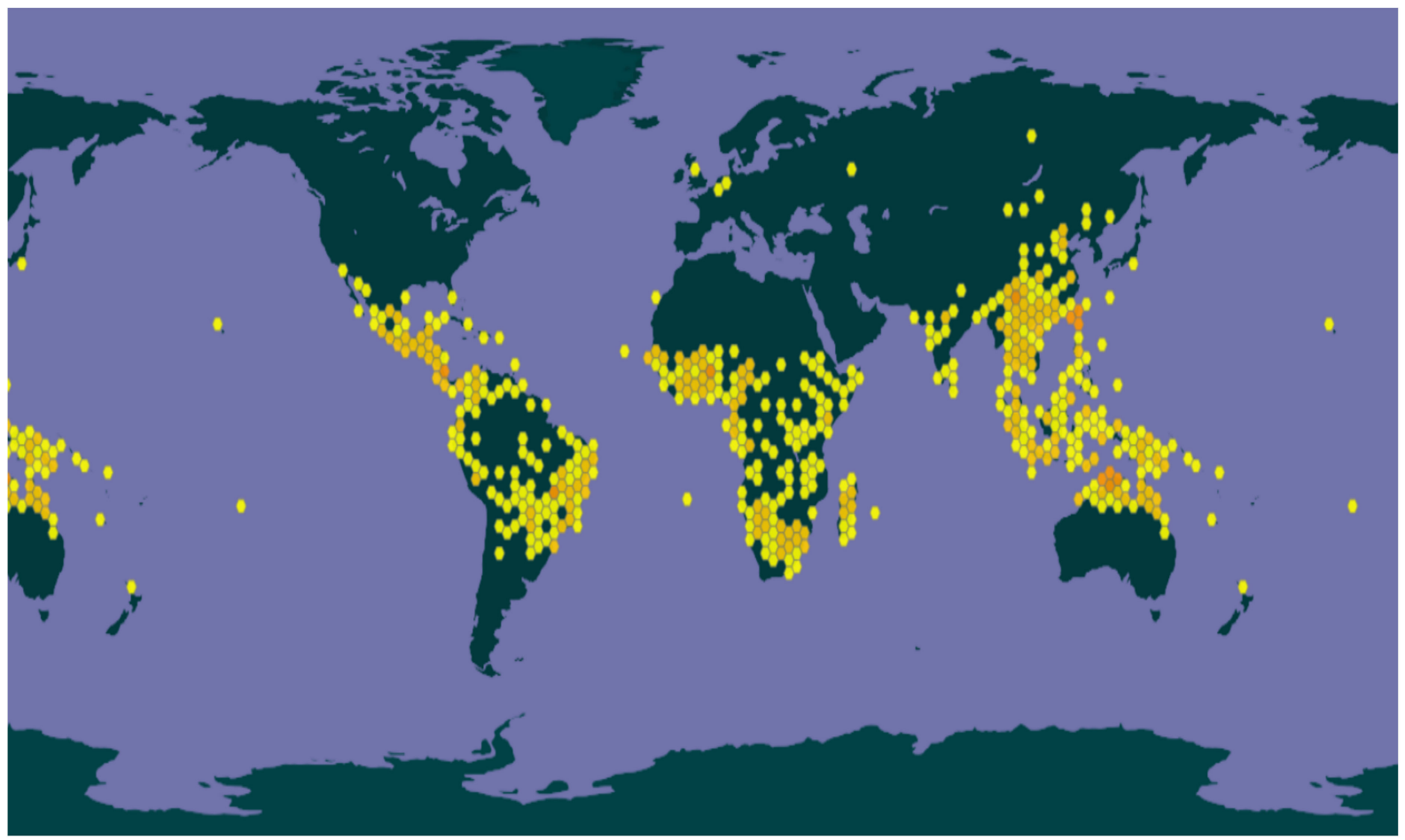

Figure 3. Global distribution of Merremia species (Image @ OpenStreetMap contributors, GBIF, @ OpenMapTiles) [40]. 


\subsubsection{Nutritional Value of Merremia Species}

Some Merremia species are a good source of food and are particularly useful as important fodders for livestock feed. The whole plant of $M$. emarginata is famous for salad in Central Myanmar, Korea [41]. In Argentina, the roots of $M$. dissecta var. edentata are used for food by some indigenous people [42]. The leaves of $M$. tridentata are cooked as vegetables in Guinea-Bissau, West Africa [43]. The nutritional analysis of the whole plant of M. emarginata revealed important nutritional constituents, which included carbohydrate $(63.10 \%)$ fat $(1.05 \%)$, fiber $(15.55 \%)$, protein $(3.28 \%)$, ash $(7.29 \%)$, and moisture $(9.73 \%)$ [41] Nunes et al. [44] carried out an ethnobotanical survey of plants used as animal forage in two rural communities in north-eastern Brazil and determined their nutritional and anti-nutritional constituents. Merremia aegyptia was listed among the forage plants with a local preference for feeding ruminants given its promotive effect in terms of weight gain and milk production. The analysis of the nutritional composition revealed that $M$. aegyptia has good potential for use in ruminant diets in terms of crude protein contents $(20.33 \pm 6.26 \%)$ and mineral matter $(14.06 \%)$. The anti-nutritional analysis further suggested that $M$. aegyptia contained condensed tannin and lignin at low concentrations, making it more suitable for inclusion in ruminant diets.

In another study, M. tridentata (synonym: Xenostegia tridentata) was included in a feeding trial to investigate its use as a supplementary feed to a common foraged grass, Panicum maximum in West African dwarf sheep during the rainy season because of its high acceptability by the animals [45]. In addition, the crude nutrient and tannin contents of $M$. tridentata were analyzed. There was a significant increase in the total food intake in sheep whose ration was supplemented with $M$. tridentata compared with those fed with $P$. maximum only. The crude nutrient analysis revealed higher protein content $(15.3 \% \mathrm{DM})$ in M. tridentata when compared with P. maximum (8.6\% DM). The tannin content $(0.7 \% \mathrm{DM})$ of $M$. tridentata did not reduce its palatability and feeding value [45].

Galat-Luong et al. [46] studied the diet preferences of the Western giant eland group from the native Sudanian habitat to a wildlife reserve in a Sahelian area. Merremia pentaphylla (35.41\% feeding bouts) was the second most preferred food item in the list of herbaceous species consumed by the animal.

In order to develop a suitable feeding strategy for improving grazing sheep production in India, Rajendran and Balakrishnan [47] evaluated the herbage composition, biomass, preference index, and mineral contents in mountain land, fallow land, and waste/roadside land during the Southwest monsoon season. Merremia tridentata and M. emarginata were listed among the herbage species in the mountain land, fallow land, and waste/roadside land. Merremia tridentata had the highest preference index $(2.57 \pm 2.42)$ by the sheep in the waste/roadside land. The maximum preference index recorded in M. tridentata suggests that the species is more edible than other herbage species consumed by the sheep. In addition, the mineral contents analysis revealed that $M$. tridentata and M. emarginata, together with other herbage species contained several minerals such as $\mathrm{Ca}, \mathrm{Fe}, \mathrm{Cu}, \mathrm{Zn}, \mathrm{Mn}$, and Co above the critical level, but the phosphorous level was below the critical level.

Collectively from these data, it is evident that Merremia species are valuable dietary constituents for humans and particularly for livestock feed. With the ever-increasing human population, changes in consumption patterns caused by raise in income and urbanization, as well as the economic growth of many Asian countries, it is likely that the demand for livestock products will double in the next two decades [48,49]. A major challenge in livestock production in many developing countries is the shortage and fluctuating quantity and quality of feed supply all year round. In addition, livestock is majorly fed on roughages that are of low quality such as sugar cane by-products, cereal straws, all of which contain a high amount of ligno-cellulose materials and are deficient in nutrients (protein, minerals, vitamins, and energy) [49]. These challenges call for supplementation of livestock feed that can improve the low-quality roughages, and contribute to increased profitability and productivity of livestock. Many forb species, including those of the genus Merremia, provide high-quality nutrient resources, particularly when there is inadequate 
forage in the perennial grass-based pasture system [6]. The utilization of Merremia species in supplementing livestock feed is therefore encouraged.

\subsubsection{Ethnomedicinal Uses of Merremia Species}

Various species in the genus Merremia have been reported in different parts of the world for the treatment of different diseases. The species $M$. tridentata $(20 \%)$ and $M$. emarginata $(16 \%)$ are the most commonly reported, and India has the highest number of research articles reporting the ethnomedicinal uses of Merremia species. This may be due to India's long history of well-developed traditional medical systems (Ayurveda) [50].

In India, a decoction made from leaves of M. emarginata has been employed in the treatment of fever, neuralgia, urinary infection, rheumatism, liver and kidney diseases [51], inflammation, cough, and headache [21]. The roots of $M$. tridentata prepared by maceration are beneficial in the treatment of diabetes [52] rheumatism, hemiplegia, piles, swellings, and urinary infections [53]. In addition, the whole plant and aerial parts of $M$. tridentata, prepared by maceration have been recorded to be useful in the treatment of leprosy, piles, swellings, rheumatism, stiffness of the joints, hemiplegia, urinary infections [54], and toothache [55].

In Indonesia, the leaf and whole plant of M. mammosa, prepared by infusion and maceration, respectively, have been reported to be beneficial in the treatment and management of diabetes [56] and diabetic ulcers [57]. The leaves of M. peltata prepared by maceration have been employed in the treatment of different forms of cancer, diarrhea, abdominal pain, cough, sore eyes, wounds, and inflammation [58].

The whole plant of M. peltata prepared by maceration has been used as an antiinflammatory, analgesic, anticancer, anti-viral, anti-malarial, anti-bacterial, and anti-fungal in the Philippines [19].

In Malaysia, the leaves [22] and aerial parts [59] of M. borneensis prepared by maceration have been employed in the treatment and relief of breast cancer.

In China, a decoction prepared from the whole plant of M. yunnanensis has been used to treat typhoid and stroke [60]. An infusion from the leaf and fruit of M. yunnanensis is taken to treat stroke, hemiplegia, typhoid fever, and headache [61].

In Columbia, the leaves of M. umbellata prepared by maceration are used as an antibacterial, antifungal, and anti-inflammatory agent [62].

The leaves of M. vitifolia prepared by maceration are used in the treatment of fever, headache, eye inflammation, rheumatism, dysentery, jaundice, and urinary diseases in Bangladesh [20]. The ethnomedicinal uses of the different species in the genus Merremia are summarized in Table 4.

Table 4. Ethnomedicinal uses of Merremia species.

\begin{tabular}{|c|c|c|c|c|c|c|}
\hline Species & $\begin{array}{c}\text { Ethnomedicinal } \\
\text { Uses }\end{array}$ & Part Used & $\begin{array}{c}\text { Type of } \\
\text { Extraction }\end{array}$ & Country & $\begin{array}{l}\text { Location of } \\
\text { Collection }\end{array}$ & Reference \\
\hline \multirow[t]{3}{*}{ M. borneensis } & $\begin{array}{l}\text { Relieve breast } \\
\text { cancer }\end{array}$ & Leaf & Maceration & Malaysia & Unspecified & [22] \\
\hline & Hair treatment & Leaf & Maceration & $\begin{array}{c}\text { Brunei } \\
\text { Darussalam }\end{array}$ & Madang & [63] \\
\hline & $\begin{array}{l}\text { Relieve breast } \\
\text { cancer }\end{array}$ & Aerial parts & Maceration & Malaysia & $\begin{array}{c}\text { University of } \\
\text { Malaysia, } \\
\text { Sabah area }\end{array}$ & [59] \\
\hline M. emarginata & $\begin{array}{c}\text { Rheumatism, } \\
\text { neuralgia, } \\
\text { cough, and } \\
\text { headache }\end{array}$ & Leaf & Maceration & India & $\begin{array}{l}\text { Dharmapuri, } \\
\text { Tamil Nadu }\end{array}$ & {$[21]$} \\
\hline
\end{tabular}


Table 4. Cont.

\begin{tabular}{|c|c|c|c|c|c|c|}
\hline Species & $\begin{array}{c}\text { Ethnomedicinal } \\
\text { Uses }\end{array}$ & Part Used & $\begin{array}{c}\text { Type of } \\
\text { Extraction }\end{array}$ & Country & $\begin{array}{l}\text { Location of } \\
\text { Collection }\end{array}$ & Reference \\
\hline & $\begin{array}{l}\text { Antimicrobial } \\
\text { effect, anti- } \\
\text { inflammatory } \\
\text { activity }\end{array}$ & Leaf & Maceration & India & $\begin{array}{l}\text { Dharmapuri, } \\
\text { Tamil Nadu }\end{array}$ & {$[64]$} \\
\hline & $\begin{array}{c}\text { Fever, } \\
\text { neuralgia, } \\
\text { urinary } \\
\text { infection, } \\
\text { rheumatism, } \\
\text { inflammation, } \\
\text { liver and } \\
\text { kidney diseases }\end{array}$ & Leaf & $\begin{array}{l}\text { Maceration and } \\
\text { decoction }\end{array}$ & India & $\begin{array}{l}\text { Varakkalpattu } \\
\text { village, } \\
\text { Cuddalore } \\
\text { District, Tamil } \\
\text { Nadu }\end{array}$ & [51] \\
\hline \multirow[t]{3}{*}{ M. mammosa } & Breast cancer & Whole plant & Maceration & Indonesia & Surabaya & [65] \\
\hline & $\begin{array}{l}\text { Diabetic } \\
\text { therapy }\end{array}$ & Leaf & Infusion & Indonesia & $\begin{array}{c}\text { Meru Betiri } \\
\text { National Park, } \\
\text { Jember }\end{array}$ & [56] \\
\hline & Diabetic ulcers & Whole plant & Maceration & Indonesia & $\begin{array}{l}\text { Klaten, Central } \\
\text { Java Province }\end{array}$ & [57] \\
\hline \multirow[t]{2}{*}{ M. peltata } & $\begin{array}{c}\text { Anti- } \\
\text { inflammatory, } \\
\text { analgesic, } \\
\text { anti-cancer, } \\
\text { anti-viral, } \\
\text { anti-malarial, } \\
\text { anti-bacterial, } \\
\text { and anti-fungal }\end{array}$ & Whole plant & Maceration & Philippines & $\begin{array}{l}\text { Rogongon, } \\
\text { Iligan City }\end{array}$ & [19] \\
\hline & $\begin{array}{c}\text { Anti-cancer, } \\
\text { diarrhea, } \\
\text { abdominal pain, } \\
\text { cough, sore } \\
\text { eyes, wound } \\
\text { and } \\
\text { inflammation }\end{array}$ & Leaf & $\begin{array}{l}\text { Maceration and } \\
\text { Fractionation }\end{array}$ & Indonesia & $\begin{array}{l}\text { Padang City, } \\
\text { West Sumatra }\end{array}$ & [58] \\
\hline \multirow[t]{2}{*}{ M. tridentata } & $\begin{array}{l}\text { Rheumatism, } \\
\text { hemiplegia, } \\
\text { piles, swellings, } \\
\text { and urinary } \\
\text { disorders }\end{array}$ & Root & Maceration & India & Udupi, Manipal & [53] \\
\hline & Toothache & Whole plant & Maceration & India & $\begin{array}{c}\text { Xavier's } \\
\text { College } \\
\text { campus, } \\
\text { Palayamkottai, } \\
\text { Tirunelveli } \\
\text { District, Tamil } \\
\text { Nadu }\end{array}$ & [55] \\
\hline
\end{tabular}


Table 4. Cont

\begin{tabular}{|c|c|c|c|c|c|c|}
\hline Species & $\begin{array}{c}\text { Ethnomedicinal } \\
\text { Uses }\end{array}$ & Part Used & $\begin{array}{c}\text { Type of } \\
\text { Extraction }\end{array}$ & Country & $\begin{array}{l}\text { Location of } \\
\text { Collection }\end{array}$ & Reference \\
\hline & $\begin{array}{l}\text { Astringent, } \\
\text { calefacient, } \\
\text { laxative, } \\
\text { anodyne, } \\
\text { hemiplegia, } \\
\text { hemorrhoids, } \\
\text { uropathy, } \\
\text { mouth wash, } \\
\text { piles, } \\
\text { inflammation, } \\
\text { fever, and } \\
\text { leprosy }\end{array}$ & Aerial parts & Maceration & India & $\begin{array}{c}\text { Tamil Nadu } \\
\text { Medicinal Plant } \\
\text { Farms and } \\
\text { Herbal } \\
\text { Medicine, } \\
\text { Chennai }\end{array}$ & {$[66]$} \\
\hline & $\begin{array}{l}\text { Piles, swellings, } \\
\text { rheumatism, } \\
\text { stiffness of the } \\
\text { joints, } \\
\text { hemiplegia, and } \\
\text { urinary } \\
\text { infections }\end{array}$ & Whole plant & Maceration & India & $\begin{array}{l}\text { Coimbatore, } \\
\text { Tamil Nadu }\end{array}$ & [54] \\
\hline & $\begin{array}{c}\text { Treatment of } \\
\text { diabetes }\end{array}$ & Root & Maceration & India & $\begin{array}{l}\text { Coimbatore, } \\
\text { Tamil Nadu }\end{array}$ & [52] \\
\hline M. umbellata & $\begin{array}{l}\text { Antibacterial, } \\
\text { antifungal, and } \\
\text { anti- } \\
\text { inflammatory }\end{array}$ & Leaf & Maceration & Colombia & $\begin{array}{l}\text { Pueblo Nuevo, } \\
\text { Bolívar, }\end{array}$ & [60] \\
\hline \multirow[t]{2}{*}{ M. yunnanensis } & $\begin{array}{l}\text { Typhoid and } \\
\text { stroke } \\
\text { treatment }\end{array}$ & Whole plant & Decoction & China & $\begin{array}{c}\text { Heqing } \\
\text { Country, Dali } \\
\text { Prefecture, } \\
\text { Yunnan } \\
\text { province }\end{array}$ & [60] \\
\hline & $\begin{array}{l}\text { Stroke } \\
\text { hemiplegia, } \\
\text { typhoid fever, } \\
\text { and headache }\end{array}$ & Fruit/Leaf & Infusion & China & $\begin{array}{c}\text { Heqing County } \\
\text { of Yunnan } \\
\text { Province }\end{array}$ & [61] \\
\hline M. vitifolia & $\begin{array}{c}\text { Fever, } \\
\text { headache, eye } \\
\text { inflammation, } \\
\text { rheumatism, } \\
\text { dysentery, } \\
\text { jaundice, and } \\
\text { urinary } \\
\text { diseases }\end{array}$ & Leaf & Maceration & Bangladesh & Chittagong & [20] \\
\hline
\end{tabular}

Generally, from the reported ethnomedicinal uses of the plant, the herbal formulations of the species are prepared by decoction, maceration, and infusion (Table 4). Furthermore, the leaves of the different species are the most widely utilized (50\%) part in ethnomedicine, followed by the whole plant $(25.0 \%)$, aerial parts $(12.50 \%)$, roots $(8.33 \%)$, and fruits $(4.16 \%)$.

\subsubsection{Phytochemistry of Species in the Genus Merremia}

A wide array of phytochemicals has been identified and isolated from different extracts and fractions of Merremia species. Phytochemical investigations have been conducted on the whole plant, the aerial parts, and other individual plant parts including roots and leaves. Most of the reported phytochemicals were found in the roots (Table 5a). However, there 
are overlapping compounds obtained from different parts of the plant and across species from the genus. Extraction methods are mainly maceration and infusion. The major class of the phytoconstituents widely isolated and identified from this genus is resin glycosides. Other classes of phytoconstituents from this genus are flavonoids, tropane alkaloids, phenolic compounds, isoflavones, coumarins, and sesquiterpenoid. Many of the compounds isolated in the genus Merremia are therapeutically relevant, with some used as pharmaceutical ingredients in a few commercial drugs.

Devadasu et al. [67] carried out phytochemical screening of M. emarginata leaves. The results revealed the presence of alkaloids, steroids, glycosides, flavonoids, and phenols. In the same study, a prenylflavonoid, 8-prenylnaringenin (1) was isolated from the leaves of M. emarginata using silica thin layer chromatography. In addition to the good antioxidant activity displayed by 8-prenylnaringenin (1) in the study, this compound is a known phytoestrogen and also has anticarcinogen potential [68].

Phytochemical investigations conducted on the root of $M$. yunnanensis for the first time led to the isolation and structure elucidation of two new eudesmane derivatives viz: $1 \alpha, 6 \beta, 9 \beta$-trihydroxy-eudesm-3-ene-1-O- $\beta$-D-glucopyranoside (29) and $1 \alpha, 6 \beta, 9 \beta$-trihydroxyeudesm-3-ene-1-(6-cinnamoyl)-O- $\beta$ - D-glucopyranoside (30) [60]. In another recent study, phytochemical investigations were conducted on M. yunnanensis (root and leaves) [61]. This study led to the isolation of a new compound, namely $1 \alpha, 4 \beta, 8 \beta, 9 \beta$-eudesmane-tetrol$1-O-\beta$-D-glucopyranoside (31), together with the isolation of other known compounds viz: tyrosol (35), hydroxypinoresinol (58), scopoletin (42), hydroxycoumarin (43), quercetin7-O-glucoside (3), and 2-C-methylerythritol (6). The researchers further reported that compounds (6), (43), and (58) were reported for the first time in the genus Merremia, and compounds (31) and (35) were isolated and reported for the first time in the family Convolvulaceae. The results imply that the compounds isolated in M. yunnanensis can be considered important chemotaxonomic markers for M. yunnanensis. Some of the isolated compounds in M. yunnanensis are therapeutically relevant. For example, tyrosol (35) is well known as a strong antioxidant [69]. Antioxidants have health benefits, quenching reactive oxidants [70,71] and slowing down cell damage/death and inflammation. Quercetin-7-Oglucoside (3) has been reported to suppress pancreatic cancer by inhibiting the epidermal growth factor receptor (EGFR) signaling in the cell [72]. Rutin has been reported to inhibit SARS-CoV-2 main protease proteins and could be a potential cure for SARS-CoV-2 [73].

In another study, five new pentasaccharide resin glycosides, named merremins $\mathrm{A}-\mathrm{E}$ (7-10), (13), two new pentasaccharide resin glycoside methyl esters, named merremins F (16) and G (17), and four known resin glycosides, murucoidin IV (12), murucoidin V (14), stoloniferin IV (11), and murucoidin XVII (15) were isolated for the first time from the aerial parts of $M$. hederacea. All these compounds showed multi-drug resistance reversal activities when evaluated further [74].

A chemotaxonomy study of the genus Merremia (using 18 species) based on the distribution of tropane alkaloids revealed a total of 74 tropanes and 13 pyrrolidines based on GC-MS analysis [15]. This study further led to the isolation and structure elucidation of four new aromatic $3 \alpha$-acyloxytropanes named merresectines A-D $(45,47-49)$, (from the roots of $M$. dissecta and M. guerichii), one new $3 \alpha, 6 \beta$-di-(4-methoxybenzoyloxy) tropane (named merredissine (53)) from $M$. dissecta, and datumetine (46) (from M. dissecta and $M$. guerichii). The results from this study led to the grouping of Merremia species into three chemo-taxonomical categories: (1) taxa free of tropanes, (2) taxa with simple tropanes, and (3) taxa with merresectines in addition to simple tropanes. This grouping contributes to the solution of infrageneric taxonomic problems in the genus. According to the study of Rahman et al. [73], caffeic acid derivatives (CAFDs) isolated in M. umbellata [64,75] were reported to inhibit SARS-CoV-2 [76]. Esculetin (44) and luteolin (5) isolated in M. umbellata [75] were reported to decrease angiotensin-converting enzyme 2 (ACE-2) expression, and could therefore reduce SARS-CoV-2 infection [77]. However, rosmarinic acid (37) isolated in M. umbellata [64,75] was reported to increase the expression of ACE-2, which could aggravate SARS-CoV-2 infection [77]. Shimming et al. [14] reported the presence 
of calystegine $B_{2}(55)$ in the leaves and flowers of $M$. dissecta. This compound has been reported to have antidiabetic properties as it was a potent inhibitor of $R$-galactosidases and $\alpha$-glucosidases [78]. Using ultra-performance liquid chromatography-tandem mass spectrometry (UPLC-MS/MS) and reverse phase-high performance liquid chromatography (RP-HPLC), Angappan et al. [79] identified chlorogenic acid (41) in the aqueous leaf extract of $M$. emarginata, which was attributed to the diuretic activity of the extract. In another study, Santos et al. [80] isolated ursolic acid (59) and cis-tiliroside (2) from M. tomentosa leaf extract, and these compounds were thought to be responsible for the insecticidal activity of the extract. The medicinal characteristic of the species of Merremia and other medicinal plants depends on the structure, quantity, and quality of the phytochemical constituents. The phytochemicals detected and isolated in the genus Merremia, alongside their structures are summarized in Table 5, and Figure 4.

Table 5. Phytochemicals isolated from the genus Merremia.

\begin{tabular}{|c|c|c|c|c|c|c|c|}
\hline Species & $\begin{array}{c}\text { Isolated } \\
\text { Compounds }\end{array}$ & $\begin{array}{l}\text { Molecular } \\
\text { Formula }\end{array}$ & $\begin{array}{l}\text { Class of } \\
\text { Isolated } \\
\text { Compound }\end{array}$ & Part & $\begin{array}{c}\text { Extraction } \\
\text { Type }\end{array}$ & $\begin{array}{c}\text { Bioactivity } \\
\text { of the Tested } \\
\text { Isolated } \\
\text { Compound }\end{array}$ & Reference \\
\hline \multirow[t]{2}{*}{ M. emarginata } & $\begin{array}{c}\text { 8- } \\
\text { prenylnaringenin } \\
(\mathbf{1})\end{array}$ & $\mathrm{C}_{20} \mathrm{H}_{20} \mathrm{O}_{5}$ & Flavonoid & Leaf & Maceration & $\begin{array}{l}\text { Antioxidant } \\
\text { and antimy- } \\
\text { cobacterial } \\
\text { activities }\end{array}$ & {$[67]$} \\
\hline & $\begin{array}{l}\text { Chlorogenic acid } \\
\qquad(41)\end{array}$ & $\mathrm{C}_{16} \mathrm{H}_{18} \mathrm{O}_{9}$ & $\begin{array}{l}\text { Phenolic } \\
\text { compound }\end{array}$ & Leaf & Maceration & $\begin{array}{l}\text { Diuretic } \\
\text { activity }\end{array}$ & {$[79]$} \\
\hline \multirow[t]{8}{*}{ M. hederacea } & Merremin A (7) & $\mathrm{C}_{58} \mathrm{H}_{98} \mathrm{O}_{26}$ & $\begin{array}{c}\text { Resin } \\
\text { glycoside }\end{array}$ & Aerial & Infusion & $\begin{array}{l}\text { Multidrug } \\
\text { resistance } \\
\text { reversal } \\
\text { activity }\end{array}$ & {$[74]$} \\
\hline & Merremin B (8) & $\mathrm{C}_{61} \mathrm{H}_{104} \mathrm{O}_{26}$ & $\begin{array}{l}\text { Resin } \\
\text { glycoside }\end{array}$ & Aerial & Infusion & $\begin{array}{l}\text { Multidrug } \\
\text { resistance } \\
\text { reversal } \\
\text { activity }\end{array}$ & {$[74]$} \\
\hline & Merremin C (9) & $\mathrm{C}_{59} \mathrm{H}_{98} \mathrm{O}_{26}$ & $\begin{array}{l}\text { Resin } \\
\text { glycoside }\end{array}$ & Aerial & Infusion & $\begin{array}{l}\text { Multidrug } \\
\text { resistance } \\
\text { reversal } \\
\text { activity }\end{array}$ & {$[74]$} \\
\hline & Merremin D (10) & $\mathrm{C}_{63} \mathrm{H}_{108} \mathrm{O}_{26}$ & $\begin{array}{c}\text { Resin } \\
\text { glycoside }\end{array}$ & Aerial & Infusion & $\begin{array}{l}\text { Multidrug } \\
\text { resistance } \\
\text { reversal } \\
\text { activity }\end{array}$ & {$[74]$} \\
\hline & Murucoidin IV (12) & $\mathrm{C}_{57} \mathrm{H}_{98} \mathrm{O}_{29}$ & $\begin{array}{l}\text { Resin } \\
\text { glycoside }\end{array}$ & Aerial & Infusion & $\begin{array}{l}\text { Multidrug } \\
\text { resistance } \\
\text { reversal } \\
\text { activity }\end{array}$ & {$[74]$} \\
\hline & Stoloniferin IV (11) & $\mathrm{C}_{61} \mathrm{H}_{106} \mathrm{O}_{25}$ & $\begin{array}{c}\text { Resin } \\
\text { glycoside }\end{array}$ & Aerial & Infusion & $\begin{array}{l}\text { Multidrug } \\
\text { resistance } \\
\text { reversal } \\
\text { activity }\end{array}$ & {$[74]$} \\
\hline & Merremin E (13) & $\mathrm{C}_{51} \mathrm{H}_{88} \mathrm{O}_{24}$ & $\begin{array}{c}\text { Resin } \\
\text { glycoside }\end{array}$ & Aerial & Infusion & $\begin{array}{l}\text { Multidrug } \\
\text { resistance } \\
\text { reversal } \\
\text { activity }\end{array}$ & {$[74]$} \\
\hline & Murucoidin V (14) & $\mathrm{C}_{56} \mathrm{H}_{96} \mathrm{O}_{25}$ & $\begin{array}{c}\text { Resin } \\
\text { glycoside }\end{array}$ & Aerial & Infusion & $\begin{array}{l}\text { Multidrug } \\
\text { resistance } \\
\text { reversal } \\
\text { activity }\end{array}$ & {$[74]$} \\
\hline
\end{tabular}


Table 5. Cont.

\begin{tabular}{|c|c|c|c|c|c|c|c|}
\hline Species & $\begin{array}{c}\text { Isolated } \\
\text { Compounds }\end{array}$ & $\begin{array}{l}\text { Molecular } \\
\text { Formula }\end{array}$ & $\begin{array}{l}\text { Class of } \\
\text { Isolated } \\
\text { Compound }\end{array}$ & Part & $\begin{array}{l}\text { Extraction } \\
\text { Type }\end{array}$ & $\begin{array}{c}\text { Bioactivity } \\
\text { of the Tested } \\
\text { Isolated } \\
\text { Compound }\end{array}$ & Reference \\
\hline & $\begin{array}{l}\text { Murucoidin XVII } \\
\qquad(\mathbf{1 5})\end{array}$ & $\mathrm{C}_{65} \mathrm{H}_{101} \mathrm{O}_{26}$ & $\begin{array}{l}\text { Resin } \\
\text { glycoside }\end{array}$ & Aerial & Infusion & $\begin{array}{l}\text { Multidrug } \\
\text { resistance } \\
\text { reversal } \\
\text { activity }\end{array}$ & [74] \\
\hline & Merremin F (16) & $\mathrm{C}_{52} \mathrm{H}_{92} \mathrm{O}_{25}$ & $\begin{array}{l}\text { Resin } \\
\text { glycoside }\end{array}$ & Aerial & Infusion & $\begin{array}{l}\text { Multidrug } \\
\text { resistance } \\
\text { reversal } \\
\text { activity }\end{array}$ & [74] \\
\hline & Merremin G (17) & $\mathrm{C}_{52} \mathrm{H}_{92} \mathrm{O}_{25}$ & $\begin{array}{l}\text { Resin } \\
\text { glycoside }\end{array}$ & Aerial & Infusion & $\begin{array}{l}\text { Multidrug } \\
\text { resistance } \\
\text { reversal } \\
\text { activity }\end{array}$ & {$[74]$} \\
\hline \multirow[t]{2}{*}{ M. tomentosa } & Ursolic acid (59) & $\mathrm{C}_{30} \mathrm{H}_{48} \mathrm{O}_{3}$ & Triterpenoid & Leaf & Maceration & $\begin{array}{l}\text { Insecticidal } \\
\text { activity }\end{array}$ & [80] \\
\hline & cis-Tiliroside (2) & $\mathrm{C}_{30} \mathrm{H}_{26} \mathrm{O}_{13}$ & Flavonoid & Leaf & Maceration & $\begin{array}{l}\text { Insecticidal } \\
\text { activity }\end{array}$ & [80] \\
\hline \multirow[t]{9}{*}{$\begin{array}{c}\text { M. } \\
\text { yunnanensis }\end{array}$} & $\begin{array}{c}1 \alpha, 6 \beta, 9 \beta- \\
\text { trihydroxy- } \\
\text { eudesm-3-ene-1-O- } \\
\beta \text {-D- } \\
\text { glucopyranoside } \\
(\mathbf{2 9})\end{array}$ & $\mathrm{C}_{21} \mathrm{H}_{36} \mathrm{O}_{8}$ & Sesquiterpenoid & Root & Decoction & $\mathrm{N} / \mathrm{A}$ & {$[60]$} \\
\hline & $\begin{array}{c}1 \alpha, 6 \beta, 9 \beta- \\
\text { trihydroxy- } \\
\text { eudesm-3-ene-1-(6- } \\
\text { cinnamoyl)-O- } \beta \text {-D- } \\
\text { glucopyranoside } \\
(\mathbf{3 0})\end{array}$ & $\mathrm{C}_{30} \mathrm{H}_{42} \mathrm{O}_{9}$ & Sesquiterpenoid & Root & Decoction & $\mathrm{N} / \mathrm{A}$ & {$[60]$} \\
\hline & $\begin{array}{c}\text { Eeudesmane- } \\
1 \alpha, 4 \beta, 8 \beta, 9 \beta \\
\text {-tetrol-1-O- } \beta \text { - D } \\
\text {-glucopyranoside } \\
\text { (31) }\end{array}$ & $\mathrm{C}_{21} \mathrm{H}_{38} \mathrm{O}_{9}$ & Sesquiterpenoid & Root & Infusion & $\mathrm{N} / \mathrm{A}$ & {$[61]$} \\
\hline & Tyrosol (35) & $\mathrm{C}_{8} \mathrm{H}_{10} \mathrm{O}_{2}$ & $\begin{array}{l}\text { Phenolic } \\
\text { compound }\end{array}$ & Root & Infusion & $\mathrm{N} / \mathrm{A}$ & [61] \\
\hline & $\begin{array}{c}\text { Hydroxypinoresinol } \\
\text { (58) }\end{array}$ & $\mathrm{C}_{20} \mathrm{H}_{22} \mathrm{O}_{7}$ & Lignan & Leaf & Infusion & $\mathrm{N} / \mathrm{A}$ & {$[61]$} \\
\hline & Scopoletin (42) & $\mathrm{C}_{10} \mathrm{H}_{8} \mathrm{O}_{4}$ & Coumarin & Leaf & Infusion & $\mathrm{N} / \mathrm{A}$ & {$[61]$} \\
\hline & $\begin{array}{l}\text { Hydroxycoumarin } \\
\text { (43) }\end{array}$ & $\mathrm{C}_{9} \mathrm{H}_{6} \mathrm{O}_{3}$ & Coumarin & Leaf & Infusion & $\mathrm{N} / \mathrm{A}$ & [61] \\
\hline & $\begin{array}{l}\text { Quercetin-7-O- } \\
\text { glucoside } \\
\text { (3) }\end{array}$ & $\mathrm{C}_{21} \mathrm{H}_{20} \mathrm{O}_{12}$ & Flavonoid & Root & Infusion & $\mathrm{N} / \mathrm{A}$ & [61] \\
\hline & $\begin{array}{c}2-C- \\
\text { methylerythritol } \\
(6)\end{array}$ & $\mathrm{C}_{5} \mathrm{H}_{12} \mathrm{O}_{4}$ & Polyol & Leaf & infusion & $\mathrm{N} / \mathrm{A}$ & {$[61]$} \\
\hline
\end{tabular}


Table 5. Cont.

\begin{tabular}{|c|c|c|c|c|c|c|c|}
\hline Species & $\begin{array}{c}\text { Isolated } \\
\text { Compounds }\end{array}$ & $\begin{array}{l}\text { Molecular } \\
\text { Formula }\end{array}$ & $\begin{array}{l}\text { Class of } \\
\text { Isolated } \\
\text { Compound }\end{array}$ & Part & $\begin{array}{l}\text { Extraction } \\
\text { Type }\end{array}$ & $\begin{array}{l}\text { Bioactivity } \\
\text { of the Tested } \\
\text { Isolated } \\
\text { Compound }\end{array}$ & Reference \\
\hline \multirow[t]{8}{*}{ M. umbellata } & $\begin{array}{c}\text { SA 2-O- } \beta \text { - D } \\
\text {-( }\left(^{\prime}, 6^{\prime} \text {-dicaffeoyl)- }\right. \\
\text { glucopyranoside } \\
(36)\end{array}$ & $\mathrm{C}_{31} \mathrm{H}_{28} \mathrm{O}_{14}$ & $\begin{array}{l}\text { Phenolic } \\
\text { Compound }\end{array}$ & Whole plant & Maceration & $\begin{array}{l}\text { Allelopathic } \\
\text { effect on } \\
\text { Arabidopsis } \\
\text { seed } \\
\text { germination }\end{array}$ & [75] \\
\hline & $\begin{array}{c}\text { Rosmarinic acid } \\
\text { (37) }\end{array}$ & $\mathrm{C}_{18} \mathrm{H}_{16} \mathrm{O}_{8}$ & $\begin{array}{l}\text { Phenolic } \\
\text { Compound }\end{array}$ & Whole plant & Maceration & $\begin{array}{l}\text { Allelopathic } \\
\text { effect on } \\
\text { Arabidopsis } \\
\text { seed } \\
\text { germination }\end{array}$ & {$[64,75]$} \\
\hline & Paprazine (38) & $\mathrm{C}_{17} \mathrm{H}_{17} \mathrm{NO}_{3}$ & $\begin{array}{l}\text { Phenolic } \\
\text { Compound }\end{array}$ & Whole plant & Maceration & $\begin{array}{l}\text { Allelopathic } \\
\text { effect on } \\
\text { Arabidopsis } \\
\text { seed } \\
\text { germination }\end{array}$ & [75] \\
\hline & $\begin{array}{c}N \text { - } p \text {-cis- } \\
\text { coumaroyltyramine } \\
(39)\end{array}$ & $\mathrm{C}_{17} \mathrm{H}_{17} \mathrm{NO}$ & $\begin{array}{l}\text { Phenolic } \\
\text { Compound }\end{array}$ & Whole plant & Maceration & $\begin{array}{l}\text { Allelopathic } \\
\text { effect on } \\
\text { Arabidopsis } \\
\text { seed } \\
\text { germination }\end{array}$ & [75] \\
\hline & Caffeic acid (40) & $\mathrm{C}_{9} \mathrm{H}_{8} \mathrm{O}_{4}$ & $\begin{array}{l}\text { Phenolic } \\
\text { Compound }\end{array}$ & Whole plant & Maceration & $\begin{array}{l}\text { Allelopathic } \\
\text { effect on } \\
\text { Arabidopsis } \\
\text { seed } \\
\text { germination }\end{array}$ & {$[64,75]$} \\
\hline & Esculetin (44) & $\mathrm{C}_{9} \mathrm{H}_{6} \mathrm{O}_{4}$ & Coumarin & Whole plant & Maceration & $\begin{array}{l}\text { Allelopathic } \\
\text { effect on } \\
\text { Arabidopsis } \\
\text { seed } \\
\text { germination }\end{array}$ & [75] \\
\hline & Quercetin (4) & $\mathrm{C}_{15} \mathrm{H}_{10} \mathrm{O}_{7}$ & Flavonoid & Whole plant & Maceration & $\begin{array}{l}\text { Allelopathic } \\
\text { effect on } \\
\text { Arabidopsis } \\
\text { seed } \\
\text { germination }\end{array}$ & {$[64,75]$} \\
\hline & Luteolin (5) & $\mathrm{C}_{15} \mathrm{H}_{10} \mathrm{O}_{6}$ & Flavonoid & Whole plant & Maceration & $\begin{array}{l}\text { Allelopathic } \\
\text { effect on } \\
\text { Arabidopsis } \\
\text { seed } \\
\text { germination }\end{array}$ & [75] \\
\hline \multirow[t]{3}{*}{$\begin{array}{c}\text { M. } \\
\text { kentrocaulos }\end{array}$} & $\begin{array}{l}\text { Merrekentrones } \\
\text { A and B (32) }\end{array}$ & $\mathrm{C}_{15} \mathrm{H}_{14} \mathrm{O}_{3}$ & Sesquiterpenoid & Root & Maceration & $\mathrm{N} / \mathrm{A}$ & [81] \\
\hline & $\begin{array}{c}\text { Merrekentrones C } \\
\text { (33) }\end{array}$ & $\mathrm{C}_{15} \mathrm{H}_{16} \mathrm{O}_{4}$ & Sesquiterpenoid & Root & Maceration & $\mathrm{N} / \mathrm{A}$ & [81] \\
\hline & $\begin{array}{c}\text { Merrekentrones D } \\
\text { (34) }\end{array}$ & $\mathrm{C}_{15} \mathrm{H}_{18} \mathrm{O}_{3}$ & Sesquiterpenoid & Root & Maceration & $\mathrm{N} / \mathrm{A}$ & [81] \\
\hline M. mammosa & $\begin{array}{c}\text { Mammoside A (22) } \\
\text { and B (23) }\end{array}$ & $\mathrm{C}_{48} \mathrm{H}_{82} \mathrm{O}_{20}$ & $\begin{array}{c}\text { Resin } \\
\text { glycosides }\end{array}$ & Root & Maceration & $\mathrm{N} / \mathrm{A}$ & [82] \\
\hline
\end{tabular}


Table 5. Cont

\begin{tabular}{|c|c|c|c|c|c|c|c|}
\hline Species & $\begin{array}{c}\text { Isolated } \\
\text { Compounds }\end{array}$ & $\begin{array}{l}\text { Molecular } \\
\text { Formula }\end{array}$ & $\begin{array}{l}\text { Class of } \\
\text { Isolated } \\
\text { Compound }\end{array}$ & Part & $\begin{array}{l}\text { Extraction } \\
\text { Type }\end{array}$ & $\begin{array}{c}\text { Bioactivity } \\
\text { of the Tested } \\
\text { Isolated } \\
\text { Compound }\end{array}$ & Reference \\
\hline & $\begin{array}{l}\text { Mammoside } \mathrm{H} 1 \\
(\mathbf{2 0})\end{array}$ & $\mathrm{C}_{54} \mathrm{H}_{92} \mathrm{O}_{25}$ & $\begin{array}{c}\text { Resin } \\
\text { glycosides }\end{array}$ & Root & Maceration & $\mathrm{N} / \mathrm{A}$ & [82] \\
\hline & $\begin{array}{c}\text { Mammoside } \mathrm{H} 2 \\
(\mathbf{2 1})\end{array}$ & $\mathrm{C}_{54} \mathrm{H}_{92} \mathrm{O}_{25}$ & $\begin{array}{c}\text { Resin } \\
\text { glycosides }\end{array}$ & Root & Maceration & $\mathrm{N} / \mathrm{A}$ & [82] \\
\hline $\begin{array}{c}\text { M. } \\
\text { quinquefolia }\end{array}$ & $\begin{array}{l}\text { Merresectine } \\
\text { A (45) }\end{array}$ & $\mathrm{C}_{15} \mathrm{H}_{19} \mathrm{NO}_{3}$ & $\begin{array}{l}\text { Tropane } \\
\text { alkaloid }\end{array}$ & Root & Maceration & $\mathrm{N} / \mathrm{A}$ & [15] \\
\hline $\begin{array}{l}\text { M. cissoides } \\
\text { and M. } \\
\text { quinquefolia }\end{array}$ & $\begin{array}{c}\text { Merresectine } \\
\text { B/kurameric acid } \\
\text { (47) }\end{array}$ & $\mathrm{C}_{31} \mathrm{H}_{45} \mathrm{NO}_{8}$ & $\begin{array}{l}\text { Tropane } \\
\text { alkaloid }\end{array}$ & Root & Maceration & $\mathrm{N} / \mathrm{A}$ & [15] \\
\hline $\begin{array}{l}\text { M. cissoides } \\
\text { and } M . \\
\text { quinquefolia }\end{array}$ & $\begin{array}{c}\text { Merresectine C } \\
\text { (48) }\end{array}$ & $\mathrm{C}_{25} \mathrm{H}_{35} \mathrm{NO}_{3}$ & $\begin{array}{l}\text { Tropane } \\
\text { alkaloid }\end{array}$ & Root & Maceration & $\mathrm{N} / \mathrm{A}$ & [15] \\
\hline $\begin{array}{l}\text { M. cissoides } \\
\text { and } M . \\
\text { quinquefolia }\end{array}$ & Merredissine (53) & $\mathrm{C}_{24} \mathrm{H}_{27} \mathrm{NO}_{6}$ & $\begin{array}{l}\text { Tropane } \\
\text { alkaloid }\end{array}$ & Root & Maceration & $\mathrm{N} / \mathrm{A}$ & [15] \\
\hline M. dissecta & Datumetine (46) & $\mathrm{C}_{16} \mathrm{H}_{21} \mathrm{NO}_{3}$ & $\begin{array}{l}\text { Tropane } \\
\text { alkaloid }\end{array}$ & Root & Maceration & NA & [15] \\
\hline \multirow[t]{2}{*}{ M. guerichii } & $\begin{array}{l}\text { Merresectine D } \\
\qquad(49)\end{array}$ & $\mathrm{C}_{27} \mathrm{H}_{39} \mathrm{NO}_{9}$ & $\begin{array}{l}\text { Tropane } \\
\text { alkaloid }\end{array}$ & Root & Maceration & $\mathrm{N} / \mathrm{A}$ & [15] \\
\hline & $\begin{array}{c}\text { Merresectine } \\
\beta \text {-D-glucoside (50) }\end{array}$ & $\mathrm{C}_{27} \mathrm{H}_{39} \mathrm{NO}_{9}$ & $\begin{array}{l}\text { Tropane } \\
\text { alkaloid }\end{array}$ & Root & Maceration & $\mathrm{N} / \mathrm{A}$ & [15] \\
\hline M. quinata & Consabatine (54) & $\mathrm{C}_{20} \mathrm{H}_{31} \mathrm{NO}_{4}$ & $\begin{array}{l}\text { Tropane } \\
\text { alkaloid }\end{array}$ & Root & Maceration & $\mathrm{N} / \mathrm{A}$ & [15] \\
\hline $\begin{array}{l}\text { M. cissoides } \\
\text { and } M . \\
\text { quinquefolia }\end{array}$ & $\begin{array}{l}\text { Merresectine E (51) } \\
\text { and Merresectine E } \\
\beta \text {-D-glucoside (52) }\end{array}$ & $\mathrm{C}_{20} \mathrm{H}_{27} \mathrm{NO}_{3}$ & $\begin{array}{l}\text { Tropane } \\
\text { alkaloid }\end{array}$ & Root & Maceration & $\mathrm{N} / \mathrm{A}$ & [15] \\
\hline \multirow[t]{2}{*}{ M. tuberosa } & $\begin{array}{l}\text { Octadecanonyl } \\
\text { caffeate (56) }\end{array}$ & $\mathrm{C}_{27} \mathrm{H}_{44} \mathrm{O}_{4}$ & Caffeate ester & Root & Maceration & NA & [83] \\
\hline & $\begin{array}{l}\text { 6- } \\
\text { Methylheptadecanoyl } \\
\text { caffeate (57) }\end{array}$ & $\mathrm{C}_{27} \mathrm{H}_{42} \mathrm{O}_{5}$ & Caffeate ester & Root & Maceration & NA & [83] \\
\hline \multirow[t]{3}{*}{ M. dissecta } & $\begin{array}{l}\text { Octadecanonyl } \\
\text { caffeate (56) }\end{array}$ & $\mathrm{C}_{27} \mathrm{H}_{44} \mathrm{O}_{4}$ & Caffeate ester & Root & Maceration & NA & [83] \\
\hline & $\begin{array}{l}\text { 6- } \\
\text { Methylheptadecanoyl } \\
\text { Caffeate (57) }\end{array}$ & $\mathrm{C}_{27} \mathrm{H}_{42} \mathrm{O}_{5}$ & Caffeate ester & Root & Maceration & NA & [83] \\
\hline & Calystegine $B_{2}(55)$ & $\mathrm{C}_{7} \mathrm{H}_{13} \mathrm{NO}_{4}$ & $\begin{array}{l}\text { Tropane } \\
\text { alkaloid }\end{array}$ & Leaf, Flower & $\mathrm{N} / \mathrm{A}$ & $\begin{array}{l}\text { Antidiabetic } \\
\text { activity }\end{array}$ & {$[14,78]$} \\
\hline \multirow[t]{2}{*}{ M. mammosa } & $\begin{array}{l}\text { Merremoside } \\
\text { A (22) }\end{array}$ & $\mathrm{C}_{50} \mathrm{H}_{86} \mathrm{O}_{20}$ & $\begin{array}{c}\text { Resin } \\
\text { glycosides }\end{array}$ & Tuber & Maceration & NA & [84] \\
\hline & $\begin{array}{l}\text { Merremoside B } \\
\text { (23) }\end{array}$ & $\mathrm{C}_{48} \mathrm{H}_{82} \mathrm{O}_{20}$ & $\begin{array}{c}\text { Resin } \\
\text { glycosides }\end{array}$ & Tuber & Maceration & NA & [84] \\
\hline
\end{tabular}


Table 5. Cont

\begin{tabular}{|c|c|c|c|c|c|c|c|}
\hline Species & $\begin{array}{c}\text { Isolated } \\
\text { Compounds }\end{array}$ & $\begin{array}{l}\text { Molecular } \\
\text { Formula }\end{array}$ & $\begin{array}{l}\text { Class of } \\
\text { Isolated } \\
\text { Compound }\end{array}$ & Part & $\begin{array}{l}\text { Extraction } \\
\text { Type }\end{array}$ & $\begin{array}{l}\text { Bioactivity } \\
\text { of the Tested } \\
\text { Isolated } \\
\text { Compound }\end{array}$ & Reference \\
\hline & $\begin{array}{l}\text { Merremoside C } \\
\text { (24) }\end{array}$ & $\mathrm{C}_{49} \mathrm{H}_{84} \mathrm{O}_{20}$ & $\begin{array}{c}\text { Resin } \\
\text { glycosides }\end{array}$ & Tuber & Maceration & NA & [84] \\
\hline & $\begin{array}{l}\text { Merremoside D } \\
(\mathbf{2 5})\end{array}$ & $\mathrm{C}_{48} \mathrm{H}_{82} \mathrm{O}_{20}$ & $\begin{array}{c}\text { Resin } \\
\text { glycosides }\end{array}$ & Tuber & Maceration & NA & [84] \\
\hline & $\begin{array}{l}\text { Merremoside E } \\
\quad(\mathbf{2 6})\end{array}$ & $\mathrm{C}_{48} \mathrm{H}_{82} \mathrm{O}_{20}$ & $\begin{array}{c}\text { Resin } \\
\text { glycosides }\end{array}$ & Tuber & Maceration & NA & [84] \\
\hline & $\begin{array}{l}\text { Merremoside F } \\
\quad(27)\end{array}$ & $\mathrm{C}_{55} \mathrm{H}_{94} \mathrm{O}_{25}$ & $\begin{array}{c}\text { Resin } \\
\text { glycosides }\end{array}$ & Tuber & Maceration & NA & [85] \\
\hline & $\begin{array}{l}\text { Merremoside } G \\
\quad(\mathbf{2 8})\end{array}$ & $\mathrm{C}_{54} \mathrm{H}_{92} \mathrm{O}_{25}$ & $\begin{array}{c}\text { Resin } \\
\text { glycosides }\end{array}$ & Tuber & Maceration & NA & [85] \\
\hline
\end{tabular}

N/A: Not available.
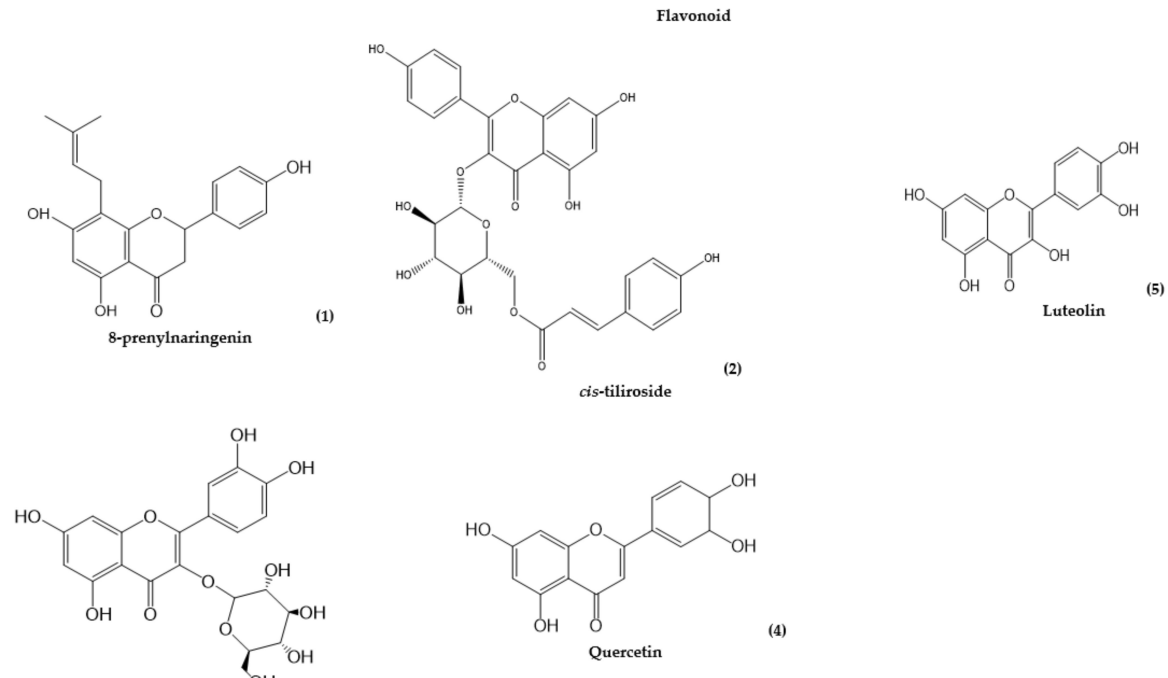

Quercetin-7-O-glucoside (3)

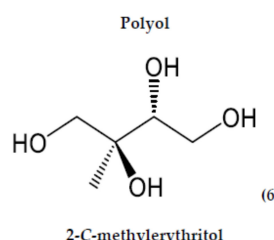

Resin glycoside
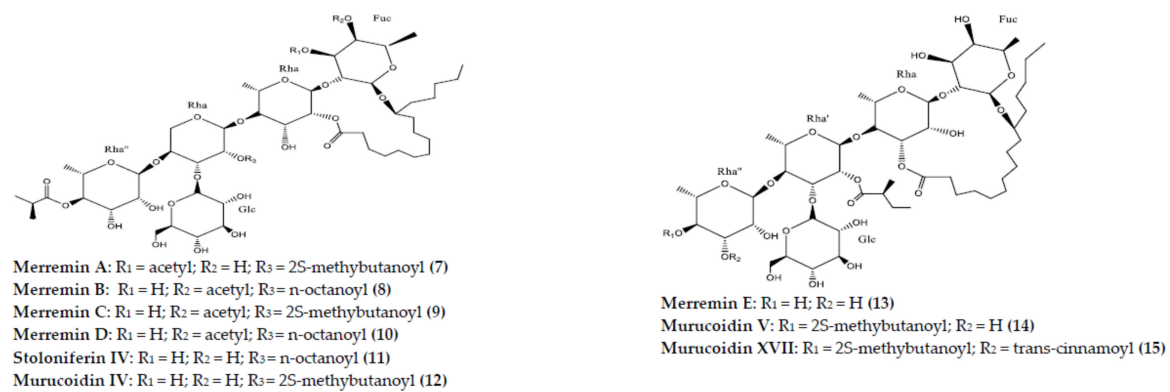

Figure 4. Cont. 


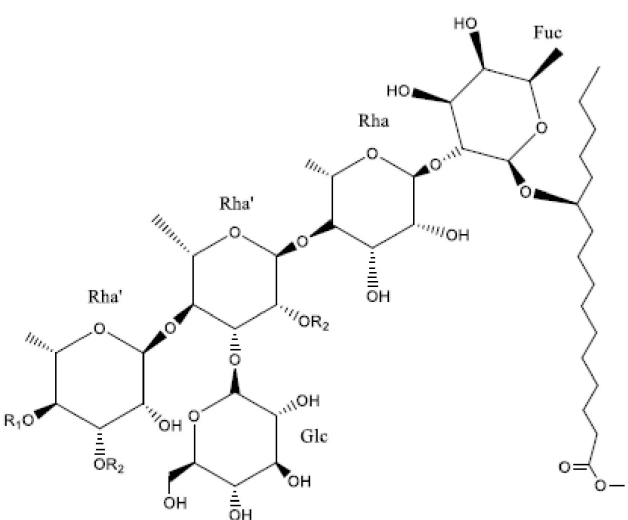

Merremin F: $\mathrm{R}_{1}=2 \mathrm{~S}$-methybutanoyl $\mathrm{R}_{2}=\mathrm{H}$ (16) Merremin G: $\mathrm{R}_{1}=\mathrm{H} ; \mathrm{R}_{2}=$ 2S-methybutanoyl (17)

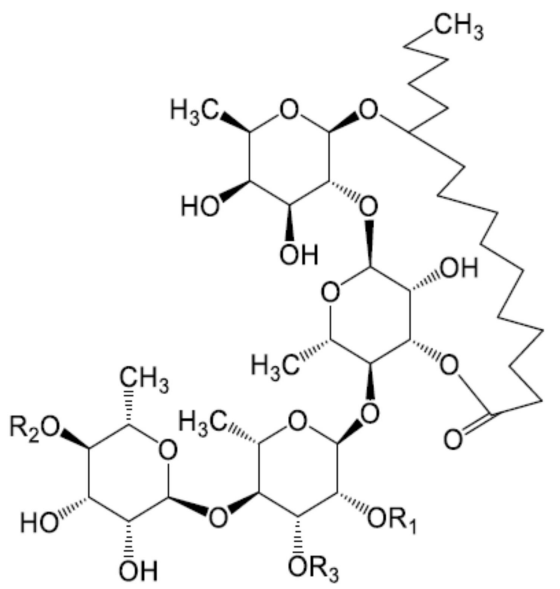

Mammoside $\mathrm{H}_{1}: \mathrm{R}_{1}=\mathrm{COCH}\left(\mathrm{CH}_{3}\right) \mathrm{C}_{2} \mathrm{H}_{5} ; \mathrm{R}_{2}=\mathrm{COCH}\left(\mathrm{CH}_{3}\right)_{2}$ (20) Mammoside $\mathrm{H}_{2}: \mathrm{R} 1=\mathrm{R}_{2} ; \mathrm{R}_{3}=$ Glucose (21)

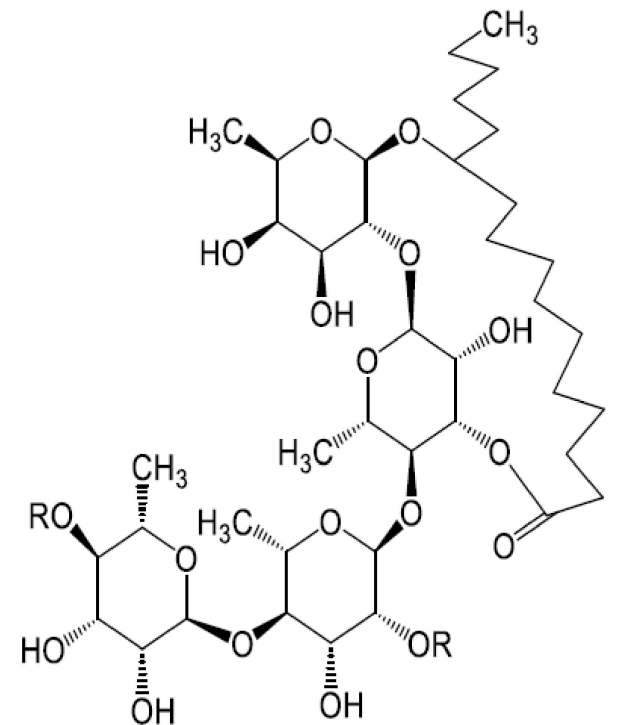

Mammoside A: $\mathrm{R}=\mathrm{COCH}\left(\mathrm{CH}_{3}\right) \mathrm{CH}_{2} \mathrm{H}_{5}$ (18) Mammoside B: $\mathrm{R}=\mathrm{COCH}\left(\mathrm{CH}_{3}\right)_{2}$ (19)
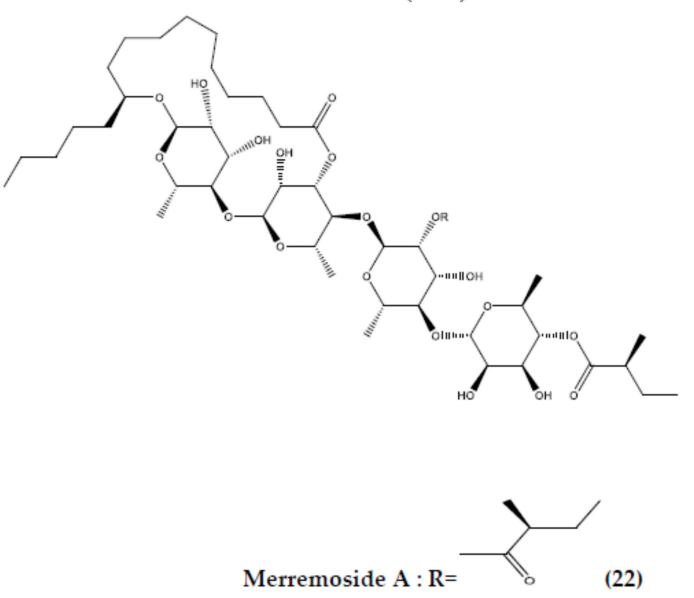

Merremoside $\mathbf{B}: \mathrm{R}=$

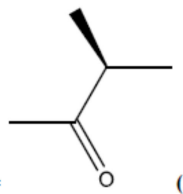

(23)

Figure 4. Cont. 

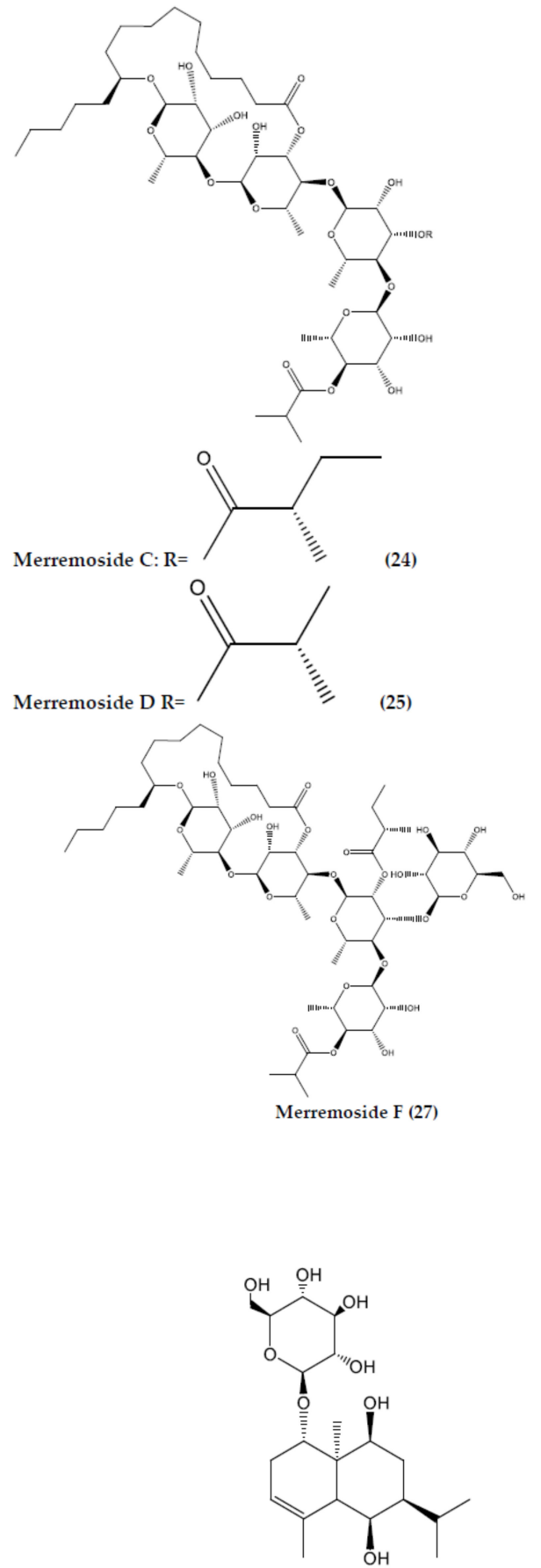

$1 \alpha, 6 \beta, 9 \beta$-trihydroxy-eudesm-3-ene-1-O- $\beta$ - D-glucopyranoside (29)

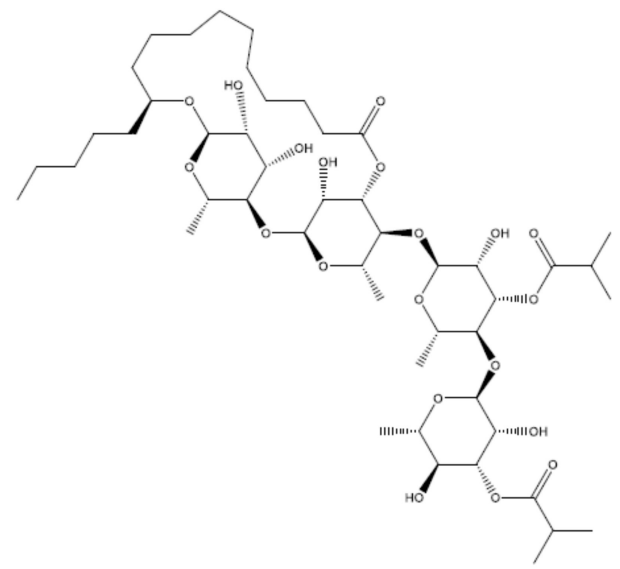

Merremoside E (26)

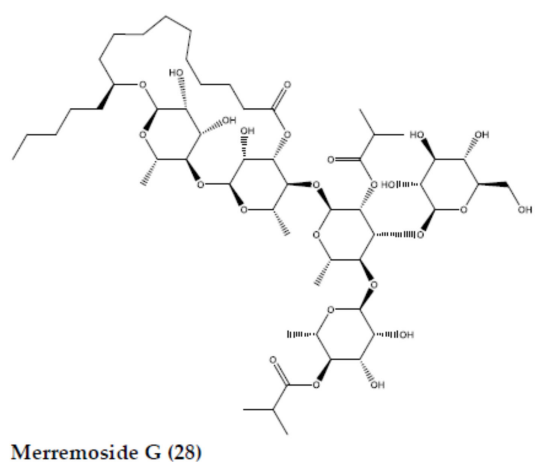

Sesquiterpenoid

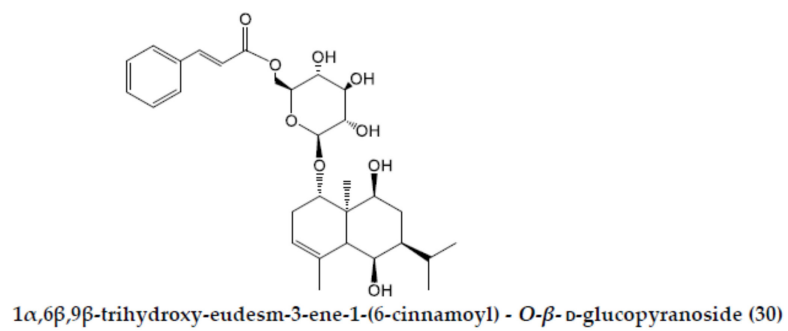

Figure 4. Cont. 


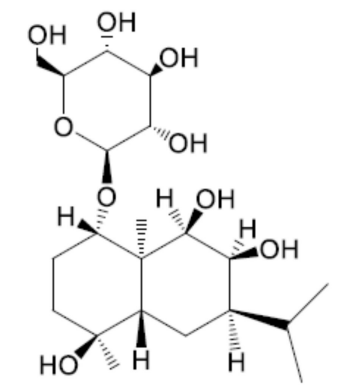

Eeudesmane-1 $\alpha, 4 \beta, 8 \beta, 9 \beta$-tetrol-1-O- $\beta$ - D- glucopyranoside (31)
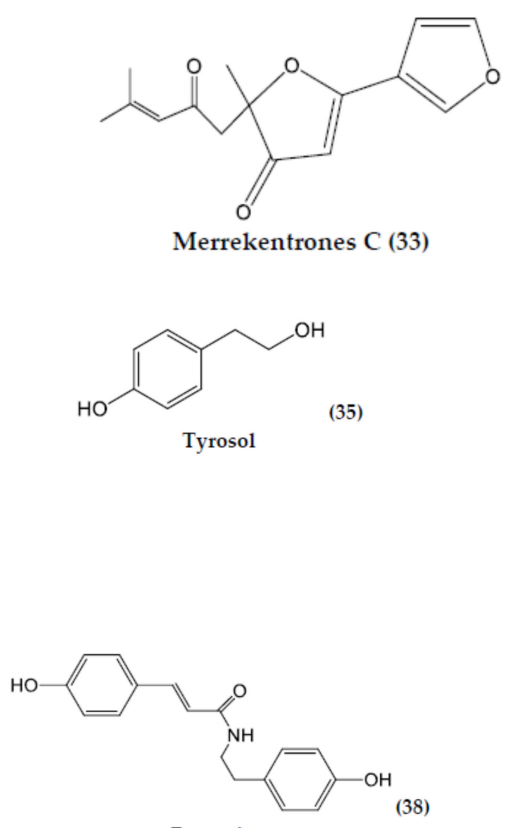

Paprazine

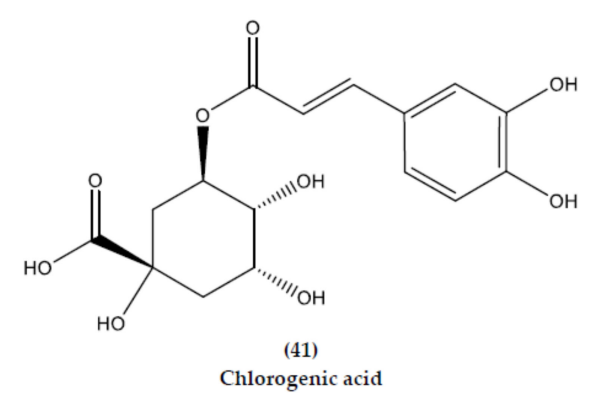

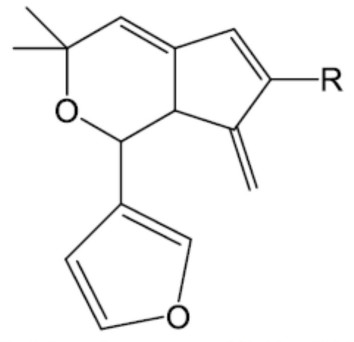

Merrekentrones A: $\mathrm{R}=\mathrm{CH}_{3}$; Merrekentrones (B): $\mathrm{R}=\mathrm{CH}_{2} \mathrm{OH}$ (32)

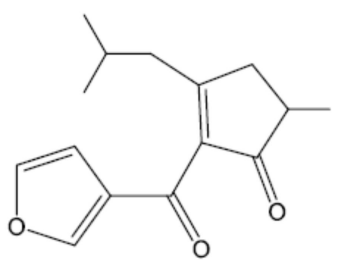

Merrekentrones D (34)

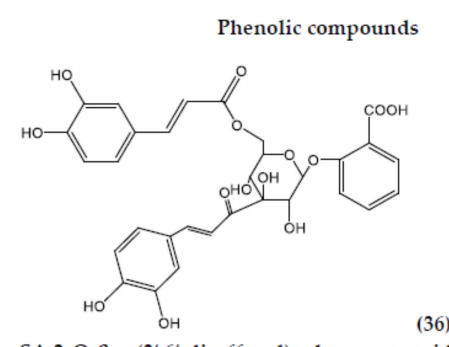

SA 2-O- $\beta$ - D-(3', $6^{\prime}$-dicaffeoyl)- glucopyranoside

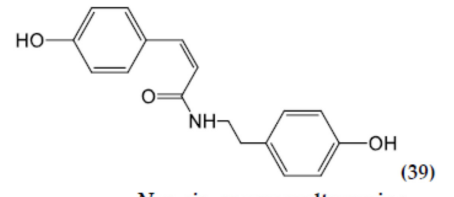

N-p-cis- coumaroyltyramine

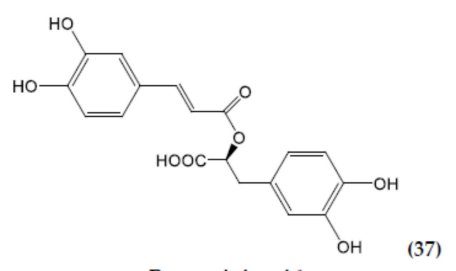

Rosmarinic acid

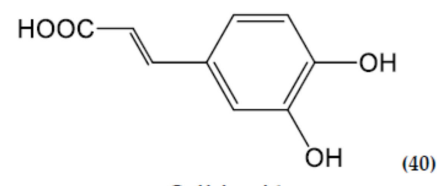

Caffeic acid

Figure 4. Cont. 
<smiles>COc1cc2ccc(=O)oc2cc1O</smiles><smiles>[R7]c1ccc(C(=O)OC2CC3CCC(C2)N3CC)cc1[R]</smiles>

Merresectine A: $\mathrm{R}_{1}=\mathrm{H}, \mathrm{R}_{2}=\mathrm{OCH}_{3}, \mathrm{R}_{3}=\mathrm{H}(45)$ Datumetine $\mathrm{R}_{1}=\mathrm{CH}_{3}, \mathrm{R}_{2}=\mathrm{OCH}_{3}, \mathrm{R}_{3}=\mathrm{H}$ (46)

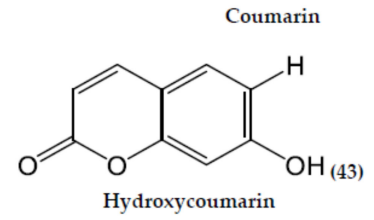

Tropane alkaloid<smiles>[R2]c1c(CC=C(C)C)cc(C(=O)OC2CC3CCC(C2)N3C)cc1CC=C(C)C</smiles>

Merresectine $\mathrm{B} /$ kurameric acid: $\mathrm{R}=\boldsymbol{\beta}$ - D-glucose (47) Merresectine $\mathrm{C}: \mathrm{R}=\mathrm{H}$ (48)
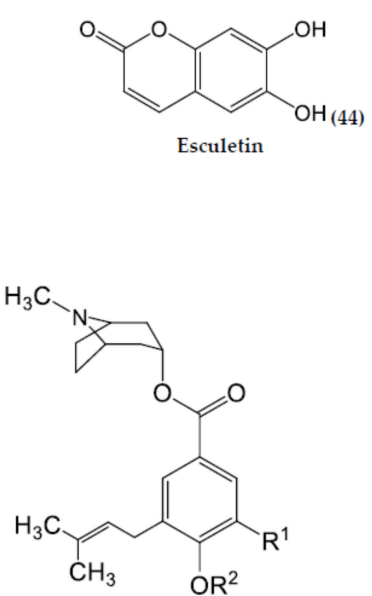

Merresectine D: $\mathrm{R}_{1}=\mathrm{OCH}_{3}, \mathrm{R}_{2}=\mathrm{H}(49)$

Merresectine $\beta$ - D-glucoside: $\mathrm{R}_{1}=\mathrm{OCH}_{3}, \mathrm{R}_{2}=\beta$ - -glucose (50)

Merresectine $E: R_{1}=H, R_{2}=H(51)$

Merresectine $E \beta$ - D-glucose: $R_{1}=H, R_{2}=\beta$ - $D$ - glucose

(52)<smiles>COc1ccc(C(=O)OC2CC3CCC(C2)N3C)cc1</smiles>

(53)

Merredissine<smiles>CC(C)=CCC1=CC(O)(C(=O)OC2CC3CCC(C2)N3C)CC=C1O</smiles>

Consabatine

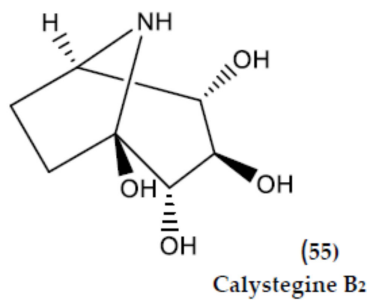

Caffeate ester<smiles>CCCCOC(=O)/C=C/c1ccc(O)c(O)c1</smiles>

(56)<smiles>CCCCCCCCCCCCCCCCCCCC=Cc1ccc(O)c(O)c1</smiles>

6-Methylheptadecanoyl caffeate

Figure 4. Cont. 


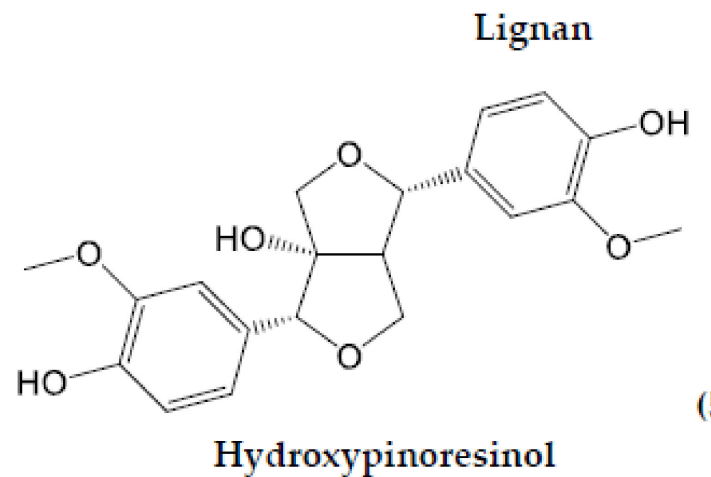

(58)

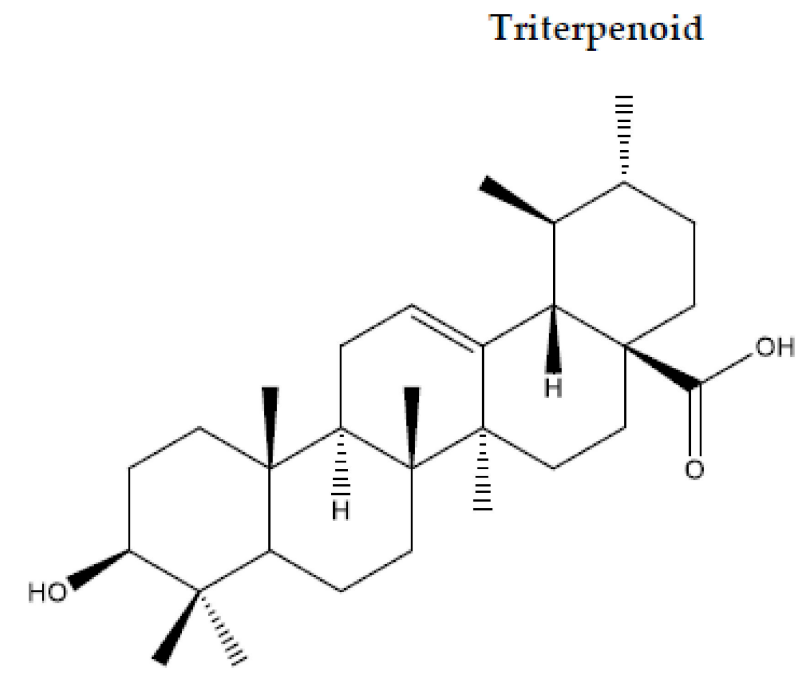

(59)

Ursolic acid

Figure 4. Structures of isolated phytochemicals in the genus Merremia.

\subsubsection{Biological and Pharmacological Activities}

Due to the different ethnomedicinal usage and phytochemicals present in the genus Merremia, several biological and pharmacological have been assessed in 11 species (viz. M. aegyptia, $M$. borneensis, $M$. dissecta, $M$. emarginata, $M$. hederacea, $M$. mammosa, $M$. peltata, M. tridentata, M. tomentosa, M. umbellata, and M. vitifolia). Antimicrobial and antioxidant activities are the most studied, with M. emarginata being the most researched species. The various scientific studies documenting the relevant biological activity of Merremia species are presented below and summarized in Table 6. The activities are presented in terms of the plant extract types, experimental methodologies, extract concentrations/doses, and possible mechanisms of action where the information was available.

\section{Cancer Cell Cytotoxicity}

In recent years, research on medicinal plants as potential chemotherapeutic agents has increased globally because of their actions that prevent cancer initiation and proliferation with limited toxicity, as well as their anti-multidrug reversal ability $[86,87]$. There are very few scientific studies on Merremia species as anticancer agents, but their traditional use in treating breast cancer may have prompted investigations on their potential anticancer and antiproliferative properties as discussed below. The antiproliferative activities of three solvent extracts ( $50 \mu \mathrm{g} \mathrm{mL}^{-1}$ ethyl acetate, hexane, and methanol) from whole plants of $M$. emarginata were investigated on human cancer cell lines using the 3-(4,5-Dimethylthiazol-2yl)-2,5-diphenyltetrazolium bromide (MTT) reduction assay [3]. The results demonstrated antiproliferative effects of the extracts against the cell lines of which the ethyl acetate extract 
was the most effective, inhibiting the proliferation of cell lines A549, KB, MIA-PaCa-2, and DU-145 with $\mathrm{IC}_{50}$ values of $28.5 \mu \mathrm{g} \mathrm{mL}^{-1}, 37.2 \mu \mathrm{g} \mathrm{mL}^{-1}, 51 \mu \mathrm{g} \mathrm{mL}^{-1}$, and $69.4 \mu \mathrm{g} \mathrm{mL}^{-1}$, respectively at $50 \mu \mathrm{g} \mathrm{mL}^{-1}$. In another study, the antiproliferative potential of the leaf extracts (methanol, ethyl acetate, and hexane) of M. emarginata and different parts of nine other medicinal plants on human cancer cell lines and monkey normal kidney epithelial cells (VERO) was investigated [88]. The leaf extracts were tested at different concentrations (range: $3.13-200 \mu \mathrm{g} \mathrm{mL}^{-1}$ ) and time intervals $(24,48$, and $72 \mathrm{~h}$ ). The antiproliferative study was done using the MTT assay, while flow cytometry was used to determine apoptosis. The results revealed that $M$. emarginata hexane extract exhibited cytotoxic effects in all the cell lines in a concentration- and time-dependent manner, ranking fifth out of the 10 plants used in the trial. The hexane extract $\left(25 \mu \mathrm{g} \mathrm{mL}^{-1}\right)$ particularly inhibited A549 and COLO $320 \mathrm{DM}$ cell line proliferation (with $\mathrm{IC}_{50}$ values of $15.5 \mu \mathrm{g} \mathrm{mL}^{-1}$ and $18.4 \mu \mathrm{g}$ $\mathrm{mL}^{-1}$, respectively) and had minimal toxicity $\left(\mathrm{IC}_{50}\right.$ value of $65.2 \mu \mathrm{g} \mathrm{mL} \mathrm{L}^{-1}$ ) for VERO cells at $72 \mathrm{~h}$. The authors speculated that the inhibition of cell line proliferation by $M$. emarginata extract might be due to its antiproliferative potential to demonstrate cancer cell-specific death. Wang et al. [74] tested the cytotoxicity and multidrug resistance reversal activity of different types of isolated pentasaccharide resin glycoside compounds ( $25 \mu \mathrm{M}$ of merremins $A-G(\mathbf{7}-10,13,16,17)$ and murucoidin IV (12), murucoidin V (14), stoloniferin IV (11), and murucoidin XVII (15)) from $M$. hederacea aerial parts in KB/VCR cell lines using a sulforhodamine B assay. Vinblastine served as a reference. Merremins A (7), E (13), $\mathrm{G}$ (17), and murucoidin V (14) were non-cytotoxic at $25 \mathrm{Mm}$ but enhanced the cytotoxicity of vinblastine 2.3-142.5-fold at $25 \mathrm{Mm}$, and demonstrated anti-multidrug reversal activity. Taken together, the results suggest that the investigated extracts and compounds from Merremia species have the potential to be developed into chemotherapeutic agents as they showed significant cytotoxic effects and inhibited the proliferation of cancer cells. However, more in vivo studies and clinical trials are required. Other Merremia species must be investigated for their anticancer activities, particularly those species (M. borneensis, M. peltata and M. mammosa) that have been reported to be used in treating cancer in traditional medicine.

\section{Anti-Diabetic Activity}

Diabetes is a chronic disease marked by high blood sugar resulting from a decrease in insulin production by pancreatic beta cells, or when the body cannot effectively use the insulin it produces $[89,90]$. In 2019, diabetes was the direct cause of 1.5 million deaths globally, and it has been projected that diabetes will be the seventh leading cause of death by 2030 [89]. Additionally, diabetes has been associated with increased mortality and severity of COVID-19 disease [91,92]. Several alpha-glucosidase inhibitor drugs (such as Miglitor Acarbose, and Voglibose) are used in the treatment of diabetes [90]. Several in vivo and in vitro studies have reported that members of the genus Merremia demonstrated inhibitor activity of alpha-amylase and alpha-glucosidase enzymes. In vitro studies reported that the ethanolic and ethyl acetate leaf and stem extracts of $M$. peltata (IC50 value 47.44-72.85 $\mu \mathrm{g} / \mathrm{mL}$ ) [90], the ethanolic leaf extract of $M$. hederacea $(91.44 \%$ inhibition) [18], and the hexane fraction of M. mammosa (66.19 $\pm 0.41 \%$ inhibition) [56] demonstrated good alpha-amylase inhibition. In all these studies, extracts from Merremia species had higher $\alpha$-glucosidase inhibition compared to the reference anti-diabetic drug (acarbose). In vivo studies using streptozotocin-induced diabetic male Wistar rats reported that the aqueous extract of $M$. tridentata roots [52], methanolic extract of M. emarginata whole plant [93], and ethanolic leaf extract of $M$. hederacea [18] demonstrated potent antidiabetic activities in streptozotocin-induced diabetic rats that were comparable to the reference drug (glibenclamide) used. Overall, the anti-diabetic effects exhibited by extracts of the Merremia species studied were attributed to their phytochemical constituents particularly flavonoids and phenolic compounds. Although the results of the in vitro and in vivo studies corroborate the ethnomedicinal claims of Merremia species as antidiabetic plants, it is recommended that longer duration studies on the chronic model are carried 
out to explicate the mechanism of action in order to develop these species as potential antidiabetic drugs.

\section{Antimicrobial Activities}

Several studies have shown that Merremia species have antibacterial and antifungal effects, and thus have the potential to be developed as antimicrobial agents. Different parts of Merremia species extracts show inhibitory actions on several bacteria and fungi to different degrees. The antibacterial activity of the methanolic leaf extracts of Convolvulaceae family members, including $M$. tridentata was assessed against two Gram-positive bacteria (Bacillus subtilis and Staphylococcus aureus) and two Gram-negative bacteria (Escherichia coli and Vibrio parahaemolyticus) [94]. The extracts had more antibacterial activity on Gram-positive bacteria (MIC range: $0.25-0.5 \mathrm{mg} \mathrm{mL}^{-1}$; zone formation range: $10 \mathrm{~mm}-14$ $\mathrm{mm}$ ) than Gram-negative bacteria (MIC range: $0.5-1 \mathrm{mg} \mathrm{mL}^{-1}$; zone formation range: $9 \mathrm{~mm}-11 \mathrm{~mm}$ ). Pavithra et al. [66] also reported that the methanol stem extracts of $M$. tridentata showed strong antibacterial activity only on Gram-positive bacteria (S. aureus and B. subtilis) with inhibition zones of $12.0 \mathrm{~mm}$ and $11.3 \mathrm{~mm}$, respectively. These extracts were evaluated for antibacterial activity using the broth dilution and disk diffusion assays. The extracts demonstrated antibacterial effects comparable to the standard drugs (ampicillin and gentamicin). The bactericidal effect was attributed to the extract's phytochemical constituents like alkaloids, triterpenoids, tannins, glycosides, and steroids. In another study, the aqueous, petroleum ether and methanol extracts (concentration range of $10 \mu \mathrm{g}$ $\mathrm{mL}^{-1}, 25 \mu \mathrm{g} \mathrm{mL}^{-1}$, and $50 \mu \mathrm{g} \mathrm{mL}^{-1}, 100 \mu \mathrm{g} \mathrm{mL}^{-1}$ ) of M. emarginata leaves showed antibacterial activities against B. cereus, E. coli, P. aeruginosa, and S. aureus [95]. The leaf extracts exhibited higher antibacterial activity (with a zone of inhibition range of 0.7-13.7 $\mathrm{mm}$ ) on all the bacteria compared with penicillin $(16.0-17.0 \mathrm{~mm})$. The observed antibacterial activity of $M$. emarginata leaf extracts was ascribed to the presence of chemical compounds such as flavonoids, glycosides, terpenoids, starch, and amino acids in the aqueous extract; flavonoids, tannins, carbohydrates, and amino acids in the methanol extract, and flavonoids, tannins, glycosides, carbohydrates, and amino acids in the petroleum ether extract. Diwan and Gadhikar [96] reported that all four extract solvents (aqueous, acetone, chloroform, and petroleum ether) of M. emarginata leaves were effective against bacteria (A. viscoscus, B. subtilis, E. coli, L. rhamnosus, S. aureus, S. epidermidis, and S. mutans) known to cause oral diseases in human. Acetone extract had maximum inhibitory $(24 \%)$ action against S. epidermidis and was the most effective. The extracts' antibacterial properties were imputed to their aromatic phytochemical contents (e.g., phenolics).

Using Luria-Bertani agar disk diffusion assay, Rameshkumar et al. [21] investigated the antibacterial activity of $M$. emarginata aqueous leaf extract, thioglycolic acid-capped cadmium telluride quantum dots (TGA-capped-CdTe QDs, hereafter), and their mixture (T-M complex, hereafter) $\left(1 \times 10^{-6} \mathrm{M}\right.$ and $100 \mu \mathrm{g}$, respectively) against a Gram-negative bacterium, E. coli. The T-M complex served as an antimicrobial agent. The results showed that the plant extract alone and T-M complex showed $10 \mathrm{~mm}$ and $16 \mathrm{~mm}$ zones of inhibition, respectively. Compared with $M$. emarginata extract, the antibacterial activity of the T-M complex was thought to be due to electrostatic interactions. The observed antimicrobial activity of the T-M complex was ascribed to the capability of TGA-capped-CdTe QDs and M. emarginata extract phytochemical constituents (e.g., alkaloids, flavonoids, phenolics, saponins, steroids, and terpenoids) to induce oxidative stress on the bacteria (E. coli) surface biomolecules thereby damaging its membrane. Merremia umbellata methanol leaf extract (1000 $\mu \mathrm{g} \mathrm{mL}^{-1}$ ) showed antibacterial activities with percentage inhibition of 32\%, 55\%, and $67 \%$ on Klebsiella pneumoniae (13883), S. aureus (25923), and Pseudomonas aeruginosa (27853), respectively [62]. The observed antibacterial effect was ascribed to phytochemicals such as leucoanthocyanidins, terpenes, and/or steroids and tannins present in the extract. The ethanol leaf extract of $M$. peltata showed a $5.7-\mathrm{mm}$ average zone of inhibition against B. subtilis and S. aureus (at $10 \mu \mathrm{g} \mathrm{mL}^{-1}$ and $20 \mu \mathrm{g} \mathrm{mL}^{-1}$, respectively), relative to the $2 \mathrm{~mm}$ by streptomycin. M. peltata ethanol leaf extract had $4.7-\mathrm{mm}$ and $2.7-\mathrm{mm}$ average zones of 
inhibition against P. aeruginosa and E. coli (at $15 \mu \mathrm{g} \mathrm{mL} L^{-1}$ and $5 \mu \mathrm{g} \mathrm{mL} \mathrm{L}^{-1}$, respectively), relative to the $1 \mathrm{~mm}$ by chloramphenicol [19].

Luciardi et al. [4] reported that $M$. dissecta methanol leaf extracts showed a high antibacterial effect against $P$. aeruginosa and biofilm inhibition effects against $P$. aeruginosa and S. aureus, compared with azithromycin which served as the control. The observed antibacterial activity was ascribed to lipophilic compounds present in the extract, suggesting that the extract was toxic to cell membranes by causing loss of chemiosmotic control. It was suggested that volatile plant metabolites, like spathulenol, present in the extracts may be responsible for decreasing the biosynthesis of $\mathrm{N}$-acyl homoserine lactone by $72 \%$, attenuating the expression of virulence factors such as elastase activity $(27 \%)$ and biofilm production (55\%) in P. aeruginosa.

Studies on antifungal activities have only been done on M. tridentata and M. borneensis. In both studies, although the antifungal activities were tested on mould and yeast fungal strains, both species only showed antifungal activity on yeast. The methanol leaf extract of M. tridentata (at $0.01 \mathrm{mg} \mathrm{mL}^{-1}, 0.05 \mathrm{mg} \mathrm{mL}^{-1}, 0.1 \mathrm{mg} \mathrm{mL}^{-1}, 0.25,0.5 \mathrm{mg} \mathrm{mL}^{-1}$ and $1 \mathrm{mg}$ $\mathrm{mL}^{-1}$ ) showed antifungal activity on yeast (MIC range: $0.25-0.5 \mathrm{mg} \mathrm{mL}^{-1}$; zone formation range: $10-15 \mathrm{~mm}$ ) [94]. Zulhamizan et al. [63] reported that methanol leaf extract of $M$. borneensis showed antifungal activity against $S$. cerevisiae with increasing concentration (60-400 $\left.\mathrm{mg} \mathrm{mL}^{-1}\right)$ and the inhibition zones ranged between $5.5-15.5 \mathrm{~mm}$. The observed antifungal activity was attributed to the presence of palmitic acid in the methanol leaf extract only.

The above studies suggest that Merremia species possess antimicrobial (antibacterial and antifungal) properties. However, the studies are in vitro studies and may not show the same effects in in vivo investigations. For more reliable and valid results, the antimicrobial activities of Merremia species extracts should be investigated in in vivo models infected with clinically isolated strains and specific microorganisms to demonstrate efficacy and elucidate their mechanism of action.

\section{Anti-Influenza Activity}

Influenza is a highly transmissible and widespread respiratory disease that is caused by influenza viruses and occurs throughout the year in tropical areas [97]. According to WHO, global influenza epidemics result in 3-5 million severe illness cases and 290,000-650,000 mortalities yearly [98]. Several anti-influenza viral drugs including Zanamivir are used for treating influenza viral infection. However, the emergence of resistance of viruses to these drugs, as well as adverse side effects, has been reported [97]. Natural products with good tolerability and greater efficacy than existing anti-influenza drugs are of interest in the treatment of influenza virus infection. Only one report is available on the anti-influenza activity of Merremia species. Using hemagglutinin assay, the anti-influenza A (subtype H1N1) activity of ethyl acetate fraction of methanol extract of M. mammosa tuber was evaluated on embryonic chicken eggs injected with the H1N1 virus [99]. The virus subtype H1N1 (with no treatment extract) and Zanamivir $\left(10 \mu \mathrm{g} \mathrm{mL} \mathrm{m}^{-1}\right)$ served as negative and positive controls, respectively. The result showed that the ethyl acetate fraction of the $M$. mammosa tuber extract $\left(1000 \mu \mathrm{g} \mathrm{mL}{ }^{-1}\right)$ had an anti-influenza effect similar to Zanamivir, reducing hemagglutinin virus titer by $94 \%$ and $100 \%$, respectively. The activity was attributed to the flavonoid and terpenoids contents of the ethyl acetate fraction. The result indicates that M. mammosa tuber extract may be a potential alternative medicine for the treatment of the influenza virus. However, this is an in vitro study, in vivo studies are required to evaluate whether $M$. mammosa tuber extract will result in antiviral activity in vivo and to elucidate the mechanism of action. Furthermore, other species in the genus should be investigated for their anti-influenza activities.

\section{Anti-Inflammatory Activity}

Inflammation is a defense mechanism of the body's immune system against unwanted foreign substances, tissue injury, or pathogens that attack tissue cells. However, unreg- 
ulated inflammation often results in chronic inflammation, consequently leading to the development of various chronic cardiovascular and pulmonary diseases [100]. Several pro-inflammatory mediators such as inducible cyclooxygenase enzyme (COX-2) and tumor necrosis factor-alpha (TNF- $\alpha)$ are usually observed in numerous inflammatory diseases, including inflammatory vascular diseases and rheumatoid arthritis [101]. Medicinal plants that can selectively and effectively inhibit the expression or activation of these pro-inflammatory mediators are of significant clinical importance. The anti-inflammatory activity of orally administered whole plant $M$. tridentata extracts (water, ethanol, benzene, petroleum ether, and chloroform) on male albino rats using the carrageenan-induced rat paw inflammation method was assessed [55]. Normal saline and indomethacin were used as the control and standard drug, respectively. The extracts inhibited paw volume at a range of 15.6-38.3\%; the ethanol extract was the most effective with $38.3 \%$ and $42.8 \%$ inflammation inhibition at $100 \mathrm{mg} \mathrm{kg}^{-1}$ and $200 \mathrm{mg} \mathrm{kg}^{-1}$ doses, respectively, relative to that the standard drug (48.5\%) after $3 \mathrm{~h}$. The ethyl acetate extract of $M$. emarginata at a concentration of $50 \mu \mathrm{g} \mathrm{mL} \mathrm{m}^{-1}$ effectively inhibited TNF- $\alpha$ production $\left(\mathrm{IC}_{50}\right.$ of $\left.5.9 \mu \mathrm{g} \mathrm{mL}^{-1}\right)$ in lipopolysaccharide (LPS)-induced human acute monocytic leukemia cells (THP-1) [3]. In another study, the anti-inflammatory activity of different extracts $\left(250 \mathrm{mg} \mathrm{kg}^{-1}\right.$ of petroleum ether and $300 \mathrm{mg} \mathrm{kg}^{-1}$ of ethyl acetate, solvent ether, butanol, and butanone) fractionated from M. tridentata ethanol roots extract was tested by the authors of [53] in albino mice using the carrageenan-induced rat paw edema method. Orally administered Tween 80 solution and aspirin served as the control and standard drug, respectively. The results showed that solvent ether, ethyl acetate, butanol, and butanone fractions were more effective $(87 \%$, $84 \%, 72 \%$, and $68 \%$ at $300 \mathrm{mg} \mathrm{kg}^{-1}$, respectively) at inhibiting rat paw edema, relative to the standard (59.7\%) and control (no inhibition). The anti-inflammatory activity was attributed to the antimicrobial property of flavonoids present in the extracts. Using the complete Freund's adjuvant-induced arthritis model, Kamalutheen et al. [55] reported that the methanolic extract of $M$. tridentata whole plant demonstrated $49.0 \%$ and $51.7 \%$ anti-arthritic activity at $100 \mathrm{mg} \mathrm{kg}^{-1}$ and $200 \mathrm{mg} \mathrm{kg}^{-1}$ on male albino rats. The anti-arthritic activity of the extract was comparable to the standard drug (indomethacin) (55.5\%). In another in vitro anti-arthritic study using the protein denaturation inhibition method, at $250 \mu \mathrm{g} \mathrm{mL}^{-1}$, the ethanol extract of M. emarginata whole plant and its methanol and ethyl acetate fractions exhibited high protein denaturation inhibition percentages of $95.4 \%, 87.7 \%$, and $72.6 \%$, respectively, which was comparable with the standard (diclofenac sodium) (98.4\%) [102]. However, using this same protein denaturation inhibition technique in a recent study, the aqueous portion of fractionated methanol extract (APFME) of $M$. vitifolia leaves only showed anti-arthritic activity of $64 \%$ at a higher concentration of $500 \mu \mathrm{g} \mathrm{mL}^{-1}$ [20].

Collectively, these findings demonstrate that the investigated Merremia species extracts possess remarkable in vitro and in vivo anti-inflammatory activities, which support the traditional use of some of these species in the treatment of various inflammatory diseases.

\section{Anti-Nociceptive Activity}

Pain is an unpleasant sensory and emotional experience linked with or resembling that related to actual or potential tissue damage [103]. To relieve pain, non-steroidal antiinflammatory drugs such as salicylates and acetic acid derivatives, and opioids are used. However, the prolonged use of these medications leads to decreased gastrointestinal system function [104]. Antinociceptive drugs developed from plant extracts with little or no side effects are of interest in modern medicine. Using formalin-induced paw licking (at early and late stages) and acetic acid-induced writhing tests, the in vivo antinociceptive activity of M. vitifolia APFME (200 mg kg-1 and $400 \mathrm{mg} \mathrm{kg}^{-1}$ BW, PO) was assessed in Swiss Albino mice [20]. Diclofenac sodium (10 $\mathrm{mL} \mathrm{kg}^{-1} \mathrm{BW}$, administered intraperitoneally (IP)) served as the standard drug. In the formalin-induced paw licking test, the findings of the study demonstrated that the APFME at $200 \mathrm{mg} \mathrm{kg}^{-1}$ and $400 \mathrm{mg} \mathrm{kg}^{-1}$ BW PO showed dose-dependent higher anti-nociceptive activity ( $44 \%$ and $26 \%, 30 \%$, and $20 \%$ in both early and late test stages, respectively) relative to the control ( $20 \%$ and $15 \%$, respectively) 
at the same concentrations. Similarly, reduced (44\% and 30\%, respectively) abdominal contortions with increasing doses of APFME relative to the control (21\%) were observed in the acetic acid-induced writhing test. Future studies should investigate more species in the genus for their antinociceptive activity and attempt to identify pure compounds and elucidate the mechanism of action responsible for the activity.

\section{Thrombolytic Activity}

Several thrombolytic drugs including streptokinase, are used to treat acute coronary disorders resulting from thrombosis [20]. However, there are reports of hypertension and severe hemorrhagic transformation resulting from the use of thrombolytic drugs [20]. Alternative medicines from medicinal plants with thrombolytic activity are increasingly being researched for their safety profile to overcome the side effects resulting from modern thrombolytic drugs. Only one report, however, is available on the thrombolytic activity of Merremia species. The aqueous portion of fractionated methanol extract of $M$. vitifolia leaves $\left(500 \mu \mathrm{g} \mathrm{mL}^{-1}\right)$ was reported to demonstrate significant thrombolytic activity $(42.5 \%$ clot lysis) in blood samples from male and female adult human volunteers as compared with the negative control (normal saline; 4.80\%) [20]. However, the positive control (streptokinase) demonstrated much higher thrombolytic activity $(72.2 \%)$ than $M$. vitifolia. This study was assessed in blood samples from male and female (1:1) adult human volunteers with no anticoagulant and oral contraceptive treatments history. The inhibition of clot formation in the subjects was attributed to various phytochemicals (alkaloids, flavonoids, tannins, and triterpenoids) in M. vitifolia. Studies to identify the major compound responsible for this activity, as well as its mechanism of action, should be conducted. Although M. vitifolia leaf extract gave low thrombolytic activity as compared with the positive control in this study, other species in the genus may give higher thrombolytic activity if evaluated. Future studies should evaluate these species for thrombolytic activity.

\section{Anti-Urolithiatic Activity}

Urolithiasis (commonly referred to as kidney stones) is the formation of solid particles anywhere in the urinary tract and it is the most prevalent type of all urinary stone diseases [105,106]. Usually, the solid particles are very small and can dissolve and leave the body without any problem. However, the blockage of the flow of urine by even a small stone results in excruciating pain, which requires immediate medical attention [106]. Several pharmaceutical drugs and medical technologies such as percutaneous nephrolithotomy and extracorporeal shock wave lithotripsy are available to treat and prevent urolithiasis. However, these medications and techniques are expensive and there are risks of side effects and reoccurrence of urolithiasis even with medication [106]. Several medicinal plants, including Merremia species, have the potency to inhibit stone formation as well as break formed stones.

A dissolution model study was carried out by Neeraja Kamakshi et al. [107] on the anti-urolithiatic activity of methanol extracts of the M. emarginata plant. Artificial stones (calcium phosphate and calcium oxalate) were produced by homogenous precipitation, while eggs' semi-permeable membrane served as dissolution bags; Cystone ${ }^{\circledR}$ served as a positive control. M. emarginata showed anti-urolithiatic activity in a dose-dependent manner (with calcium phosphate and calcium oxalate mineralization inhibition of $81 \%$ and $84 \%$, respectively, relative to $91 \%$ by the standard drug at $400 \mu \mathrm{g} \mathrm{mL}^{-1}$ ). The observed bioactivity was attributed to the presence of phytochemicals such as alkaloids and flavonoids in the plant extract. The result implies that the methanolic extract of the $M$. emarginata plant has great potential to be developed as a pharmaceutical agent for the treatment of urolithiasis. However, in vivo and clinical studies are required to clarify the mechanism of action and identify the exact compound responsible for this activity. Other species in the genus should be investigated for their anti- urolithiatic activities. 


\section{Nephroprotective Activity}

Nephrotoxicity usually results from the frequent use of therapeutic and chemotherapeutic drugs like aminoglycoside antibiotics (gentamicin) and cisplatin, chronic diseases, and exposure to heavy metals. These causes often result in End-Stage Renal Disease (ESRD) and death. The few therapeutic drugs currently available to treat nephrotoxicity only delay and do not stop the progression of nephrotoxicity [108]. There is increasing research focus on medicinal plants with nephroprotective activities that can be used as safer and better alternatives in treating nephrotoxicity. The nephroprotective activity of ethanol extract of M. emarginata leaves $\left(150,200\right.$, and $\left.250 \mathrm{mg} \mathrm{kg}^{-1} \mathrm{BW}, \mathrm{PO}\right)$ in adult albino Wistar rats was investigated by Rameshkumar et al. [64] using a histopathology examination. Nephrotoxicity was induced with gentamicin $\left(20 \mathrm{mg} \mathrm{kg}^{-1} \mathrm{BW}\right.$, IP); normal drinking water $\left(10 \mathrm{mg} \mathrm{kg}^{-1} \mathrm{BW}\right)$ served as control. The results revealed that the extracts caused regeneration of glomerular, tubular, and proximal tubular epithelial cells of damaged kidneys in M. emarginata extract-treated rats (especially at $250 \mathrm{mg} \mathrm{kg}^{-1}$ ). The leaf extract's nephroprotective activity was ascribed to its antioxidant (polyphenolics) contents. The result supports the traditional use of M. emarginata leaves in treating kidney disorders. Further studies should be carried out to determine the exact polyphenolics and their mechanism against nephrotoxicity. Other Merremia species (M. tridentata and M. vitifolia) that are used in traditional medicines to treat various kidney and urinary disorders should be investigated for their nephroprotective activities.

\section{Diuretic/Blood Pressure-Lowering Activity}

Diuretics are medications that elevate the amount of water and salt expelled from the body as urine and are widely used in the treatment of kidney and liver diseases, edema, hypertension, and congenital heart failure [79]. Commercial diuretic drugs have been reported to be associated with many side effects (such as dehydration, hypokalemia, fever, bleeding, and cough). Diuretic drugs of medicinal plant origins without harmful effects can be considered a better alternative to commercial drugs. The angiotensinconverting-enzyme inhibitory (ACEI), diuretic, and hypotensive effects of the aqueous methanol crude extract of $M$. emarginata aerial parts administered intravenously were tested in Sprague-Dawley rats by the authors of [109]. Dimethyl sulphoxide and captopril served as the control and standard drugs, respectively, for the ACEI test. For the diuretic test, normal saline and frusemide $\left(10 \mathrm{~mL} \mathrm{Kg}^{-1}\right.$, IP) served as control and standard drug, respectively. M. emarginata extract showed serum ACEI activity $\left(\mathrm{IC}_{50}\right.$ value of $422 \mu \mathrm{g} \mathrm{mL}^{-1} ; 2.0 \mathrm{mg} \mathrm{mL}^{-1}$ extract had the highest (81\%) ACEI effect compared with captopril $(89 \%)$ ). Additionally, the extract increased the volume of urine and excretion of urinary $\mathrm{Na}^{+}$at $30 \mathrm{mg} \mathrm{Kg}^{-1}$ and $50 \mathrm{mg} \mathrm{Kg}^{-1}$ doses. The extract's ACEI activity was ascribed to the presence of phytochemicals (like tannins, flavonoids, and alkaloids) via enzyme metal co-factor sequestration, protein precipitation, or other mechanisms. Similarly, the diuretic activity was ascribed to the phytochemical (such as alkaloids and phenolics) contents of M. emarginata extract. The extract exhibited a dose-dependent drop in the average arterial blood pressure range of $21.5-61.7 \%$ at a concentration range of $0.1-3.0 \mathrm{mg} \mathrm{Kg}^{-1}$. The authors alluded to vasodilation with heightened cardiac output to have led to a remarkable decrease in diastolic blood pressure. Additionally, it was reported that the plant extract caused heart rate decline, which was attributed to reflex mechanism involvement. Other mechanisms, such as indirect and/or direct effect on heart, inhibition, and/or relaxation of vascular smooth muscle contraction, leading to decreased total peripheral resistance or combination of mechanisms, were suggested.

In an in vivo study, the diuretic activity of the aqueous extract of $M$. emarginata leaves (200, 400, and $600 \mathrm{mg} / \mathrm{kg}$ b.w.) was investigated in adult female Wistar albino rats [79]. Normal drinking water $(10 \mathrm{~mL} / \mathrm{kg}$ b.w) and a commercial diuretic drug (furosemide $20 \mathrm{mg} / \mathrm{kg}$ b.w) served as controls. The diuretic activity of the extract was confirmed by analyzing the disparity in the total volume of urine and diuretic markers, compared to the controls. Merremia emarginata leaf extract demonstrated a significantly higher diuretic 
effect in treated rats compared to the control group rats. This diuretic activity was without side effects such as proteinuria or glycosuria. Furthermore, a polyphenolic compound (chlorogenic acid) (6) was identified through ultra-performance liquid chromatographytandem mass spectrometry (UPLC-MS/MS) and reverse phase-high performance liquid chromatography (RP-HPLC) to be responsible for the diuretic activity. These results suggest that $M$. emarginata may potentially act as a good diuretic agent without causing harmful side effects.

\section{Wound Healing Property}

Wounds result from physical injuries, that lead to a break or opening of the skin [110]. Proper wound healing is important to restore the disrupted functional status of the skin, as well as the interrupted anatomical continuity [110]. Several medicinal plants are used in traditional medicine to treat wounds because of their wound-healing properties $[110,111]$. Such medicinal plants promote wound healing by the activities of their bioactive substances (e.g., flavonoids, phenolic acids, tannins, etc.). The bioactive compounds in medicinal plants exhibit exceptional fast healing by reducing lipid peroxidation, thereby improving vascularity and preventing or slowing the onset of necrosis, and initiating skin cell differentiation $[110,111]$. The wound-healing activity of different ethanol extract fractions (solvent ether, petroleum ether, ethyl acetate, butanol, and butanone) of M. tridentata roots was tested in albino mice by Bidkar et al. [53] using the tensile strength of wound models (re-sutured incision and grass pith granuloma) on the 10th day after wounding. Orally administered Tween 80 solution served as the control. The results showed that all extract fractions $\left(250 \mathrm{mg} \mathrm{kg}^{-1}\right.$ or $\left.300 \mathrm{mg} \mathrm{kg}^{-1}\right)$ exhibited considerably higher tensile strength $(215.4 \mathrm{~g}, 213 \mathrm{~g}, 243.7 \mathrm{~g}, 236.1 \mathrm{~g}$, and $232.9 \mathrm{~g}$, respectively) than the control $(144.3 \mathrm{~g})$ in the re-sutured incision model. Similarly, all extract fractions exhibited considerably higher tensile strength ( $221.7 \mathrm{~g}, 210 \mathrm{~g}, 264.1 \mathrm{~g}, 332.8 \mathrm{~g}$, and $335.7 \mathrm{~g}$, respectively) than the control $(155.4 \mathrm{~g})$ in the granuloma model. The wound-healing effect was attributed to the astringent property of flavonoid (present in the extracts), which allowed for wound contraction and enhanced epithelialization rate.

Sakinah et al. [112] fractionated M. mammosa ethanol extract and used $25 \mathrm{mg}$ of ethyl acetate, $n$-hexane, and water fractions to evaluate the wound-healing properties of M. mammosa plant in diabetic male Wistar rats. Diabetes was induced in rats by administering streptozotocin ( $\left.40 \mathrm{mg} \mathrm{kg}^{-1} \mathrm{BW}, \mathrm{IP}\right)$, while the wound was excised using the Morton method; aqua dest and gentamicin served as negative and positive controls, respectively. The ethyl acetate, $\mathrm{n}$-hexane and water fractions of M. mammosa extract showed the smallest wound diameter $(72 \%, 62 \%$, and $54 \%$, respectively) compared with the negative control $(114 \mathrm{~mm})$ on day 11 . Additionally, M. mammosa extract fractions showed wound reduction ( $89 \%, 90 \%$, and $93 \%$ in ethyl acetate, n-hexane, and water fractions, respectively) similar to the positive control (92\%) and higher than the negative control (82\%) on day 11 . It was suggested that the anti-inflammatory effect of the flavonoid glycoside content of M. mammosa extract fractions might be responsible for the wound-healing property.

In another study, Marchianti et al. [57] investigated the wound-healing potency of $1.5 \%$ M. mammosa plant gel formulations (incorporation of hydroxypropylmethylcellulose (HPMC), Carbopol, or sodium carboxymethylcellulose (Na CMC) into 10\% water fraction of the plant's ethanol extract) in diabetic Wistar rats by percentage wound size reduction, vascular endothelial growth factor expression, hydroxyproline levels, and histopathology assessments. Diabetes was induced by streptozotocin $\left(40 \mathrm{mg} \mathrm{kg}^{-1} \mathrm{BW}, \mathrm{IP}\right)$, while the wound was excised using the Morton method. The rats were divided into five treatment groups, viz. distilled water (negative control), neomycin sulfate and placenta extract gel (positive control), and $10 \%$ water fraction of M. mammosa extract in the gelling agents (3. HPMC, 4. Carbopol, and 5. Na CMC). All three M. mammosa gel formulations showed improved healing than the negative control and similar healing effect relative to the positive control in terms of optimum level vascular endothelial growth factor expression, hydroxyproline levels, and collagen density. The improved wound-healing effect of the 
three gel formulations was ascribed to the activities of flavonoid, as well as resin glycosides present in M. mammosa water fraction, restoring the delayed diabetic wound-healing process. The different investigations on the wound healing properties of Merremia species show that the extracts and fractions have potent wound healing abilities which support the traditional use of these species in treating wounds and inflammation.

\section{Antioxidant Activity}

Usually, the body keeps up a balance between the production of free radicals and their elimination. However, overproduction of reactive oxygen species leads to a disproportion between pro and antioxidants, resulting in oxidative stress [113]. Oxidative stress disrupts and damages DNA, cell structures, proteins, and lipids in the body and consequently results in neurodegenerative diseases, cancer, and diabetes, among others $[113,114]$. Antioxidants are useful chemical compounds that can reduce free radicals, decrease the rate of production, and even quench free radicals in the body [115]. Plants, including Merremia species, are rich sources of antioxidants. The antioxidant activities of the crude extracts (ethyl acetate, hexane, methanol, and $25 \%$ aqueous methanol) of $M$. emarginata whole plant were evaluated by 2,2-diphenyl-1-picrylhydrazyl (DPPH) and superoxide radical scavenging activity methods [116]. Vitamin C served as the antioxidant standard. Of the different extracts tested, the greatest potential scavenging activity was found to be the methanol crude extract $\left(\mathrm{IC}_{50} 8.6 \mu \mathrm{g} \mathrm{mL}{ }^{-1}\right)$. However, the scavenging activity of the reference drug $\left(\mathrm{IC}_{50} 3.3 \mu \mathrm{g} \mathrm{mL}{ }^{-1}\right)$ was higher than all extracts tested. The authors inferred that the phytochemical constituents of $M$. emarginata were responsible for the observed bioactivity. In another study, the antioxidant activity of $50-200 \mu \mathrm{g} \mathrm{mL}^{-1}$ solvent extracts (acetone, chloroform, methanol, and hot water) of $M$. tridentata roots and aerial parts were evaluated using DPPH, 2,2' -azino-bis (3-ethylbenzothiazoline-6-sulfonic acid) (ABTS), ferric reducing antioxidant power (FRAP), phosphomolybdenum reduction, ferrous ion $\left(\mathrm{Fe}^{2+}\right)$ chelation, $\beta$-carotene/linoleic acid peroxidation inhibition, and antihemolytic activity assays [54]. Acetone root extract exhibited a higher antioxidant activity (IC 5026.6 $\mu \mathrm{g} \mathrm{mL}^{-1}$ ) than that of $\alpha$-tocopherol standard in the DPPH assay. Compared to other extracts, $M$. tridentata acetone root extract exhibited the highest total antioxidant activity $\left(26,270.8 \mu \mathrm{mol} \mathrm{g}^{-1}\right)$ in the ABTS assay, highest ferric reducing antioxidant activity (2656.7 mmol Fe (II) $\mathrm{mg}^{-1}$ ) in the FRAP assay, and the strongest phosphomolybdenum reduction (56.7 $\mathrm{g}$ ascorbic acid/100 g) in the phosphomolybdenum reduction assay. The reducing power activity of the extracts was linked to high levels of phenolics. Also, the results showed a concentration-dependent $\mathrm{OH}^{\bullet}$ scavenging activity (range: $29.6-59.3 \%$ and $34.8-$ $52.9 \%$ by the root and aerial parts extracts at $200 \mu \mathrm{g} \mathrm{mL}{ }^{-1}$ ). In the $\mathrm{Fe}^{2+}$ chelation assay, the hot water extract of $M$. tridentata aerial parts exhibited the highest activity $(8.0 \mathrm{mg}$ EDTA g $^{-1}$ extracts). All the plant extracts inhibited $\beta$-carotene bleaching (range: $20.7-36.1 \%$ by the root extract and $13.3-32.9 \%$ by the aerial parts extract at $200 \mu \mathrm{g} \mathrm{mL}^{-1}$ ); acetone extracts (aerial parts and roots) and methanol root extract exhibited activities $(32.9 \%, 36.1 \%$, and $31.8 \%$, respectively) comparable with BHA standard (36.6\%). The distinct activity of M. tridentata acetone extract was suggested to be due to its high polyphenolics content. All the extracts showed higher peroxidation inhibition activity (aerial parts: $13.3-32.9 \%$, roots: $20.7-36.1 \%$ at $200 \mu \mathrm{g} \mathrm{mL}^{-1}$ ) compared with $\alpha$-tocopherol standard. The results of the antihemolytic activity assay showed that the acetone extract of $M$. tridentata roots was the most effective with $82.7 \%$ red blood cell hemolysis inhibition. The methanol and acetone extracts of the aerial parts showed comparable (70.5\% and $74.0 \%$, respectively) hemolysis inhibiting activity. The results of this study clearly show that the various solvent extracts of $M$. tridentata roots and aerial parts (particularly the acetone root extract) possess significant free radical scavenging and antioxidant activities. In another similar study, the antioxidant potency of different concentrations of aqueous extract of $M$. emarginata leaves was carried out in an in vitro study using reducing power, DPPH, ABTS, superoxide anion scavenging, and lipid peroxidation inhibition assays [21]. The authors also evaluated the leaf extract's antioxidant activity based on fluorescence quenching using TGA-capped-CdTe QDs as 
fluorescent probes. The results showed that the reducing power of M. emarginata leaf extract increased proportionally to concentration. The extract showed optical density (0.79) comparable to butylated hydroxytoluene standard (1.32) at $1000 \mu \mathrm{g} \mathrm{mL}^{-1}$. The leaf extract's reducing power was attributed to reductones' presence, stabilizing and terminating radical chain reactions. The DPPH assay results showed that the leaf extract had antioxidant potency $\left(\mathrm{IC}_{50}\right.$ value of $86.5 \mu \mathrm{g} \mathrm{mL}{ }^{-1}$ ) relative to butylated hydroxytoluene standards ( $\mathrm{IC}_{50}$ value of $23.4 \mu \mathrm{g} \mathrm{mL}^{-1}$ ), and the effect was ascribed to its antioxidant (e.g., flavonoids and polyphenolics) contents. In the ABTS assay, the extract $\left(100 \mu \mathrm{g} \mathrm{mL}^{-1}\right)$ showed ca. $71 \%$ inhibition (with $\mathrm{IC}_{50}$ value of $30.1 \mu \mathrm{g} \mathrm{mL}^{-1}$ ) relative to butylated hydroxytoluene standards $\left(\mathrm{IC}_{50}\right.$ value of $27.2 \mu \mathrm{g} \mathrm{mL}^{-1}$ ). The leaf extract showed superoxide anion scavenging activity $\left(\mathrm{IC}_{50}\right.$ value of $40.3 \mu \mathrm{g} \mathrm{mL}^{-1}$ ). The results of the TGA-capped-CdTe QD assay showed fluorescence emission quenching by M. emarginata extract. The quenching of fluorescence emission was attributed to the prevention of the electron-hole recombination process, causing a decrease in the fluorescence intensity due to the trapping of TGA-capped-CdTe QD holes by M. emarginata leaf extract.

Purushoth et al. [102] also subjected M. emarginata whole plant ethanol extract and its fractions (chloroform, ethyl acetate, hexane and methanol) to an in vitro antioxidant potency test using $\alpha, \alpha$-diphenyl- $\beta$-picrylhydrazyl (DPPH) scavenging, hydrogen peroxide scavenging, ABTS scavenging, and hydroxyl radical in the para-nitroso dimethylaniline assays. The DPPH assay results showed that the extracts had moderate to high antioxidant potency. However, the ethanol extract and methanol fraction had the highest antioxidant potency ( $\mathrm{IC}_{50}$ values of $26.5 \mu \mathrm{g} \mathrm{mL}^{-1}$ and $27.5 \mu \mathrm{g} \mathrm{mL}^{-1}$, respectively). All extracts showed high antioxidant activity in the ABTS assay with $\mathrm{IC}_{50}$ values range of $15-38 \mu \mathrm{g} \mathrm{mL}-1$ relative to ascorbic acid and rutin standards $\left(\mathrm{IC}_{50}\right.$ values of $18.5 \mu \mathrm{g} \mathrm{mL}-1$ and $12.7 \mu \mathrm{g}$ $\mathrm{mL}^{-1}$, respectively). The antioxidant potency of the essential oil and different extracts (aqueous ethanol, butanol, chloroform, ethyl acetate, and hexane) of $M$. borneensis leaves and stems measured by reducing power (phosphomolybdenum method), $\beta$-carotenelinoleate model system, and DPPH radical scavenging assays revealed that all M. borneensis extracts showed antioxidant potency. However, the aqueous ethanol extracts showed the highest antioxidant potency in all the assays (with $31 \%$ antioxidant capacity in the phosphomolybdenum method, $84 \%$ inhibition of $\beta$-carotene bleaching, and $80 \% \mathrm{DPPH}$ radical scavenging activity at $100 \mu \mathrm{g} \mathrm{mL}^{-1}$ ). It was suggested that antioxidant responses might be due to the quantity and/or variety of phenolics present in the leaf extracts of M. borneensis [22]. In another study by Joshi et al. [117], the antioxidant potential of methanol extract of different parts (callus, leaf, seed and stem) of $M$. dissecta and M. aegyptia was evaluated using DPPH free radical scavenging assay; ascorbic acid was used as a standard. The study revealed an increasing free radical scavenging activity with increasing plant extracts concentration in both Merremia species. The leaf, seed, and stem extracts of M. dissecta had $75 \%, 90 \%$, and $93 \%$ free radical scavenging effects with $\mathrm{IC}_{50}$ values of 82 , 80 and $61 \mu \mathrm{g} \mathrm{mL}{ }^{-1}$, respectively, while the seed extract of $M$. aegyptia had a free radical scavenging effect of $90 \%$ comparable to ascorbic acid with $92 \%$ at $500 \mu \mathrm{g} \mathrm{mL}^{-1}$. The authors attributed the observed antioxidant activity to the possible presence of other bioactive compounds like ascorbic acid, tocopherol, and pigments in the plants since the flavonoid contents did not correlate with observed antioxidant activity. To assess the pharmacological properties of a Thai traditional herbal formula (Sahatsatara), Thamsermsang et al. [118] used 2,2-dipheny-1-picrylhydrazyl (DPPH) assay to test the free radical scavenging activity of the ethanol extracts $\left(3.75 \mu \mathrm{g} \mathrm{mL}^{-1}, 15 \mu \mathrm{g} \mathrm{mL}^{-1}, 30 \mu \mathrm{g} \mathrm{mL}^{-1}, 60 \mu \mathrm{g} \mathrm{mL}^{-1}, 120 \mu \mathrm{g} \mathrm{mL}^{-1}\right)$ of its 21 ingredients, including M. vitifolia stem. Gallic acid, L-ascorbic acid and piperine were used as references. M. vitifolia showed a dose-dependent free radical scavenging ability, ranking fourth (with the $\mathrm{IC}_{30}$ value of $25 \mu \mathrm{g} \mathrm{mL}{ }^{-1}$ ) relative to other components. The free radical scavenging activity of Sahatsatara was thus attributed to gallic acid and other unidentified bioactive compounds present in the formula's components.

In a recent study, the antioxidant potency of $2 \mathrm{mg} \mathrm{GAE} \mathrm{mL}^{-1}$ of hexane, ethyl acetate and methanol extracts of M. mammosa plant was tested using DPPH, hydroxyl radical and 
superoxide anion scavenging methods [56]. In the DPPH assay, M. mammosa methanol extract showed higher antioxidant activity relative to the positive control with $\mathrm{IC}_{50}$ values of $0.7 \mathrm{GAE} \mathrm{ml}^{-1}$ and $0.9 \mathrm{GAE} \mathrm{ml}^{-1}$, respectively. In the hydroxyl radical scavenging assays, $M$. mammosa methanol extract exhibited higher antioxidant activity relative to positive control with $92 \%$ and $89 \%$ inhibition values, respectively. The superoxide anion assay showed that $M$. mammosa methanol extract had higher antioxidant activity relative to hexane and ethyl acetate extracts (with inhibition values of $25 \%, 20 \%$, and $19 \%$, respectively). In a more recent study, the antioxidant activity of different extracts (N-Heksan, ethyl acetate, and methanol) of M. peltata leaf and stem was determined by DPPH radical scavenging and FRAP methods [90]. Ascorbic acid served as the standard antioxidant. Of all the sample extracts, the methanolic stem extract showed the best antioxidant activity $\left(\mathrm{IC}_{50}\right.$ values $47.37 \mu \mathrm{g} / \mathrm{mL}$ ) in the DPPH radical scavenging assay as well as the highest total antioxidant power (with value $207.08 \mu \mathrm{mol} / \mathrm{g}$ ) in the FRAP assay. However, these activities were still lower than that of ascorbic acid with an $\mathrm{IC}_{50}$ value of $10.49 \mu \mathrm{g} / \mathrm{mL}$ in the DPPH assay and a total antioxidant power value of $340.04 \mu \mathrm{mol} / \mathrm{g}$ in the FRAP assay.

Collectively, the results from the different antioxidant potency evaluations of Merremia species indicate that the extracts are effective as natural antioxidants. None of the extracts tested demonstrated weak or inactive antiradical potential, rather the antioxidant activities displayed could be classified as either very strong, strong, and moderate. Antioxidant activity is classified into four groups based on $\mathrm{IC}_{50}$ values; very strong $(<50 \mu \mathrm{g} / \mathrm{mL})$, strong $(50-100 \mu \mathrm{g} / \mathrm{mL})$, moderate $(101-250 \mu \mathrm{g} / \mathrm{mL})$, weak $(251-500 \mu \mathrm{g} / \mathrm{mL})$ and inactive ( $>500 \mu \mathrm{g} / \mathrm{mL}$ ) [119]. Hence, Merremia species extracts can be utilized for use as dietary supplements or as functional ingredients in nutraceutical and pharmaceutical products.

\section{Insecticidal Activity}

The development of plant-based insect repellents that are effective, non-toxic, and have a short half-life will benefit both farmers and the environment by preventing the spread of vector-borne diseases $[120,121]$. The exploitation of bioactive constituents in plants with insecticidal activity are being considered as alternatives to chemical insecticides and pesticides as a result of their lower persistence in the environment, as well as low toxicity effects on non-target organisms [120,121]. Some Merremia species have been investigated for their insecticidal activities. Oliveira et al. [122] screened 94 extracts from 10 plants, including the extracts $\left(250 \mu \mathrm{g} \mathrm{mL} \mathrm{m}^{-1}\right.$ of acetone and its fractions (hexane, chloroform, ethyl acetate, and methanol)) of M. aegyptia leaves, for larvicidal activity against Aedes aegypti commonly found in the Northeast region of Brazil. Dimethyl sulfoxide (DMSO) and Temephos (a synthetic insecticide) served as the negative control and positive controls, respectively. It was reported that nine extracts, including acetone extract and the hexane fraction of M. aegyptia leaves exhibited over $100 \%$ activity (with $\mathrm{LD}_{50}$ values of $120.7 \mu \mathrm{g} \mathrm{mL}^{-1}$ and $144.3 \mu \mathrm{g} \mathrm{mL}^{-1}$, respectively) against the mosquitoes fourth instar larvae. The larvicidal activity was suggested to be due to the presence of bioactive compounds.

In another study, the larvicidal activity of aqueous extract $\left(70 \mu \mathrm{g} \mathrm{mL}^{-1}, 140 \mu \mathrm{g} \mathrm{mL} \mathrm{m}^{-1}\right.$, $210 \mu \mathrm{g} \mathrm{mL}-1,280 \mu \mathrm{g} \mathrm{mL}$, and $350 \mu \mathrm{g} \mathrm{mL} \mathrm{mL}^{-1}$ ) and silver nanoparticles $\left(4 \mu \mathrm{g} \mathrm{mL}^{-1}, 8 \mu \mathrm{g} \mathrm{mL}^{-1}, 12 \mu \mathrm{g} \mathrm{mL}^{-1}, 16 \mu \mathrm{g} \mathrm{mL}^{-1}\right.$, and $\left.20 \mu \mathrm{g} \mathrm{mL}^{-1}\right)$ from M. emarginata leaves was tested on late third instar larvae of Anopheles stephensi, Aedes aegypti and Culex quinquefasciatus [123]. Distilled water and silver nitrate served as controls. It was reported that the aqueous leaf extracts and biosynthesized silver nanoparticles had a larvicidal effect on the mosquitoes in a dose-dependent manner. However, in comparison with the aqueous leaf extract of $M$. emarginata, the biosynthesized silver nanoparticles had higher larvicidal activity against $A$. stephensi, $A$. aegypti and $C$. quinquefasciatus larvae with $\mathrm{LC}_{50}$ values of 8.4 $\mu \mathrm{g} \mathrm{mL}{ }^{-1}, 9.2 \mu \mathrm{g} \mathrm{mL}^{-1}$ and $10.0 \mu \mathrm{g} \mathrm{mL}^{-1}$, respectively at $20 \mu \mathrm{g} \mathrm{mL}^{-1}$. The M. emarginata biosynthesized silver nanoparticles were reported to be safer to non-target aquatic biocontrol agents (Anisops bouvieri, Diplonychus indicus and Gambusia affinis), with $\mathrm{LC}_{50}$ range of $416 \mu \mathrm{g} \mathrm{mL}^{-1}$ to $25,154 \mu \mathrm{g} \mathrm{mL}^{-1}$. It was suggested that the silver nanoparticles enhanced the leaf extracts' bioactivity. The authors attributed the larvicidal activity to the silver 
nanoparticles and the mosquitoes' larvae extracellular lipoprotein matrix interaction, increasing cell plasma membrane permeability. They further reported that the interaction(s) between the silver nanoparticles and phosphorous- or sulfur-containing compounds might have caused enzymes and organelles denaturation, thereby reducing ATP synthesis, which leads to the loss of cellular function and death. The low toxicity effect of $M$. emarginata silver nanoparticles on non-target organisms was suggested to be partially due to the bigger body size of organisms compared with mosquito instars.

A study was carried out by Santos et al. [80] on the control of coffee plants pest, Leucoptera coffeella, by oviposition reduction using methanol leaf extracts of 19 plants, including $M$. tomentosa $\left(8.9 \mathrm{mg} \mathrm{mL}^{-1}\right)$. The authors also assessed the effect of three isolated compounds (cis-tiliroside (2), trans-tiliroside, and ursolic acid (59)) from $M$. tomentosa leaves; Tween 80 and chlorpyrifos served as negative and positive controls. It was reported that only $M$. tomentosa methanol extract, ursolic acid (59) and cis-tiliroside (2) were effective in reducing L. coffeella oviposition on coffee plant leaves with oviposition reduction of $6 \%, 0 \%$ and $11 \%$, respectively like the control chlorpyrifos $(0 \%)$. Two of the isolated bioactive compounds (ursolic acid (59) and cis-tiliroside (2)) from $M$. tomentosa leaf extract were suggested to be responsible for the oviposition reduction by inhibiting glycogen phosphorylases (by binding to the pest's allosteric site) and xanthine dehydrogenases, respectively. The results suggest a possible use of Merremia species extract in the production of green insecticides and repellents to control agricultural pests and mosquito vectors.

Table 6. Summary of the biological activities of Merremia species.

\begin{tabular}{|c|c|c|c|c|c|c|c|}
\hline Species & $\begin{array}{l}\text { Biological } \\
\text { Activity }\end{array}$ & Plant Part & Extract & $\begin{array}{c}\text { Concentration or } \\
\text { Dose }\end{array}$ & Model & Result & Reference \\
\hline \multirow[t]{2}{*}{ M. aegyptia } & $\begin{array}{l}\text { Antioxidant } \\
\text { activity }\end{array}$ & Seeds & Methanol & $500 \mu \mathrm{g} \mathrm{mL}^{-1}$ & $\begin{array}{l}\text { In vitro: DPPH } \\
\text { assay; ascorbic } \\
\text { acid was used as } \\
\text { a standard }\end{array}$ & $\begin{array}{l}\text { Extract had } 90 \% \\
\left(\mathrm{IC}_{50} \text { values of }\right. \\
84) \text { free radical } \\
\text { scavenging effect }\end{array}$ & [117] \\
\hline & $\begin{array}{l}\text { Insecticidal } \\
\text { activity }\end{array}$ & Leaves & $\begin{array}{l}\text { Acetone and its } \\
\text { hexane fraction }\end{array}$ & $250 \mu \mathrm{g} \mathrm{mL}^{-1}$ & $\begin{array}{c}\text { In vivo: } \\
\text { larvicidal } \\
\text { activity against } \\
\text { Aedes aegypti } \\
\text { fourth instar } \\
\text { larvae; } \\
\text { Temephos } \\
\text { served as a } \\
\text { standard }\end{array}$ & $\begin{array}{c}\text { Extracts } \\
\text { exhibited over } \\
100 \% \text { activity } \\
\text { (with } \mathrm{LD}_{50} \\
\text { values of } 120.7 \\
\mu \mathrm{g} \mathrm{mL} \mathrm{m}^{-1} \text { and } \\
144.3 \mu \mathrm{g} \mathrm{mL}^{-1} \\
\text { respectively) }\end{array}$ & [122] \\
\hline \multirow[t]{2}{*}{ M. borneensis } & Antifungal & Leaves & Methanol & $60-400 \mathrm{mg} \mathrm{mL}^{-1}$ & $\begin{array}{c}\text { In vitro: } \\
\text { antifungal } \\
\text { activity against a } \\
\text { mold strain }(A . \\
\text { brasiliensis) and } \\
\text { two yeast strains } \\
\text { (Candida albicans } \\
\text { and } S \text {. cerevisiae) } \\
\text { using agar well } \\
\text { diffusion method }\end{array}$ & $\begin{array}{l}\text { Extract showed } \\
\text { concentration- } \\
\text { dependent } \\
\text { activity } \\
\text { (inhibition zones } \\
\text { range: } 5.5-15.5 \\
\text { mm) }\end{array}$ & [63] \\
\hline & $\begin{array}{l}\text { Antioxidant } \\
\text { activity }\end{array}$ & Leaves, stems & Aqueous ethanol & $100 \mu \mathrm{g} \mathrm{mL}^{-1}$ & $\begin{array}{c}\text { In vitro: } \\
\text { phosphomolyb- } \\
\text { denum method, } \\
\beta \text {-carotene- } \\
\text { linoleate model } \\
\text { system and } \\
\text { DPPH assays; } \\
\text { ascorbic acid and } \\
\text { BHA served as } \\
\text { references }\end{array}$ & $\begin{array}{l}\text { Phosphomolybdenum } \\
\text { method: } 31 \% \\
\text { antioxidant } \\
\text { capacity, } \\
\beta \text {-carotene } \\
\text { bleaching: } 84 \% \\
\text { inhibition, } \\
\text { DPPH: } 80 \% \\
\text { radical } \\
\text { scavenging }\end{array}$ & [22] \\
\hline M. dissecta & Antibacterial & Leaves, flowers & $\begin{array}{l}\text { Diethyl ether, } \\
\text { methanol }\end{array}$ & $100 \mu \mathrm{g} \mathrm{mL}^{-1}$ & $\begin{array}{c}\text { In vitro: } \\
\text { antibacterial } \\
\text { activity against } S \text {. } \\
\text { aureus, } P \text {. } \\
\text { aeruginosa by } \\
\text { biofilm } \\
\text { inhibition; } \\
\text { quorum sensing } \\
\text { inhibitor } \\
\text { (azithromycin) } \\
\text { served as a } \\
\text { control }\end{array}$ & $\begin{array}{c}\text { Extracts } \\
\text { decreased the } \\
\text { biosynthesis of } \\
\text { N-acyl } \\
\text { homoserine } \\
\text { lactone by } 72 \% \text {, } \\
\text { attenuated the } \\
\text { expression of } \\
\text { elastase activity } \\
\text { ( } 27 \% \text { ) and } \\
\text { biofilm } \\
\text { production (55\%) } \\
\text { in P. aeruginosa }\end{array}$ & [4] \\
\hline
\end{tabular}


Table 6. Cont.

\begin{tabular}{|c|c|c|c|c|c|c|c|}
\hline Species & $\begin{array}{l}\text { Biological } \\
\text { Activity }\end{array}$ & Plant Part & Extract & $\begin{array}{l}\text { Concentration } \\
\text { or Dose }\end{array}$ & Model & Result & Reference \\
\hline & $\begin{array}{l}\text { Antioxidant } \\
\text { activity }\end{array}$ & $\begin{array}{l}\text { Leaves, seeds, } \\
\text { stems }\end{array}$ & Methanol & $500 \mu \mathrm{g} \mathrm{mL}^{-1}$ & $\begin{array}{l}\text { In vitro: DPPH assay; } \\
\text { ascorbic acid was used } \\
\text { as a standard }\end{array}$ & $\begin{array}{c}\text { Leaves, seeds and stems } \\
\text { extracts exhibited } 75 \% \text {, } \\
90 \% \text {, and } 93 \% \text {, free } \\
\text { radical scavenging with } \\
\mathrm{IC}_{50} \text { of } 82,80 \text {, and } 61 \mu \mathrm{g} \\
\mathrm{mL}^{-1} \text {, respectively }\end{array}$ & [117] \\
\hline \multirow[t]{5}{*}{ M. emarginata } & $\begin{array}{l}\text { Cancer cell } \\
\text { cytotoxicity }\end{array}$ & Whole plant & Ethyl acetate & $50 \mu \mathrm{g} \mathrm{mL}^{-1}$ & $\begin{array}{l}\text { In vitro: human cancer } \\
\text { cell lines (prostate } \\
\text { carcinoma (DU-145); } \\
\text { Lung carcinoma (A549), } \\
\text { mouth carcinoma (KB) } \\
\text { and pancreas carcinoma } \\
\text { (MIA-PaCa-2)) using } \\
\text { the 3-(4,5- } \\
\text { Dimethylthiazol-2-yl)- } \\
\text { 2,5-diphenyltetrazolium } \\
\text { bromide (MTT) } \\
\text { reduction assay }\end{array}$ & $\begin{array}{l}\text { Ethyl acetate extract } \\
\text { was the most effective, } \\
\text { inhibiting the } \\
\text { proliferation of cell lines } \\
\text { A549, KB, MIA-PaCa-2, } \\
\text { and DU-145 with the } \\
\mathrm{IC}_{50} \text { values of } 28.5 \mu \mathrm{g} \\
\mathrm{mL}^{-1}, 37.2 \mu \mathrm{g} \mathrm{mL}-1,51 \\
\mu \mathrm{g} \mathrm{mL}^{-1} \text {, and } 69.4 \mu \mathrm{g} \\
\mathrm{mL}^{-1} \text {, respectively }\end{array}$ & [3] \\
\hline & $\begin{array}{l}\text { Cancer cell } \\
\text { cytotoxicity }\end{array}$ & Leaves & Hexane & $25 \mu \mathrm{g} \mathrm{mL}^{-1}$ & $\begin{array}{l}\text { In vitro: human cancer } \\
\text { cell lines (lung (A549), } \\
\text { breast (MCF-7), } \\
\text { stomach (AGS), colon } \\
\text { (COLO } 320 \text { DM)) and } \\
\text { monkey normal kidney } \\
\text { epithelial cells (VERO) }\end{array}$ & $\begin{array}{c}\text { Extract inhibited A549 } \\
\text { and COLO } 320 \text { DM cell } \\
\text { line proliferation (with } \\
\mathrm{IC}_{50} \text { values of } 15.5 \mu \mathrm{g} \\
\mathrm{mL}^{-1} \text { and } 18.4 \mu \mathrm{g} \\
\mathrm{mL}^{-1} \text {, respectively) and } \\
\text { had minimal toxicity } \\
\left(\mathrm{IC}_{50} \text { value of } 65.2 \mu \mathrm{g}\right. \\
\mathrm{mL}^{-1} \text { ) for VERO }\end{array}$ & [88] \\
\hline & Anti-diabetic & Whole plant & Methanol & $\begin{array}{l}100,200, \mathrm{and} \\
400 \mathrm{mg} \mathrm{kg}^{-1}\end{array}$ & $\begin{array}{c}\text { In vivo: } \\
\text { streptozotocin-induced } \\
\text { (intraperitoneally) } \\
\text { diabetic male Wistar } \\
\text { rats; assessment of } \\
\text { blood glucose levels, } \\
\text { plasma insulin and } \\
\text { body weight, } \\
\text { glycosylated } \\
\text { hemoglobin, total } \\
\text { hemoglobin, serum } \\
\text { creatinine, serum urea, } \\
\text { hexokinase, fructose-1, } \\
\text { 6-bisphosphatase and } \\
\text { glucose-6-phosphatase, } \\
\text { total protein and } \\
\text { pancreatic tissue } \\
\text { histology }\end{array}$ & $\begin{array}{l}\text { All extract doses } \\
\text { lowered blood glucose } \\
\text { levels to values } \\
\text { comparable to standard } \\
\text { drug (glibenclamide) } \\
\text { and restored plasma } \\
\text { insulin, body weight, } \\
\text { glycosylated } \\
\text { hemoglobin, total } \\
\text { hemoglobin, serum } \\
\text { creatinine, serum urea, } \\
\text { hexokinase, fructose-1, } \\
\text { 6-bisphosphatase, and } \\
\text { glucose-6-phosphatase, } \\
\text { and total protein to } \\
\text { levels near normal } \\
\text { control (sodium } \\
\text { chloride). In the } \\
\text { histological assessment, } \\
\text { the extracts (100 and } 200 \\
\text { mg kg }{ }^{-1}, \text { respectively) } \\
\text { showed mild and } \\
\text { moderate expansion of } \\
\text { the diabetic rats' } \\
\text { pancreatic islets, while } \\
\text { the } 400 \text { mg kg }{ }^{-1} \text { extract } \\
\text { showed prominent } \\
\text { hyperplastic islet } \\
\text { similar to that of the } \\
\text { glibenclamide-treated } \\
\text { group }\end{array}$ & [93] \\
\hline & Antibacterial & Leaves & $\begin{array}{l}\text { Aqueous, } \\
\text { petroleum } \\
\text { ether, and } \\
\text { methanol }\end{array}$ & $\begin{array}{l}10 \mu \mathrm{g} \mathrm{mL}^{-1} \\
25 \mu \mathrm{g} \mathrm{mL}^{-1}, \\
50 \mu \mathrm{g} \mathrm{mL}^{-1} \\
100 \mu \mathrm{g} \mathrm{mL}^{-1}\end{array}$ & $\begin{array}{l}\text { In vitro: antibacterial } \\
\text { activity against } B \text {. cereus, } \\
\text { E. coli, } P \text {. aeruginosa, and } \\
\text { S. aureus by disk } \\
\text { diffusion assay; } \\
\text { penicillin standard drug }\end{array}$ & $\begin{array}{l}\text { All solvent extracts } \\
\text { were effective against } \\
\text { all the bacteria (zone of } \\
\text { inhibition range: } \\
0.7-13.7 \mathrm{~mm} \text { ) compared } \\
\text { with penicillin } \\
(16.0-17.0 \mathrm{~mm})\end{array}$ & [95] \\
\hline & Antibacterial & Leaves & $\begin{array}{l}\text { Aqueous, } \\
\text { acetone, } \\
\text { chloroform, } \\
\text { petroleum } \\
\text { ether }\end{array}$ & $200 \mathrm{mg} \mathrm{mL}^{-1}$ & $\begin{array}{l}\text { In vitro: antibacterial } \\
\text { activity against seven } \\
\text { bacteria by paper disk } \\
\text { diffusion; Ciprofloxacin } \\
\text { as a reference drug }\end{array}$ & $\begin{array}{l}\text { All solvent extracts } \\
\text { were effective against } \\
\text { five bacteria, } \\
\text { particularly } L \text {. } \\
\text { rhamnosus ( } 20 \% \text { zone of } \\
\text { inhibition each); the } \\
\text { most effective (acetone } \\
\text { extract) had maximum } \\
\text { inhibitory ( } 24 \% \text { ) against } \\
\text { S. epidermidis }\end{array}$ & [96] \\
\hline
\end{tabular}


Table 6. Cont.

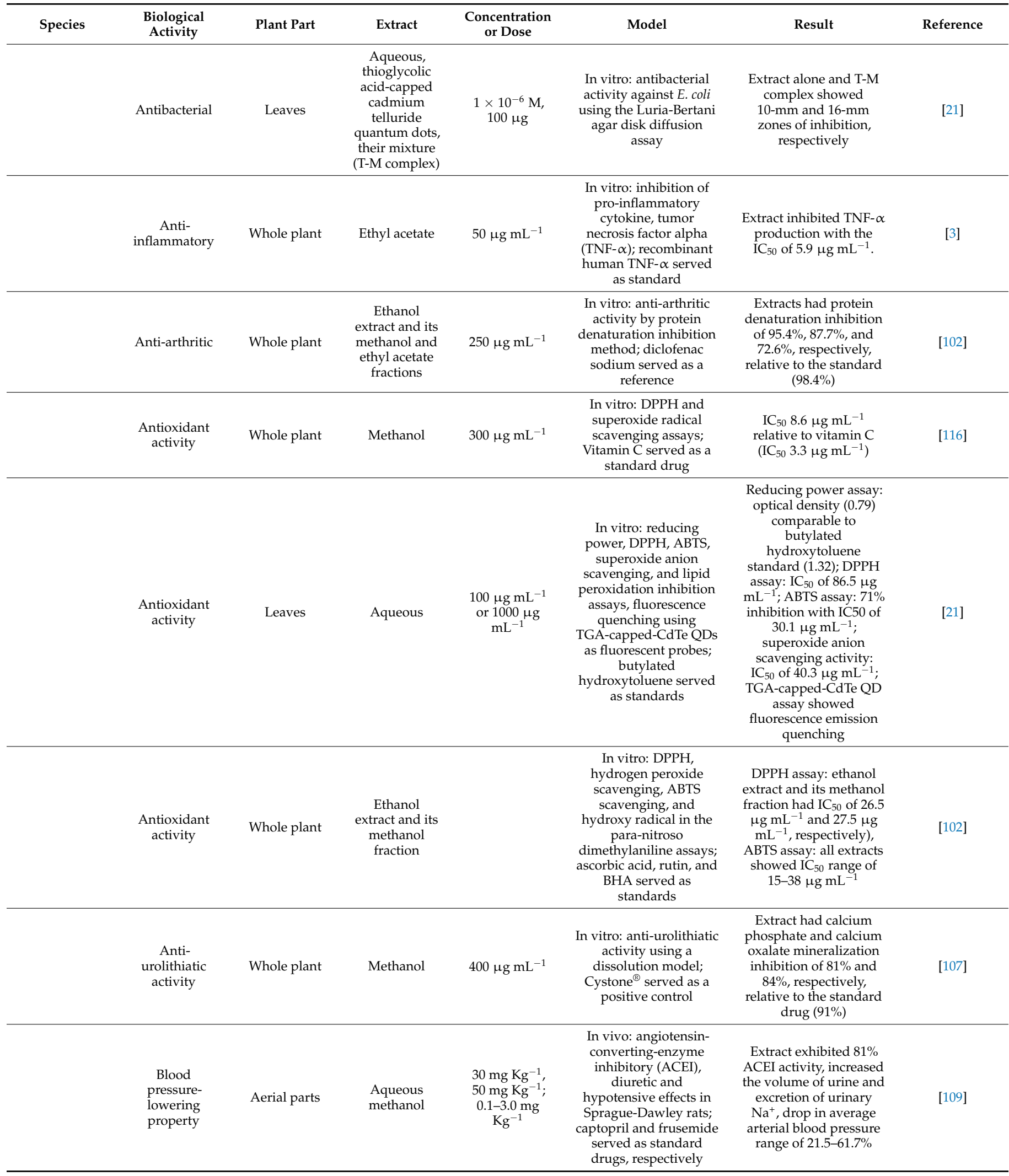


Table 6. Cont.

\begin{tabular}{|c|c|c|c|c|c|c|c|}
\hline Species & $\begin{array}{l}\text { Biological } \\
\text { Activity }\end{array}$ & Plant Part & Extract & $\begin{array}{l}\text { Concentration } \\
\text { or Dose }\end{array}$ & Model & Result & Reference \\
\hline & $\begin{array}{l}\text { Insecticidal } \\
\text { activity }\end{array}$ & Leaves & $\begin{array}{c}\text { Leaf silver } \\
\text { nanoparticles }\end{array}$ & $20 \mu \mathrm{g} \mathrm{mL}^{-1}$ & $\begin{array}{c}\text { In vivo: larvicidal } \\
\text { activity against late } \\
\text { third instar larvae of } A \text {. } \\
\text { stephensi, } A \text {. aegypti and } \\
\text { C. quinquefasciatus; } \\
\text { distilled water and } \\
\text { silver nitrate served as a } \\
\text { control }\end{array}$ & $\begin{array}{l}\text { Larvicidal activity on } A \text {. } \\
\text { stephensi, A. aegypti and } \\
\text { C. quinquefasciatus } \\
\text { larvae with } \mathrm{LC}_{50} \text { values } \\
\text { of } 8.4 \mu \mathrm{g} \mathrm{mL}-1,9.2 \mu \mathrm{g} \\
\mathrm{mL}^{-1} \text {, and } 10.0 \mu \mathrm{g} \\
\mathrm{mL}^{-1} \text {, respectively }\end{array}$ & [123] \\
\hline & $\begin{array}{l}\text { Nephroprotective } \\
\text { activity }\end{array}$ & Leaves & Ethanol & $250 \mathrm{mg} \mathrm{kg}^{-1}$ & $\begin{array}{c}\text { In vivo: } \\
\text { nephroprotective } \\
\text { activity against } \\
\text { gentamicin-induced } \\
\text { (intraperitoneally) } \\
\text { nephrotoxicity in adult } \\
\text { albino Wistar rats using } \\
\text { a histopathology } \\
\text { examination }\end{array}$ & $\begin{array}{l}\text { Extract caused } \\
\text { regeneration of } \\
\text { glomerular, tubular, and } \\
\text { proximal tubular } \\
\text { epithelial cells of } \\
\text { damaged kidney }\end{array}$ & [64] \\
\hline M. hederacea & $\begin{array}{l}\text { Multidrug } \\
\text { resistance } \\
\text { reversal } \\
\text { activity }\end{array}$ & Aerial parts & $\begin{array}{c}\text { Isolated } \\
\text { compounds } \\
\text { (merremins } \\
\text { A-G and } \\
\text { murucoidin IV, } \\
\text { murucoidin V, } \\
\text { stoloniferin IV, } \\
\text { and } \\
\text { murucoidin } \\
\text { XVII) }\end{array}$ & $25 \mu \mathrm{M}$ & $\begin{array}{c}\text { In vivo: multidrug } \\
\text { resistance reversal } \\
\text { activity in KB/VCR cell } \\
\text { lines using a } \\
\text { sulforhodamine B assay; } \\
\text { vinblastine served as a } \\
\text { reference }\end{array}$ & $\begin{array}{c}\text { Noncytotoxic inhibition } \\
\text { ratios less than } 50 \% \\
(0.91 \%,-0.08 \% .0 .23 \% \text {. } \\
7.73 \% \text {, respectively at } 25 \\
\mu \mathrm{M}) \text { for compounds } \mathrm{A}, \\
\mathrm{E}, \mathrm{F} \text {, and murucoidin V } \\
\text { with } \mathrm{IC}_{50} \text { values of } \\
0.253,0.036,0.230,0.004 \\
\text { and } 0.570\end{array}$ & [74] \\
\hline \multirow[t]{4}{*}{ M. mammosa } & Anti-diabetic & Whole plant & $\begin{array}{l}\text { Hexane, ethyl } \\
\text { acetate, } \\
\text { methanol }\end{array}$ & $\begin{array}{c}25 \mu \mathrm{g} \text { GAE } \\
\mathrm{mL}^{-1}, 100 \mu \mathrm{L}\end{array}$ & $\begin{array}{l}\text { In vitro: } \alpha \text {-amylase and } \\
\alpha \text {-glucosidase } \\
\text { inhibition }\end{array}$ & $\begin{array}{c}\text { Extracts exhibited } \\
\alpha \text {-amylase inhibition } \\
(48 \%, 43 \% \text {, and } 12 \% \text {, } \\
\text { respectively) and } \\
\alpha \text {-glucosidase } \\
\text { inhibition }(66 \%, 52 \%, \\
\text { and } 13 \% \text {, respectively) }\end{array}$ & [56] \\
\hline & Anti-influenza & Tuber & $\begin{array}{l}\text { Ethyl acetate } \\
\text { fraction of } \\
\text { methanol } \\
\text { extract }\end{array}$ & $1000 \mu \mathrm{g} \mathrm{mL}^{-1}$ & $\begin{array}{l}\text { In vivo: anti-influenza } \\
\text { A (subtype H1N1) } \\
\text { activity using } \\
\text { hemagglutinin assay; } \\
\text { Zanamivir served as } \\
\text { positive control }\end{array}$ & $\begin{array}{l}\text { Extract had a strong } \\
\text { anti-influenza effect } \\
\text { similar to Zanamivir, } \\
\text { reducing hemagglutinin } \\
\text { virus titer by } 94 \% \text { and } \\
100 \% \text {, respectively, at } \\
1000 \mu \mathrm{g} \mathrm{mL}^{-1}\end{array}$ & [99] \\
\hline & $\begin{array}{l}\text { Antioxidant } \\
\text { activity }\end{array}$ & Whole plant & Methanol & $2 \underset{\mathrm{mL}^{-1}}{2 \mathrm{mg} \text { GAE }}$ & $\begin{array}{l}\text { In vitro: DPPH, } \\
\text { hydroxyl radical, and } \\
\text { superoxide anion } \\
\text { scavenging; methanol } \\
\text { and vitamin C served as } \\
\text { negative and positive } \\
\text { controls, respectively }\end{array}$ & $\begin{array}{c}\text { Methanol extract } \\
\text { showed } \mathrm{IC}_{50} \text { value of } \\
0.7 \mathrm{GAE} \mathrm{ml} \mathrm{H}^{-1} \text { relative to } \\
\text { the positive control }(0.9 \\
\left.\mathrm{GAE} \mathrm{ml}^{-1}\right) \text { in the DPPH } \\
\text { assay, } 92 \% \text { inhibition in } \\
\text { the hydroxyl radical } \\
\text { scavenging assays, } 25 \% \\
\text { inhibition in the } \\
\text { superoxide anion assay }\end{array}$ & [56] \\
\hline & $\begin{array}{l}\text { Wound- } \\
\text { healing } \\
\text { property }\end{array}$ & Whole plant & $\begin{array}{c}\text { Ethyl acetate, } \\
\mathrm{n} \text {-hexane, and } \\
\text { water fractions } \\
\text { of ethanol }\end{array}$ & $25 \mathrm{mg} \mathrm{kg}^{-1}$ & $\begin{array}{l}\text { In vivo: wound-healing } \\
\text { effect in } \\
\text { streptozotocin-induced } \\
\text { (intraperitoneally) } \\
\text { diabetic male Winstar } \\
\text { rats; wound was } \\
\text { excised using the } \\
\text { Morton method; } \\
\text { aquadest and } \\
\text { gentamicin served as } \\
\text { negative and positive } \\
\text { controls, respectively }\end{array}$ & $\begin{array}{c}\text { Extracts showed wound } \\
\text { diameter }(72 \%, 62 \% \text {, } \\
\text { and } 54 \% \text {, respectively) } \\
\text { compared with the } \\
\text { negative control (114 } \\
\text { mm), and percentage } \\
\text { wound reduction }(89 \% \text {, } \\
90 \% \text {, and } 93 \% \text {, } \\
\text { respectively) similar to } \\
\text { the positive control } \\
(92 \%)\end{array}$ & [112] \\
\hline
\end{tabular}


Table 6. Cont.

\begin{tabular}{|c|c|c|c|c|c|c|c|}
\hline Species & $\begin{array}{l}\text { Biological } \\
\text { Activity }\end{array}$ & Plant Part & Extract & $\begin{array}{l}\text { Concentration } \\
\text { or Dose }\end{array}$ & Model & Result & Reference \\
\hline & $\begin{array}{l}\text { Wound- } \\
\text { healing } \\
\text { property }\end{array}$ & Whole plant & $\begin{array}{c}\text { Gel } \\
\text { formulations } \\
\text { (hydrox- } \\
\text { ypropyl- } \\
\text { methylcellu- } \\
\text { lose (HPMC), } \\
\text { Carbopol or } \\
\text { sodium car- } \\
\text { boxymethyl- } \\
\text { cellulose (Na } \\
\text { CMC) in 10\% } \\
\text { water fraction) } \\
\text { of ethanol } \\
\text { extract }\end{array}$ & $\begin{array}{l}1.5 \% \text { gelling } \\
\text { agent }\end{array}$ & $\begin{array}{l}\text { In vivo: vascular } \\
\text { endothelial growth } \\
\text { factor, hydroxyproline } \\
\text { levels, and collagen } \\
\text { density assessments in } \\
\text { streptozotocin-induced } \\
\text { (intraperitoneally) } \\
\text { diabetic Winstar rats; } \\
\text { wound was excised } \\
\text { using the Morton } \\
\text { method; distilled water } \\
\text { (negative control), } \\
\text { neomycin sulfate, and } \\
\text { placenta extract gel } \\
\text { (positive control) }\end{array}$ & $\begin{array}{l}\text { Extracts had similar } \\
\text { healing effect relative to } \\
\text { the positive control in } \\
\text { terms of optimum level } \\
\text { vascular endothelial } \\
\text { growth factor } \\
\text { expression, } \\
\text { hydroxyproline levels, } \\
\text { and collagen density }\end{array}$ & [57] \\
\hline \multirow[t]{3}{*}{ M. peltata } & Anti-diabetic & $\begin{array}{l}\text { Leaves and } \\
\text { stem }\end{array}$ & $\begin{array}{l}\text { Hexane, ethyl } \\
\text { acetate, and } \\
\text { methanol }\end{array}$ & $\begin{array}{c}20-100 \mu \mathrm{g} \\
\mathrm{mL}^{-1}\end{array}$ & $\begin{array}{c}\text { In vitro: alpha } \\
\text { glucosidase enzyme } \\
\text { inhibition }\end{array}$ & $\begin{array}{l}\text { Stem methanolic extract } \\
\text { had the best activity } \\
\text { with } \mathrm{IC}_{50} \text { value } 47.44 \\
\mu \mathrm{g} / \mathrm{mL} \text {, almost two } \\
\text { times better than } \\
\text { acarbose as a positive } \\
\text { control }\left(\mathrm{IC}_{50}=98.38\right. \\
\mu \mathrm{g} / \mathrm{mL}) \text {. Leaves } \\
\text { methanolic extract, } \\
\text { leaves ethyl acetate } \\
\text { extract, and stem ethyl } \\
\text { acetate extract also give } \\
\text { better activity of alpha } \\
\text { glucosidase inhibitors } \\
\text { than acarbose with } \mathrm{IC}_{50} \\
\text { value } 67.24 \mu \mathrm{g} / \mathrm{mL}, \\
69.38 \mu \mathrm{g} / \mathrm{mL}, \text { and } 72.85 \\
\mu \mathrm{g} / \mathrm{mL}, \text { respectively. }\end{array}$ & [90] \\
\hline & Antibacterial & Leaves & Ethanol & $5-20 \mu \mathrm{g} \mathrm{mL}^{-1}$ & $\begin{array}{l}\text { In vitro: antibacterial } \\
\text { activity against } B \text {. } \\
\text { subtilis, } S \text {. aureus, } P \text {. } \\
\text { aeruginosa, E. coli using } \\
\text { the Kirby-Bauer disk } \\
\text { diffusion method; } \\
\text { streptomycin and } \\
\text { chloramphenicol served } \\
\text { as positive controls }\end{array}$ & $\begin{array}{l}\text { Extract showed } 5.7-\mathrm{mm} \\
\text { average zone of } \\
\text { inhibition against } B \text {. } \\
\text { subtilis and } S \text {. aureus (at } \\
10 \mu \mathrm{g} \mathrm{mL}^{-1} \text { and } 20 \mu \mathrm{g} \\
\mathrm{mL}^{-1} \text {, respectively), } \\
4.7-\mathrm{mm} \text { and } 2.7-\mathrm{mm} \\
\text { average zones of } \\
\text { inhibition against } P \text {. } \\
\text { aeruginosa and E. coli (at } \\
15 \mu \mathrm{g} \mathrm{mL}^{-1} \text { and } 5 \mu \mathrm{g} \\
\mathrm{mL}^{-1} \text {, respectively) }\end{array}$ & [19] \\
\hline & Anti-diabetic & $\begin{array}{l}\text { Leaves and } \\
\text { stem }\end{array}$ & $\begin{array}{l}\text { Hexane, ethyl } \\
\text { acetate, and } \\
\text { methanol }\end{array}$ & $\begin{array}{c}20-100 \mu \mathrm{g} \\
\mathrm{mL}^{-1}\end{array}$ & $\begin{array}{l}\text { In vitro: DPPH and } \\
\text { FRAP }\end{array}$ & $\begin{array}{c}\text { Stem methanolic extract } \\
\text { had the highest } \\
\text { antioxidant activity } \\
\text { with } \mathrm{IC}_{50} \text { value of } 47.41 \\
\mu \mathrm{g} / \mathrm{mL} \text { in DPPH and } \\
\text { total antioxidant power } \\
\text { of } 340.04 \mu \mathrm{mol} / \mathrm{g} \text { in } \\
\text { FRAP. }\end{array}$ & [90] \\
\hline M. tomentosa & $\begin{array}{l}\text { Insecticidal } \\
\text { activity }\end{array}$ & Leaves & $\begin{array}{l}\text { Methanol and } \\
\text { isolated } \\
\text { compounds } \\
\text { (ursolic acid } \\
\text { and } \\
\text { cis-tiliroside) }\end{array}$ & $8.9 \mathrm{mg} \mathrm{mL}^{-1}$ & $\begin{array}{l}\text { In vivo: oviposition } \\
\text { reduction in L. coffeella; } \\
\text { chlorpyrifos serves as } \\
\text { positive control }\end{array}$ & $\begin{array}{c}\text { Extract and isolated } \\
\text { compounds reduced } L \text {. } \\
\text { coffeella oviposition to } \\
6 \%, 0 \% \text {, and } 11 \% \text {, } \\
\text { respectively) relative to } \\
\text { the control chlorpyrifos } \\
(0 \%)\end{array}$ & [80] \\
\hline
\end{tabular}


Table 6. Cont.

\begin{tabular}{|c|c|c|c|c|c|c|c|}
\hline Species & $\begin{array}{l}\text { Biological } \\
\text { Activity }\end{array}$ & Plant Part & Extract & $\begin{array}{l}\text { Concentration } \\
\text { or Dose }\end{array}$ & Model & Result & Reference \\
\hline \multirow[t]{4}{*}{ M. tridentata } & Anti-diabetic & Roots & Aqueous & $\begin{array}{c}50-150 \mathrm{mg} \\
\mathrm{kg}^{-1}\end{array}$ & $\begin{array}{l}\text { In vivo: normoglycemic, } \\
\text { glucose-loaded } \\
\text { hyperglycemic, and } \\
\text { streptozotocin-induced } \\
\text { (intraperitoneally) } \\
\text { diabetic rats; blood } \\
\text { glucose levels, serum } \\
\text { insulin, triglycerides, } \\
\text { total cholesterol, } \\
\text { glycogen (in skeletal } \\
\text { muscle and liver), and } \\
\text { lipid peroxidation in } \\
\text { pancreatic tissue } \\
\text { estimations }\end{array}$ & 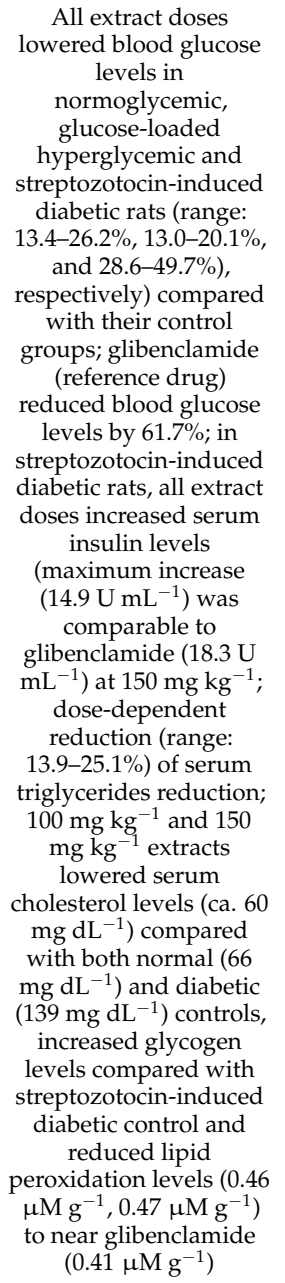 & [52] \\
\hline & Antibacterial & Leaves & Methanol & $\begin{array}{c}0.01 \mathrm{mg} \mathrm{mL}^{-1}, \\
-1.00 \mathrm{mg} \\
\mathrm{mL}^{-1} ; 0.05 \mathrm{mg} \\
\mathrm{mL}^{-1},-1 \mathrm{mg} \\
\mathrm{mL}^{-1}\end{array}$ & $\begin{array}{c}\text { In vitro: antibacterial } \\
\text { activity against } \\
\text { Gram-positive (Bacillus } \\
\text { subtilis and } \\
\text { Staphylococcus aureus) } \\
\text { and Gram-negative } \\
\text { (Escherichia coli and } \\
\text { Vibrio parahaemolyticus) } \\
\text { bacteria using turbidity } \\
\text { and zone formation } \\
\text { methods }\end{array}$ & $\begin{array}{l}\text { Gram-positive MIC } \\
\text { range: } 0.25-0.5 \mathrm{mg} \\
\mathrm{mL}^{-1} \text {; zone formation } \\
\text { range: } 10-14 \mathrm{~mm} ; \\
\text { Gram-negative MIC } \\
\text { range: } 0.5-1 \mathrm{mg} \mathrm{mL}{ }^{-1} \text {; } \\
\text { zone formation range: } \\
9-11 \mathrm{~mm}\end{array}$ & [94] \\
\hline & Antibacterial & Stem & Methanol & $100 \mathrm{mg} \mathrm{mL}^{-1}$ & $\begin{array}{l}\text { In vitro: antibacterial } \\
\text { activity against S. aureus } \\
\text { and B. subtilis, Klebsiella } \\
\text { pneumoniae, E. coli, and } \\
\text { Pseudomonas aeruginosa; } \\
\text { ampicillin and } \\
\text { gentamicin standard } \\
\text { drugs }\end{array}$ & $\begin{array}{l}\text { Extract was effective } \\
\text { against } S \text {. aureus and } B \text {. } \\
\text { subtilis (inhibition } \\
\text { zones: } 12.0 \mathrm{~mm} \text { and } \\
11.3 \mathrm{~mm} \text {; MIC: } 3.13 \mathrm{mg} \\
\mathrm{mL}^{-1} \text { and } 6.25 \mathrm{mg} \\
\mathrm{mL}^{-1} \text {, respectively; } \\
\text { minimum bactericidal } \\
\text { concentration: } 12.5 \mathrm{mg} \\
\mathrm{mL}^{-1} \text { and } 100 \mathrm{mg} \mathrm{mL}^{-1} \text {, } \\
\text { respectively) relative to } \\
\text { both standard drugs (10 } \\
\mu \mathrm{g} \mathrm{mL}^{-1}\end{array}$ & [66] \\
\hline & Antifungal & Leaves & Methanol & $\begin{array}{c}0.01-100 \mathrm{mg} \\
\mathrm{mL}^{-1}\end{array}$ & $\begin{array}{l}\text { In vitro: antifungal } \\
\text { activity against } \\
\text { Aspergillus niger, } \\
\text { Saccharomyces cerevisiae } \\
\text { using turbidity and } \\
\text { zone formation } \\
\text { methods }\end{array}$ & $\begin{array}{l}\text { Extracts were more } \\
\text { effective against } S . \\
\text { cerevisiae (MIC range: } \\
0.25-0.5 \mathrm{mg} \mathrm{mL}^{-1} \text {; zone } \\
\text { formation range: } 10-15 \\
\mathrm{~mm} \text { ). }\end{array}$ & [94] \\
\hline
\end{tabular}


Table 6. Cont.

\begin{tabular}{|c|c|c|c|c|c|c|c|}
\hline Species & $\begin{array}{l}\text { Biological } \\
\text { Activity }\end{array}$ & Plant Part & Extract & $\begin{array}{l}\text { Concentration } \\
\text { or Dose }\end{array}$ & Model & Result & Reference \\
\hline & $\begin{array}{c}\text { Anti- } \\
\text { inflammatory }\end{array}$ & Whole plant & Ethanol & $\begin{array}{l}100 \mathrm{mg} \mathrm{kg}^{-1} \\
200 \mathrm{mg} \mathrm{kg}^{-1}\end{array}$ & $\begin{array}{c}\text { In vivo: } \\
\text { anti-inflammatory } \\
\text { activity on male albino } \\
\text { rats using the } \\
\text { carrageenan-induced } \\
\text { rat paw inflammation } \\
\text { method; indomethacin } \\
\text { served as standard drug }\end{array}$ & $\begin{array}{l}\text { Relative to the standard } \\
\text { drug }(48.5 \%) \text {, extract } \\
\text { was effective with } 38.3 \% \\
\text { and } 42.8 \% \text { inflammation } \\
\text { inhibition at } 100 \mathrm{mg} \\
\mathrm{kg}^{-1} \text { and } 200 \mathrm{mg} \mathrm{kg}^{-1} \\
\text { doses, respectively }\end{array}$ & [55] \\
\hline & $\begin{array}{c}\text { Anti- } \\
\text { inflammatory }\end{array}$ & Roots & $\begin{array}{l}\text { Solvent ether, } \\
\text { ethyl acetate, } \\
\text { butanol and } \\
\text { butanone } \\
\text { fractions of } \\
\text { ethanol extract }\end{array}$ & $300 \mathrm{mg} \mathrm{kg}^{-1}$ & $\begin{array}{l}\text { In vivo: } \\
\text { anti-inflammatory } \\
\text { activity in albino mice } \\
\text { using the } \\
\text { carrageenan-induced } \\
\text { rat paw edema method; } \\
\text { aspirin served as the } \\
\text { standard drug }\end{array}$ & $\begin{array}{c}\text { Extracts were effective } \\
(87 \%, 84 \%, 72 \% \text {, and } \\
68 \% \text {, respectively) in } \\
\text { inhibiting rat paw } \\
\text { edema, relative to the } \\
\text { standard }(59.7 \%)\end{array}$ & [53] \\
\hline & Anti-arthritic & Whole plant & Ethanol & $\begin{array}{l}100 \mathrm{mg} \mathrm{kg}^{-1} \\
\text { and } 200 \mathrm{mg} \\
\mathrm{kg}^{-1}\end{array}$ & $\begin{array}{l}\text { In vivo: anti-arthritic } \\
\text { activity on male albino } \\
\text { rats using the complete } \\
\text { Freund's } \\
\text { adjuvant-induced } \\
\text { arthritis model; } \\
\text { indomethacin served as } \\
\text { the standard drug }\end{array}$ & $\begin{array}{l}\text { Extract had } 49.0 \% \text { and } \\
51.7 \% \text { anti-arthritic } \\
\text { activity at } 100 \mathrm{mg} \mathrm{kg}^{-1} \\
\text { and } 200 \mathrm{mg} \mathrm{kg}^{-1}, \\
\text { respectively, which } \\
\text { were comparable to the } \\
\text { standard drug (55.5\%) }\end{array}$ & [55] \\
\hline & $\begin{array}{l}\text { Antioxidant } \\
\text { activity }\end{array}$ & $\begin{array}{c}\text { Roots, aerial } \\
\text { parts }\end{array}$ & $\begin{array}{l}\text { Acetone, } \\
\text { chloroform, } \\
\text { methanol, and } \\
\text { hot water }\end{array}$ & $200 \mu \mathrm{g} \mathrm{mL}^{-1}$ & $\begin{array}{l}\text { In vitro: } \mathrm{DPPH} \text { and } \\
\text { ABTS, OH॰, FRAP, } \\
\text { phosphomolybdenum } \\
\text { reduction, } \mathrm{Fe}^{2+} \\
\text { chelation, } \\
\beta \text {-carotene/linoleic } \\
\text { acid peroxidation } \\
\text { inhibition, and } \\
\text { antihemolytic activity } \\
\text { assays }\end{array}$ & $\begin{array}{l}\text { Acetone root extract: } \\
\text { IC }_{50} 26.6 \mu \mathrm{g} \mathrm{mL}^{-1} \mathrm{in} \\
\text { DPPH assay; highest } \\
\text { equivalent trolox, FRAP } \\
\text { and } \\
\text { phosphomolybdenum } \\
\text { reduction values } \\
\left(26,270.8 \mu \mathrm{mol} \mathrm{g}^{-1}\right. \\
\text { extract, } 2656.7 \mathrm{mmol}^{-1} \mathrm{Fe} \\
\text { (II) } \mathrm{mg}^{-1} \text { extract and } \\
56.7 \mathrm{~g} \text { ascorbic acid } / 100 \\
\text { g extract, respectively); } \\
\text { root and aerial parts } \\
\text { extracts: OH} \\
\text { scavenging activity } \\
\text { range of } 29.6-59.3 \% \text { and } \\
34.8-52.9 \% ; \text { hot water } \\
\text { extract showed activity } \\
\text { of } 8.0 \text { mg EDTA g }{ }^{-1} \\
\text { extracts in the Fe }{ }^{2+} \\
\text { chelation assay; } \\
\text { inhibition of } \beta \text {-carotene } \\
\text { bleaching (20.7-36.1\% } \\
\text { by the root extract and } \\
13.3-32.9 \% \text { by the aerial } \\
\text { parts extract); acetone } \\
\text { extracts (aerial parts } \\
\text { and roots) and } \\
\text { methanol root extract } \\
\text { exhibited activities } \\
\text { (32.9\%, } 36.1 \% \text {, and } \\
31.8 \%, \text { respectively) } \\
\text { comparable with } \\
\text { BHA standard ( } 36.6 \%) ; \\
\text { higher peroxidation } \\
\text { inhibition activity by all } \\
\text { extracts compared with } \\
\alpha \text {-tocopherol standard; } \\
\text { acetone root extract: } \\
82.7 \% \text { red blood cell } \\
\text { hemolysis inhibition }\end{array}$ & [54] \\
\hline
\end{tabular}


Table 6. Cont.

\begin{tabular}{|c|c|c|c|c|c|c|c|}
\hline Species & $\begin{array}{l}\text { Biological } \\
\text { Activity }\end{array}$ & Plant Part & Extract & $\begin{array}{l}\text { Concentration } \\
\text { or Dose }\end{array}$ & Model & Result & Reference \\
\hline & $\begin{array}{l}\text { Wound- } \\
\text { healing } \\
\text { property }\end{array}$ & Roots & $\begin{array}{l}\text { Solvent ether, } \\
\text { petroleum } \\
\text { ether, ethyl } \\
\text { acetate, } \\
\text { butanol, and } \\
\text { butanone } \\
\text { fractions of } \\
\text { ethanol }\end{array}$ & $\begin{array}{c}250 \mathrm{mg} \mathrm{kg}^{-1} \\
\text { or } 300 \mathrm{mg} \\
\mathrm{kg}^{-1}\end{array}$ & $\begin{array}{l}\text { In vivo: wound-healing } \\
\text { effect in albino mice } \\
\text { using the tensile } \\
\text { strength of wound } \\
\text { models (re-sutured } \\
\text { incision and grass pith } \\
\text { granuloma); Tween } 80 \\
\text { solution served as the } \\
\text { control }\end{array}$ & $\begin{array}{l}\text { Extracts exhibited } \\
\text { higher tensile strength } \\
(215.4 \mathrm{~g}, 213 \mathrm{~g}, 243.7 \mathrm{~g} \text {, } \\
236.1 \mathrm{~g} \text {, and } 232.9 \mathrm{~g} \text {, } \\
\text { respectively) than the } \\
\text { control }(144.3 \mathrm{~g}) \text { in the } \\
\text { re-sutured incision } \\
\text { model; }(221.7 \mathrm{~g}, 210 \mathrm{~g} \text {, } \\
264.1 \mathrm{~g}, 332.8 \mathrm{~g} \text {, and } \\
335.7 \mathrm{~g} \text {, respectively) } \\
\text { than the control (155.4 } \\
\text { g) in the granuloma } \\
\text { model }\end{array}$ & [53] \\
\hline M. umbellata & Antibacterial & Leaves & Ethanol & $1000 \mu \mathrm{g} \mathrm{mL}^{-1}$ & $\begin{array}{l}\text { In vitro: antibacterial } \\
\text { activity against } \\
\text { Klebsiella pneumoniae, S. } \\
\text { aureus, and Pseudomonas } \\
\text { aeruginosa using the } \\
\text { broth microdilution } \\
\text { method; gentamicin } \\
\text { was the positive control }\end{array}$ & $\begin{array}{c}\text { Extract had } 32 \%, 55 \% \text {, } \\
\text { and } 67 \% \text {, respectively, } \\
\text { on the bacterial strains' } \\
\text { growth }\end{array}$ & [62] \\
\hline \multirow[t]{4}{*}{ M. vitifolia } & Anti-arthritic & Leaves & $\begin{array}{l}\text { Aqueous } \\
\text { fraction from } \\
\text { methanol } \\
\text { extract }\end{array}$ & $500 \mu \mathrm{g} \mathrm{mL}^{-1}$ & $\begin{array}{l}\text { In vitro: anti-arthritic } \\
\text { activity by protein } \\
\text { denaturation inhibition } \\
\text { method; diclofenac } \\
\text { sodium served as a } \\
\text { reference }\end{array}$ & $\begin{array}{l}\text { Extracts had protein } \\
\text { denaturation inhibition } \\
\text { of } 64 \% \text { relative the } \\
\text { standard drug }(87 \%)\end{array}$ & [20] \\
\hline & $\begin{array}{l}\text { Anti- } \\
\text { nociceptive } \\
\text { activity }\end{array}$ & Leaves & $\begin{array}{l}\text { Aqueous } \\
\text { fraction from } \\
\text { methanol } \\
\text { extract }\end{array}$ & $\begin{array}{l}200 \mathrm{mg} \mathrm{kg}^{-1} \\
400 \mathrm{mg} \mathrm{kg}^{-1}\end{array}$ & $\begin{array}{c}\text { In vivo: } \\
\text { anti-nociceptive activity } \\
\text { in Swiss Albino mice } \\
\text { using formalin-induced } \\
\text { paw licking (at early } \\
\text { and late stages) and } \\
\text { acetic acid-induced } \\
\text { writhing tests; } \\
\text { diclofenac sodium } \\
\text { (administered } \\
\text { intraperitoneally) } \\
\text { served as a standard } \\
\text { drug }\end{array}$ & $\begin{array}{c}\text { Extract showed } \\
\text { anti-nociceptive activity } \\
(44 \% \text { and } 26 \%, 30 \% \text { and } \\
20 \% \text { in both early and } \\
\text { late test stages, } \\
\text { respectively) relative to } \\
\text { the control ( } 20 \% \text { and } \\
15 \% \text {, respectively) in the } \\
\text { formalin-induced paw } \\
\text { licking test; reduced } \\
(44 \% \text { and } 30 \% \text {, } \\
\text { respectively) abdominal } \\
\text { contortions with } \\
\text { increasing doses of } \\
\text { APFME relative to the } \\
\text { control }(21 \%)\end{array}$ & [20] \\
\hline & $\begin{array}{l}\text { Antioxidant } \\
\text { activity }\end{array}$ & Stems & Ethanol & $120 \mu \mathrm{g} \mathrm{mL}^{-1}$ & $\begin{array}{l}\text { In vitro: DPPH assay; } \\
\text { gallic acid, L-ascorbic } \\
\text { acid, and piperine were } \\
\text { used as references }\end{array}$ & $\mathrm{IC}_{30}$ of $25 \mu \mathrm{g} \mathrm{mL}^{-1}$ & [118] \\
\hline & $\begin{array}{c}\text { Thrombolytic } \\
\text { activity }\end{array}$ & Leaves & $\begin{array}{l}\text { Aqueous } \\
\text { fraction from } \\
\text { methanol } \\
\text { extract }\end{array}$ & $500 \mu \mathrm{g} \mathrm{mL}^{-1}$ & $\begin{array}{l}\text { In vitro: thrombolytic } \\
\text { activity in blood } \\
\text { samples from male and } \\
\text { female (1:1) adult } \\
\text { human volunteers with } \\
\text { no anticoagulant and } \\
\text { oral contraceptive } \\
\text { treatments history; } \\
\text { streptokinase served as } \\
\text { a positive control }\end{array}$ & $\begin{array}{l}\text { Extract exhibited } \\
\text { thrombolytic activity } \\
(42.5 \% \text { clot lysis }) \\
\text { relative to the positive } \\
\text { control }(72.2 \%)\end{array}$ & [20] \\
\hline
\end{tabular}

Results are shown for the most effective doses.

\section{Toxicology}

It is important to evaluate the safety of species in the genus Merremia due to their traditional medicinal usage, and as feed for livestock, even though several species in the genus are non-toxic. However, only a few toxicity studies are available on Merremia species. Acute toxicity study of the ethanolic root extract of $M$. tridentata was performed at dose concentrations of 500 and $1000 \mathrm{mg} / \mathrm{kg}$ body weight. The extract was orally administered to Wistar albino rats and observed for mortality after $72 \mathrm{~h}$. No visible toxicity, behavioral changes, or mortality were observed in rats [124]. In another study, an acute toxicity study of the methanolic whole plant extract of M. emarginata was evaluated in five groups of adult male Wistar rats. The whole plant extract was orally administered to the mice at dose 
levels of 100, 500, 1000, 2000, and $4000 \mathrm{mg} / \mathrm{kg}$ and observed for neurological, behavioral, and autonomic reactions after $24 \mathrm{~h}$ and $72 \mathrm{~h}$. There was no toxic reaction and lethality in the experimental rats at any dose given until the end of the study period [93]. Similarly, Priya et al. [125] reported no toxic effect of the ethanolic leaf extract of M. emarginata at a dose of $2 \mathrm{~g} / \mathrm{kg}$ administered orally to Swiss albino mice after $24 \mathrm{~h}$. A few Merremia species are considered toxic to ruminants. For example, spontaneous poisoning in cattle by $M$. macrocalyx in the Pernambuco state, north-eastern Brazil was reported by Brito et al. [126]. In a recent study, acute and chronic toxicity evaluation of the ethanolic leaf extract of $M$. tridentata was carried out on albino male rats [127]. In the acute toxicity study, animals were orally administered different extract doses of $M$. tridentata $(10,100$, and $1000 \mathrm{mg} / \mathrm{kg}$ b.w.) and monitored for $24 \mathrm{~h}$ for toxicity signs. In the chronic toxicity study, the animals were orally administered different $M$. tridentata extract doses once a day (100, 200, 400 $\mathrm{mg} / \mathrm{kg}$ b.w.) for 14 weeks (100 days). The control group received $0.2 \mathrm{~mL}$ of distilled water. Haematology, serum biochemical indices, and histopathological studies of the kidney, liver, heart, spleen, and lungs of the animals were carried out for the toxic effect of the extract. The results of the acute toxicity study revealed that there was no mortality of any of the rats at 10,100, and $1000 \mathrm{mg} / \mathrm{kg}$ b.w. dose while mortality was only recorded at $5000 \mathrm{mg} / \mathrm{kg}$ b.w. dose. The median lethal dose was estimated to be $2200 \mathrm{mg} / \mathrm{kg}$ b.w. In the chronic toxicity study, the results showed that the extract did not cause any alteration in the hematological parameters at all doses when compared with the control. However, the extract at $400 \mathrm{mg} / \mathrm{kg}$ b.w. caused a significant reduction in the lymphocyte. Chronic exposure of the animals to the plant extract at $100 \mathrm{mg} / \mathrm{kg}$ dosage, when tested for biochemical parameters, had no significant difference when compared with the control animal. However, at higher dosages of 200 and $400 \mathrm{mg} / \mathrm{kg}$ for 100 days, there was a significant increase in the serum levels of the biochemical parameters investigated when compared with the control. The abnormal increase in values of the biochemical parameters such as creatine, urea, transaminases, creatine kinase, among others are indications of the potential toxicity of the extract to the kidney, liver, and heart. Further histopathological studies revealed that the extract at $200 \mathrm{mg} / \mathrm{kg}$ did not show any morphological sign of toxicity on histoarchitecture of the liver, spleen, kidney, lung, and heart, but caused hemorrhage and vascular congestion in the heart, kidney, and lungs. Also, the extract at $400 \mathrm{mg} / \mathrm{kg}$ resulted in renal, myocardial damage, fibrosis, and vascular congestion in the lung, spleen, and liver.

Although the few documented experimental toxicology studies revealed that most Merremia species are safe, the Merremia species extract at chronic administration even at a low dosage of $200 \mathrm{mg} / \mathrm{kg}$ b.w. may not be safe as inferred from the recent toxicity study. There is a need for more toxicity studies to be conducted on the different species to establish their safety, especially for modern clinical use.

\section{Discussion and Future Perspective}

The current review presented the research progression in the genus Merremia and summarized knowledge on its nutritional value, ethnomedicinal uses, phytochemistry, biological activities, and toxicity studies.

The bibliometric analysis of the genus Merremia evaluated the global research trends between 1990 and 2020 based on the relevant data retrieved from Web of Science. We found that a high number of research articles (55\%) were published in the last decade (2011-2020). This may be due to the availability of funds majorly because of the wide traditional uses and bioactivities, the advent of research ideas, and sophisticated analytical tools for chemical analysis. Moreover, interest has been rising for natural product research and development across the globe [128]. The utilization of medicinal plants (particularly from commonly used medicinal plants) as alternative sources for identifying bioactive agents that pharmaceutical industries can employ in the preparation of drugs in modern medicine has substantially risen [129]. These suggest that there may be more funding opportunities for Merremia research, which in turn suggests that articles related to the Merremia species are likely to increase in years to come. 
Most of the leading authors on Merremia related research based on continental production were from Asia. This correlates with the fact that most Merremia species are primarily distributed in the warm and tropical regions of Asia [74]. As identified from the bibliometric analysis, the current research on the Merremia species can be grouped into four thematic areas, viz. drug formulation, chemical analysis (including the nutritional value), treatment of diseases, and taxonomy.

Based on the available reports on the nutritional and antinutritional constituents of Merremia species, they contain important nutritional components that are beneficial to humans. Additionally, the Merremia species can be considered good fodder as several evaluated species meet high nutritional quality criteria for animals, and are therefore suitable for inclusion in the diets of livestock. However, the nutritional analysis and toxicity testing of Merremia species are still less researched as inferred from this study. Future investigation on this genus should include more nutritional and toxicity analyses of the species.

Given the wide distribution of Merremia species in the Asia continent, the literature review revealed that members of the genus are mainly used ethnomedicinally in India, China, Indonesia, Malaysia, and the Philippines. Phytochemistry studies have resulted in the isolation of several secondary metabolites, including flavonoids, phenolics, sesquiterpenoid, alkaloids, resin glycosides, among others in the genus. Several pharmacological investigations have been carried out using in vivo and in vitro techniques in the genus.

In terms of current research, several biological activities have been documented from different species of Merremia, including cancer cell cytotoxicity, antidiabetic, antimicrobial, anti-inflammatory, blood pressure-lowering, wound-healing, multi-drug resistance reversal activities, etc., which supports some of the ethnomedicinal uses of Merremia species. For example, leaves of $M$. mammosa and the root of $M$. tridentata have been used in the traditional treatment of diabetes and diabetic ulcer in Indonesia and India. Available studies have attributed the in vitro and in vivo antidiabetic and wound-healing properties in M. mammosa and M. tridentata to flavonoid and glycoside contents of the species. Also, extracts and fractionated extracts of Merremia species revealed that flavonoids are responsible for the anti-inflammatory activities, which supports the ethnomedicinal use of several Merremia species in treating inflammation and rheumatism.

In India, the Philippines, and Colombia, leaves and whole plants of M. peltata, $M$. umbellata and M. emarginata have been used traditionally as antibacterial and antifungal agents. In vitro antimicrobial studies in these species also showed effective antimicrobial activities that were attributed to different phytochemicals (alkaloids, flavonoids, leucoanthocyanidins, steroids, and phenolics) present in the species.

Generally, most of the biological activities investigated in the Merremia species showed relevant and good activities with $\mathrm{IC}_{50}$ values below $100 \mu \mathrm{g} \mathrm{mL}^{-1}$. The end-point criteria set for medicinal plants to be considered as showing relevant bioactivity include; plant extracts with $\mathrm{IC}_{50}$ values below $100 \mu \mathrm{g} \mathrm{mL}-1$ and pure compounds isolated from plants showing activities with $\mathrm{IC}_{50}$ values below $25 \mu \mathrm{M}$ [1].

Several aspects highlighted below need to be considered and investigated further in the genus. (1) So far, only 11 species have been investigated for their bioactivities from a large genus of over 70 species. Based on the reported bioactivities in the investigated species, other uninvestigated species may be viewed as promising plant species with pharmacological importance. Most of the species that have not been investigated are native to Africa. However, Africa lags behind Asia, Australia, North, and South America in terms of research (including medicinal plant research), presumably due to limited funding resources and infrastructure. More funding should be made available to investigate the several under-exploited medicinal plants in Africa, including the Merremia species, as they offer untold bioactivities that are of nutritional and pharmaceutical relevance. Thus, future research should consider in vitro and in vivo evaluation of other species in this genus to discover new sources of phytochemicals and bioactivities that may be of pharmaceutical importance. 
(2) Several species in the genus are used as traditional medicine to treat various ailments. However, only a few ethnomedicinal applications have been confirmed by scientific studies. Future studies need to be conducted to investigate and verify other ethnomedicinal applications. For example, studies evaluating the anticancer activity of Merremia species are limited in depth and scale. Future studies need to evaluate the cancer cell cytotoxicity activity of different species in a variety of animal models. Additionally, their ethnomedicinal uses in the treatment of stroke and also as alopecia should be investigated in scientific studies.

(3) There are no reported clinical studies to assess the mechanism of action, efficacy, and safety of reported bioactivities in the genus. Clinical studies would be indispensable to adequately characterize these aspects.

(4) More studies are also needed to determine the exact phyto-compound responsible for the different bioactivities and discuss the relation between mechanisms and compounds.

(5) Although most toxicity studies reported that Merremia species are safe to use, a recent toxicity study reported that chronic administration of $M$. tridentata for a prolonged period may cause damage to vital organs in the body [127]. Studies on toxicology and side effects are indispensable in the study of medicinal plants to determine their safety and optimal dose. More toxicological studies should also be the focus of future research in this genus.

Overall, based on the reports of investigations in the genus, it can be concluded that species in the genus Merremia are a valuable nutritional and medicinal resource with promising therapeutic properties. Although the use of Merremia species as food or medicinal plants is encouraged, the long-term sustainability of the plants should also be considered, and as such destructive harvesting involving whole plants for therapeutic purposes should be discouraged to ensure conservation and sustainable use. Conclusively, future research should focus on comprehensive pharmacokinetics studies, in vivo studies, human clinical studies, and toxicity studies. These will show the clinical efficacy and support their development as therapeutic agents against several diseases.

Supplementary Materials: The following are available online at https://www.mdpi.com/article/10 .3390 / plants10102070/s1, Table S1: The full distribution listing of Merremia species around the world.

Author Contributions: Conceptualization, T.L.O. and F.S.; methodology, T.L.O., A.E.A., C.O., O.A.I.; writing-original draft preparation, T.L.O., A.E.A., C.O., O.A.I., O.D.S.; writing-review and editing, T.L.O., A.E.A., C.O., O.A.I.,O.D.S., F.S.; Supervision, F.S. All authors have read and agreed to the published version of the manuscript.

Funding: This research received no external funding.

Conflicts of Interest: The authors declare that there are no conflicts of interest.

$\begin{array}{ll}\text { Abbreviations } \\ \text { A549 } & \text { Lung Carcinoma } \\ \text { ABTS } & \text { 2,2'-azino-bis (3-ethylbenzothiazoline-6-sulfonic acid) } \\ \text { ACE-2 } & \text { Angiotensin-Converting Enzyme 2 } \\ \text { ACEI } & \text { Angiotensin-Converting-Enzyme Inhibitory } \\ \text { AGS } & \text { Stomach Cancer Cells } \\ \text { APFME } & \text { Aqueous Portion of Fractionated Methanol Extract } \\ \text { BHA } & \text { Butylated Hydroxyanisole } \\ \text { BW } & \text { Body Weight } \\ \text { CAFDs } & \text { Caffeic Acid Derivatives } \\ \text { COLO 320 } & \text { Colon Cancer Cells } \\ \text { DM } & \text { Dry Matter } \\ \text { DMSO } & \text { Dimethyl sulfoxide } \\ \text { DPPH } & \text { 1,1-diphenyl-2-picrylhydrazyl } \\ \text { DU-145 } & \text { Prostate Carcinoma }\end{array}$




\begin{tabular}{ll} 
EDTA & Ethylenediaminetetraacetic Acid \\
ESRD & End-Stage Renal Disease \\
EGFR & Epidermal Growth Factor Receptor \\
ELISA & Enzyme-Linked Immunosorbent Assay \\
FITC & Fluorescein Isothiocyanate-Conjugated \\
FRAP & Ferric Reducing Antioxidant Power \\
GAE & Gallic Acid Equivalents \\
HPMC & Hydroxypropylmethylcellulose \\
IP & Intraperitoneally \\
KB & Mouth Carcinoma \\
LPS & Lipopolysaccharide \\
MCF-7 & Breast Cancer Cells \\
MIA-PaCa-2 & Pancreas Carcinoma \\
MIC & Minimum Inhibitory Concentration \\
MTT & 3-(4,5-Dimethylthiazol-2-yl)-2,5-diphenyltetrazolium bromide \\
Na CMC & Sodium Carboxymethylcellulose \\
OH• & Hydroxyl Radicals \\
PI & Propidium Iodide \\
PO & Per os \\
RP-HPLC & Reverse Phase-High Performance Liquid Chromatography \\
TBARS & Thiobarbituric Acid Reactive Substances \\
TGA-capped & Thioglycolic Acid-Capped Cadmium Telluride Quantum Dots \\
-CdTe QDs & \\
TNF- $\alpha$ & Tumour Necrosis Factor Alpha \\
UPLC-MS & Ultra Performance Liquid Chromatography-Tandem Mass Spectrometry \\
/MS & \\
USDA & United State Department of Agriculture \\
VERO & Monkey Normal Kidney Epithelial Cells \\
WFO & World Flora Online \\
WHO & World Health Organization \\
WoS & Web of Science \\
& \\
\hline &
\end{tabular}

\section{References}

1. Cos, P.; Vlietinck, A.J.; Berghe, D.V.; Maes, L. Anti-infective potential of natural products: How to develop a stronger in vitro "proof-of-concept. " J. Ethnopharmacol. 2006, 106, 290-302. [CrossRef]

2. Olukoya, B.M.; Sakpere, A.M.A.; Adetunji, A.E. Phytochemical, proximate and elemental analysis of Clerodendrum volubile (Lamiaceae) at pre-and post-flowering stages. Phytol. Balc. 2018, 24, 45-49.

3. Vasu Babu, A.; Sujatha, K.; Srikanaka Valli, A.; Narayana, K.J.P.; Hari Babu, B.; Satyanarayana, P.V.V. Anticancer and antiinflammatory activities of extracts of Merremia emerginata. Biosci. Biotechnol. Res. Asia 2009, 6, 835-838.

4. Luciardi, M.C.; Pérez Hernández, M.V.; Muruaga, N.; Bardón, A.; Arena, M.E.; Cartagena, E. Volatiles from subtropical Convolvulaceae that interfere with bacterial cell-to-cell communication as potential antipathogenic drugs. Evid. Based Complement. Altern. Med. 2016, 2016, 1-8. [CrossRef] [PubMed]

5. Zhou, Y.; He, Y.J.; Wang, Z.J.; Hu, B.Y.; Xie, T.Z.; Xiao, X.; Zhou, Z.S.; Sang, X.Y.; Luo, X.D. A review of plant characteristics, phytochemistry and bioactivities of the genus Glechoma. J. Ethnopharmacol. 2021, 271, 113830. [CrossRef] [PubMed]

6. Siebert, F.; Dreber, N. Forb ecology research in dry African savannas: Knowledge, gaps, and future perspectives. Ecol. Evol. 2019, 9, 7875-7891. [CrossRef] [PubMed]

7. Bond, W.J.; Parr, C.L. Beyond the forest edge: Ecology, diversity and conservation of the grassy biomes. Biol. Conserv. 2010, 143, 2395-2404. [CrossRef]

8. Adetunji, A.E. Physiological and Biochemical Investigations into the Reinvigoration of Deteriorated Brassica oleracea L. (Cabbage) and Lactuca sativa L. (Lettuce) Seeds with Antioxidants and Inorganic Salt Solutions; University of KwaZulu-Natal: Durban, South Africa, 2021.

9. Staples, G. A Checklist of Merremia (Convolvulaceae) in Australasia and the Pacific. Gard. Bull. Singapore 2010, 61, 483-522.

10. Demissew, S. A synopsis of the genus Merremia (Convolvulaceae) in the Flora of Ethiopia and Eritrea. Kew Bull. 2001, 56, 931-943. [CrossRef]

11. Simoes, A.R.; Culham, A.; Carine, M. Resolving the unresolved tribe: A molecular phylogenetic framework for the Merremieae (Convolvulaceae). Bot. J. Linn. Soc. 2015, 179, 374-387. [CrossRef]

12. Pereda-Miranda, R.; Rosas-Ramírez, D.; Castañeda-Gómez, J. Resin glycosides from the Morning Glory Family. In Fortschritte der Chemie Organischer Naturstoffe = Progress in the Chemistry of Organic Natural Products. Progres Dans la Chimie des Substances 
Organiques Naturelles; Kinghorn, A.D., Falk, H., Gibbons, S., Kobayashi, J., Eds.; Springer: Vienna, Austria, 2010; Volume 92, pp. 77-153. ISBN 9783211996614.

13. Ono, M. Resin glycosides from Convolvulaceae plants. J. Nat. Med. 2017, 71, 591-604. [CrossRef]

14. Schimming, T.; Jenett-Siems, K.; Mann, P.; Tofern-Reblin, B.; Milson, J.; Johnson, R.W.; Deroin, T.; Austin, D.F.; Eich, E. Calystegines as chemotaxonomic markers in the Convolvulaceae. Phytochemistry 2005, 66, 469-480. [CrossRef]

15. Jenett-Siems, K.; Weigl, R.; Böhm, A.; Mann, P.; Tofern-Reblin, B.; Ott, S.C.; Ghomian, A.; Kaloga, M.; Siems, K.; Witte, L.; et al. Chemotaxonomy of the pantropical genus Merremia (Convolvulaceae) based on the distribution of tropane alkaloids. Phytochemistry 2005, 66, 1448-1464. [CrossRef]

16. Sowndhararajan, K.; Chin, N.L. Antioxidant and anti-ulcer effects of ethyl acetate fraction of Merremia tridentata (L.) Hallier, F. Root. Agric. Agric. Sci. Procedia 2014, 2, 406-414. [CrossRef]

17. Ediriweera, E.; Ratnasooriya, W. A review on herbs used in treatment of diabetes mellitus by Sri Lankan ayurvedic and traditional physicians. Ayurveda 2009, 30, 373-391.

18. Yuvaraj, B.; Sathish, M. In Vitro and In Vivo antidiabetic activity on leaves of Merremia hederacea (Burm. f.) Hallier, F. Int. J. Green Pharm. 2019, 13, 384-391.

19. Perez, K.J.; Jose, M.; Aranico, E.; Madamba, M.R. Phytochemical and antibacterial properties of the ethanolic leaf extract of Merremia peltata (L.) Merr. and Rubus SPP. Adv. Environ. Biol. 2015, 9, 50-56.

20. Akter, S.; Jahan, I.; Riniara Khatun, M.; Forhad Khan, M.; Arshad, L.; Haque, M.A. Pharmacological insights into Merremia vitifolia (Burm.f.) Hallier f. leaf for its antioxidant, thrombolytic, anti-arthritic and anti-nociceptive potential. Biosci. Rep. 2021, 20203022. [CrossRef] [PubMed]

21. Rameshkumar, A.; Sivasudha, T.; Jeyadevi, R.; Sangeetha, B.; Ananth, D.A.; Aseervatham, G.S.B.; Nagarajan, N.; Renganathan, R.; Kathiravan, A. In vitro antioxidant and antimicrobial activities of Merremia emarginata using thio glycolic acid-capped cadmium telluride quantum dots. Colloids Surf. B Biointerfaces 2013, 101, 74-82. [CrossRef] [PubMed]

22. Amzad Hossain, M.; Shah, M.D. A study on the total phenols content and antioxidant activity of essential oil and different solvent extracts of endemic plant Merremia borneensis. Arab. J. Chem. 2015, 8, 66-71. [CrossRef]

23. Nahar, M.N.; Acharzo, A.K.; Rahaman, M.S.; Zabeen, I.A.; Haque, S.; Islam, M.A. Phytochemical screening and antioxidant, analgesic, and anthelmintic effect of ethanolic extract of Merremia umbellata stems. Clin. Phytoscience 2020, 6, 86. [CrossRef]

24. Geaney, F.; Scutaru, C.; Kelly, C.; Glynn, R.W.; Perry, I.J. Type 2 Diabetes Research Yield, 1951-2012: Bibliometrics Analysis and Density-Equalizing Mapping. PLoS ONE 2015, 10, e0133009. [CrossRef]

25. Ellegaard, O.; Wallin, J.A. The bibliometric analysis of scholarly production: How great is the impact? Scientometrics 2015, 105, 1809-1831. [CrossRef]

26. Ekundayo, T.C.; Okoh, A.I. A global bibliometric analysis of Plesiomonas-related research (1990-2017). PLoS ONE 2018, 13, e0207655. [CrossRef]

27. Olisah, C.; Adams, J.B. Analysing 70 years of research output on South African estuaries using bibliometric indicators. Estuar. Coast. Shelf Sci. 2021, 252, 107285. [CrossRef]

28. Olisah, C.; Adams, J.B. Systematic mapping of organophosphate contaminant (OPC) research trends between 1990 and 2018. Environ. Geochem. Health 2020, 42, 3481-3505. [CrossRef] [PubMed]

29. Smith, H.H.; Idris, O.A.; Maboeta, M.S. Global trends of green pesticide research from 1994 to 2019: A bibliometric analysis. J. Toxicol. 2021, 2021. [CrossRef]

30. Stefanović, S.; Krueger, L.; Olmstead, R.G. Monophyly of the Convolvulaceae and circumscription of their major lineages based on DNA sequences of multiple chloroplast loci. Am. J. Bot. 2002, 89, 1510-1522. [CrossRef] [PubMed]

31. Stefanović, S.; Austin, D.F.; Olmstead, R.G. Classification of Convolvulaceae: A phylogenetic approach. Syst. Bot. 2003, 28, 791-806. [CrossRef]

32. The Plant List 2013. Available online: http:/ /www.theplantlist.org/tpl1.1/search?q=Merremia (accessed on 3 March 2021).

33. Sosef, M.S.M.; Gereau, R.E.; Janssens, S.B.; Kompanyi, M.; Simões, A.R. A curious new species of Xenostegia (Convolvulaceae) from Central Africa, with remarks on the phylogeny of the genus. Syst. Bot. 2019, 44, 405-414. [CrossRef]

34. Fourie, R. Merremia palmate. Available online: https://www.inaturalist.org/observations/37152427 (accessed on 3 March 2021).

35. Thierrycordenos. Merremia pterygocaulos. Available online: https://www.inaturalist.org/photos/101455002 (accessed on 3 March 2021).

36. Hernández, A.L. Merremia platylphylla. Available online: https://www.inaturalist.org/photos/112408159 (accessed on 3 March 2021).

37. Cock, M. Merremia peltata. Available online: https:/ /www.cabi.org/isc/datasheet/33476\#toPictures (accessed on 3 March 2021).

38. Convolvulaceae Unlimited. Merremia emarginata. Available online: http://tropical.theferns.info/image.php?id=Merremia+ emarginata (accessed on 3 March 2021).

39. Wan-hsuan. Merremia gemella. Available online: https://www.inaturalist.org/observations/32514537 (accessed on 3 March 2021).

40. Global Biodiversity Information Facility (GBIF). Merremia distribution map. Available online: https://www.gbif.org/species/29 28639 (accessed on 3 March 2021).

41. Thet, K.K.; Zar, K.; Lae, W.; Phyo, H.W.; Khin, A.N. Nutritional values and antioxidant activity analysis of Merremia emarginata (Burm.F). 3rd Myanmar Korea Conf. Res. J. 2020, 3, 1549-1555. 
42. Austin, D.F. Merremia dissecta (Convolvulaceae): Condiment, medicine, ornamental, and weed-A review. Econ. Bot. 2007, 61, 109-120. [CrossRef]

43. Catarino, L.; Romeiras, M.M.; Bancessi, Q.; Duarte, D.; Faria, D.; Monteiro, F.; Moldão, M. Edible leafy vegetables from West Africa (Guinea-Bissau): Consumption, trade and food potential. Foods 2019, 8, 493. [CrossRef] [PubMed]

44. Nunes, A.T.; Cabral, D.L.V.; Amorim, E.L.C.; Dos Santos, M.V.F.; Albuquerque, U.P. Plants used to feed ruminants in semi-arid Brazil: A study of nutritional composition guided by local ecological knowledge. J. Arid. Environ. 2016, 135, 96-103. [CrossRef]

45. Aschfalk, A.; Steingass, H.; Müller, W.; Drochner, W. Merremia tridentata as a supplementary feed to the grass Panicum maximum for young West African Dwarf Sheep. Trop. Anim. Health Prod. 2002, 34, 145-150. [CrossRef] [PubMed]

46. Galat-Luong, A.; Nizinski, J.J.; Galat, G. Diet preferences of a Western giant's (Lord Derby's) eland group in a Sahelian dry habitat. Anim. Biol. 2011, 61, 485-492. [CrossRef]

47. Rajendran, D.; Balakrishnan, V. Diet composition, biomass yield and mineral contents of vegetation in native tract of mecheri sheep. Anim. Nutr. Feed Technol. 2012, 12, 63-71.

48. Herrero, M.; Thornton, P.K. Livestock and global change: Emerging issues for sustainable food systems. Proc. Natl. Acad. Sci. USA 2013, 110, 20878-20881. [CrossRef]

49. International Atomic Energy Agency (IAEA). Improving Animal Productivity by Supplementary Feeding of Multinutrient Blocks, Controlling Internal Parasites and Enhancing Utilization of Alternate Feed Resources; IAEA: Vienna, Austria, 2006; pp. 1-280, publication prepared under the framework of an RCA project with technical support of the Joint FAO/IAEA Programme of Nuclear Techniques in Food and Agriculture; Available online: https://www-pub.iaea.org/MTCD/publications/PDF/te_1495_web.pdfx. (accessed on 17 June 2021).

50. Pandey, M.M.; Rastogi, S.; Rawat, A.K.S. Indian traditional ayurvedic system of medicine and nutritional supplementation. Evid. Based Complement. Altern. Med. 2013, 2013. [CrossRef]

51. Sachithanandam, P.; Parkavi, S.; Ganesh, P.; Swaminathan, C. Phytochemical analysis, antibacterial activity and antioxidant activity of leaf extracts of Merremia emarginata (Burm. F.). Int. J. Pharm. Sci. Res. 5214 IJPSR 2020, 11, 5214-5218. [CrossRef]

52. Arunachalam, K.; Parimelazhagan, T. Antidiabetic activity of aqueous root extract of Merremia tridentata (L.) Hall. f. in streptozotocin-induced-diabetic rats. Asian Pac. J. Trop. Med. 2012, 5, 175-179. [CrossRef]

53. Bidkar, A.A.; Sherje, A.P.; Gujar, K.N.; Bagul, U.S.; Miniyar, P.B.; Aphale, S.A. Phytochemical and pharmacological investigation of extracts of Merremia tridentata Linn. (Convolvulaceae). J. Nat. Remedies 2009, 9, 79-84. [CrossRef]

54. Sowndhararajan, K.; Joseph, J.M.; Arunachalam, K.; Manian, S. Evaluation of Merremia tridentata (L.) Hallier f. for in vitro antioxidant activity. Food Sci. Biotechnol. 2010, 19, 663-669. [CrossRef]

55. Kamalutheen, M.; Gopalakrishnan, S.; Syed Ismail, T. Anti-inflammatory and anti-arthritic activities of Merremia tridentata (L.) Hall. f. J. Chem. 2009, 6, 943-948. [CrossRef]

56. Ratnadewi, A.A.I.; Wahyudi, L.D.; Rochman, J.; Susilowati; Nugraha, A.S.; Siswoyo, T.A. Revealing anti-diabetic potency of medicinal plants of Meru Betiri National Park, Jember-Indonesia. Arab. J. Chem. 2020, 13, 1831-1836. [CrossRef]

57. Marchianti, A.C.N.; Sakinah, E.N.; Elfiah, U.; Putri, N.K.S.; Wahyuliswari, D.I.; Maulana, M.; Ulfa, E.U. Gel formulations of Merremia mammosa (Lour.) accelerated wound healing of the wound in diabetic rats. J. Tradit. Complement. Med. 2021, 11, 38-45. [CrossRef]

58. Alen, Y.; Sari, P.; Aldi, Y.; Yulianis; Nakajima, S.; Baba, N.; Djamaan, A. Extraction, fractionation and cytotoxicity test of Merremia peltata (L.)Merr., (Fam. Convolvulaceae) Leaves. Der. Pharm. Lett. 2016, 8, 48-52.

59. Hossain, M.A.; Shah, M.D.; Sang, S.V.; Sakari, M. Chemical composition and antibacterial properties of the essential oils and crude extracts of Merremia borneensis. J. King Saud Univ. Sci. 2012, 24, 243-249. [CrossRef]

60. Li, Q.; Yuan, M.; Li, J.; Wei, D.; Mei, S.; Cui, T.; Wang, J.; Zhu, Z.; Dong, X.; Wan, J. Structure elucidation and NMR assignment of two new eudesmane derivatives from Merremia yunnanensis. Magn. Reson. Chem. 2019, 57, 934-938. [CrossRef]

61. Yuan, M.; Liu, B.; Ding, Q.; Zhang, X.; Wei, D.; Mei, S.; Li, R.; Wan, J.; Li, Q. A new bicyclic sesquiterpenoid from Merremia yunnanensis. Biochem. Syst. Ecol. 2020, 89, 103964. [CrossRef]

62. Rivera, D.E.; Ocampo, Y.C.; Castro, J.P.; Caro, D.; Franco, L.A. Antibacterial activity of Physalis angulata L., Merremia umbellata L., and Cryptostegia grandiflora Roxb. Ex R.Br.-medicinal plants of the Colombian Northern Coast. Orient. Pharm. Exp. Med. 2015, 15, 95-102. [CrossRef]

63. Awang-Jamil, Z.; Aminuddin, M.F.; Zaidi, B.Q.; Basri, A.M.; Ahmad, N.; Taha, H. Phytochemicals and antimicrobial analysis of selected medicinal plants from Brunei Darussalam. Biodiversitas 2021, 22, 601-606. [CrossRef]

64. Rameshkumar, A.; Sivasudha, T.; Jeyadevi, R.; Sangeetha, B.; Smilin Bell Aseervatham, G.; Maheshwari, M. Profiling of phenolic compounds using UPLC-Q-TOF-MS/MS and nephroprotective activity of Indian green leafy vegetable Merremia emarginata (Burm. f.). Food Res. Int. 2013, 50, 94-101. [CrossRef]

65. Kim, H.R.; Chung, S.Y.; Jeong, Y.H.; Go, E.J.; Han, A.R.; Kim, N.H.; KyungSung, M.; Song, G.; Jang, J.O.; Nam, J.W.; et al. P-glycoprotein inhibitory activity of Indonesian medicinal plants in human breast cancer cells. Nat. Prod. Sci. 2004, 10, 268-271.

66. Pavithra, P.S.; Janani, V.S.; Charumathi, K.H.; Indumathy, R.; Potala, S.; Verma, R.S. Antibacterial activity of plants used in Indian herbal medicine. Int. J. Green Pharm. 2010, 4, 22-28. [CrossRef]

67. Ch, D.; Prasad, R.Y.; Babu, S.P.; Gananadhamu, S. Biological screening and phytochemical investigation of Merremia emarginata leaves. Int. J. Pharm. Sci. Res. 2021, 12, 507-514. [CrossRef] 
68. Anioł, M.; Świderska, A.; Stompor, M.; Żołnierczyk, A.K. Antiproliferative activity and synthesis of 8-prenylnaringenin derivatives by demethylation of 7-O-and 4'-O-substituted isoxanthohumols. Med. Chem. Res. 2012, 21, 4230-4238. [CrossRef]

69. Paulo, F.; Santos, L. Encapsulation of the Antioxidant tyrosol and characterization of loaded microparticles: An integrative approach on the study of the polymer-carriers and loading contents. Food Bioprocess Technol. 2020, 13, 764-785. [CrossRef]

70. Adetunji, A.E.; Sershen, N.; Varghese, B.; Pammenter, N. Effects of exogenous application of five antioxidants on vigour, viability, oxidative metabolism and germination enzymes in aged cabbage and lettuce seeds. S. Afr. J. Bot. 2021, 137, 85-97. [CrossRef]

71. Adetunji, A.E.; Sershen, N.; Varghese, B.; Pammenter, N.W. Effects of inorganic salt solutions on vigour, viability, oxidative metabolism and germination enzymes in aged cabbage and lettuce seeds. Plants 2020, 9, 1164. [CrossRef]

72. Lee, J.; Han, S.I.; Yun, J.H.; Kim, J.H. Quercetin 3-O-glucoside suppresses epidermal growth factor-induced migration by inhibiting EGFR signaling in pancreatic cancer cells. Tumor Biol. 2015, 36, 9385-9393. [CrossRef] [PubMed]

73. Rahman, F.; Tabrez, S.; Ali, R.; Alqahtani, A.S.; Ahmed, M.Z.; Rub, A. Molecular docking analysis of rutin reveals possible inhibition of SARS-CoV-2 vital proteins. J. Tradit. Complement. Med. 2021, 11, 173-179. [CrossRef] [PubMed]

74. Wang, W.Q.; Song, W.B.; Lan, X.J.; Huang, M.; Xuan, L.J. Merremins A-G, resin glycosides from Merremia hederacea with multidrug resistance reversal activity. J. Nat. Prod. 2014, 77, 2234-2240. [CrossRef] [PubMed]

75. Yan, J.; Bi, H.-H.; Liu, Y.-Z.; Zhang, M.; Zhou, Z.-Y.; Tan, J.-W. Phenolic compounds from Merremia umbellata subsp.orientalis and their allelopathic effects on Arabidopsis seed germination. Molecules 2010, 15, 8241-8250. [CrossRef]

76. Adem, S.; Eyupoglu, V.; Sarfraz, I.; Rasul, A.; Zahoor, A.F.; Ali, M.; Abdalla, M.; Ibrahim, I.M.; Elfiky, A.A. Caffeic acid derivatives (CAFDs) as inhibitors of SARS-CoV-2: CAFDs-based functional foods as a potential alternative approach to combat COVID-19. Phytomedicine 2020, 153310. [CrossRef]

77. Gasparotto, A.J.; Tolouei, S.E.L.; Lívero, F.A.; Gasparotto, F.; Boeing, T.; De Souza, P. Natural agents modulating ACE-2: A review of compounds with potential against SARS-CoV-2 infections. Curr. Pharm. Des. 2021, 27. [CrossRef]

78. Gaikwad, S.B.; Krishna Mohan, G.; Rani, M.S. Phytochemicals for diabetes management. Pharm. Crop. 2014, 5, 11-28. [CrossRef]

79. Angappan, R.; Devanesan, A.A.; Thilagar, S. Diuretic effect of chlorogenic acid from traditional medicinal plant Merremia emarginata (Burm. F.) and its by product hippuric acid. Clin. Phytoscience 2018, 4, 29. [CrossRef]

80. Santos Júnior, H.M.; Lopes, K.C.; Alves, D.S.; Carvalho, G.A.; Oliveira, D.F. Ursolic acid and cis-tiliroside produced by Merremia tomentosa affect oviposition of Leucoptera coffeella on coffee plants. Quim. Nova 2018, 41, 302-309. [CrossRef]

81. Jenett-Siems, K.; Siems, K.; Witte, L.; Eich, E. Merrekentrones A-D, Ipomeamarone-like furanosesquiterpenes from Merremia kentrocaulos. J. Nat. Prod. 2001, 64, 1471-1473. [CrossRef]

82. Kitagawa, I.; Ohashi, K.; Baek, N.I.; Sakagami, M.; Yoshikawa, M.; Shibuya, H. Indonesian Medicinal Plants. XIX. Chemical Structures of Four Additional Resin-Glycosides, Mammosides A, B, H1, and H2, from the Tuber of Merremia mammosa (Convolvulaceae). Chem. Pharm. Bull. 1997, 45, 786-794. [CrossRef]

83. García-Argáez, A.; Pérez-Amador, M.; Aguirre-Hernández, E.; Martínez-Vázquez, M. Two new caffeate esters from roots of Merremia tuberosa and M. dissecta. Planta Med. 1999, 65, 678-679. [CrossRef]

84. Kitagawa, I.; Baek, N.I.; Kawashima, K.; Yokokawa, Y.; Yoshikawa, M.; Ohashi, K.; Shibuya, H. Indonesian medicinal plants. XV. Chemical structures of five new resin- glycosides, Merremosides A, B, C, D, and E, from the tuber of Merremia mammosa (Convolvulaceae). Chem. Pharm. Bull. 1996, 44, 1680-1692. [CrossRef]

85. Kitagawa, I.; Baek, N.I.; Yokokawa, Y.; Yoshikawa, M.; Ohashi, K.; Shibuya, H. Indonesian Medicinal Plants. XVI. Chemical structures of four new resin-glycosides, Merremosides F, G, H1, and H2, from the tuber of Merremia mammosa (Convolvulaceae). Chem. Pharm. Bull. 1996, 44, 1693-1699. [CrossRef]

86. Vilakazi, H.; Olasehinde, T.A.; Olaniran, A.O. Chemical characterization, antiproliferative and antioxidant activities of polyunsaturated fatty acid-rich extracts from Chlorella sp. S14. Molecules 2021, 26, 4109. [CrossRef]

87. Chaudhary, S.K.; Sandasi, M.; Makolo, F.; Van Heerden, F.R.; Viljoen, A.M. Aspalathin: A rare dietary dihydrochalcone from Aspalathus linearis (rooibos tea). Phytochem. Rev. 2021, 1-32. [CrossRef]

88. Baskar, A.A.; Al Numair, K.S.; Alsaif, M.A.; Ignacimuthu, S. In vitro antioxidant and antiproliferative potential of medicinal plants used in traditional Indian medicine to treat cancer. Redox Rep. 2012, 17, 145-156. [CrossRef] [PubMed]

89. WHO. Diabetes-WHO-Regional Office for Africa Diabetes-WHO-Regional Office for Africa. Available online: https: / www. afro.who.int/health-topics/diabetes (accessed on 10 July 2021).

90. Muthi'atul Af-Idah, B.; Hanafi, M.; Elya, B. Antioxidant and alpha glucosidase inhibitor screening of Merremia peltata L. as potential traditional treatment for diabetes mellitus. Orig. Artic. Pharm. J. 2021, 13, 902-908. [CrossRef]

91. Corona, G.; Pizzocaro, A.; Vena, W.; Rastrelli, G.; Semeraro, F.; Isidori, A.M.; Pivonello, R.; Salonia, A.; Sforza, A.; Maggi, M. Diabetes is most important cause for mortality in COVID-19 hospitalized patients: Systematic review and meta-analysis. Rev. Endocr. Metab. Disord. 2021, 22, 275-296. [CrossRef] [PubMed]

92. Huang, I.; Lim, M.A.; Pranata, R. Diabetes mellitus is associated with increased mortality and severity of disease in COVID-19 pneumonia-A systematic review, meta-analysis, and meta-regression: Diabetes and COVID-19. Diabetes Metab. Syndr. Clin. Res. Rev. 2020, 14, 395-403. [CrossRef]

93. Gandhi, G.R.; Sasikumar, P. Antidiabetic effect of Merremia emarginata Burm. F. in streptozotocin induced diabetic rats. Asian Pac. J. Trop. Biomed. 2012, 2, 281-286. [CrossRef]

94. Kaladhar, D.S.V.G.K.; Harasreeramulu, S.; Vijaya Rachel, K.; Surekha, C.H. Evaluation of antimicrobial activity of methanolic leaf extracts of selected in vitro and in vivo grown Convolvulaceae members. J. Pure Appl. Microbiol. 2009, 3, 759-767. 
95. Elumalai, E.K.; Ramachandran, M.; Thirumalai, T.; Vinothkumar, P. Antibacterial activity of various leaf extracts of Merremia emarginata. Asian Pac. J. Trop. Biomed. 2011, 1, 406-408. [CrossRef]

96. Diwan, P.D.; Gadhikar, Y.A. Assessment of phytochemical composition and antibacterial activity of different extracts of Barleria prionitis leaves against oral microflora to improve dental hygiene. Asian J. Pharm. Clin. Res. 2012, 5, $182-184$.

97. Yang, C.H.; Tan, D.H.; Hsu, W.L.; Jong, T.T.; Wen, C.L.; Hsu, S.L.; Chang, P.C. Anti-influenza virus activity of the ethanolic extract from Peperomia sui. J. Ethnopharmacol. 2014, 155, 320-325. [CrossRef] [PubMed]

98. WHO Influenza (Seasonal). Available online: https://www.who.int/news-room/fact-sheets/detail/influenza-(seasonal) (accessed on 12 July 2021).

99. Purwitasari, N.; Agil, M.; Studiawan, H. Activity of ethyl acetate fraction of Merremia mammosa Hall. as anti-influenza A (H1N1). Indian J. Forensic Med. Toxicol. 2020, 14, 2070-2073. [CrossRef]

100. Khumalo, G.P.; Van Wyk, B.E.; Feng, Y.; Cock, I.E. A review of the traditional use of Southern African medicinal plants for the treatment of inflammation and inflammatory pain. J. Ethnopharmacol. 2021, 114436. [CrossRef]

101. Shebaby, W.; Saliba, J.; Faour, W.H.; Ismail, J.; El Hage, M.; Daher, C.F.; Taleb, R.I.; Nehmeh, B.; Dagher, C.; Chrabieh, E.; et al In Vivo and In Vitro anti-inflammatory activity evaluation of Lebanese Cannabis sativa L. ssp. indica (Lam.). J. Ethnopharmacol. 2021, 270, 113743. [CrossRef]

102. Purushoth Prabhu, T.; Sudev, S.; Deepak Venkataraman, N.; Clement Atlee, W.; Shrikumar, S. In Vitro antioxidant and antiarthritis activity of extracts and fractions of Merremia emarginata. Der Pharm. Lett. 2014, 6, 227-231.

103. Raja, S.N.; Carr, D.B.; Cohen, M.; Finnerup, N.B.; Flor, H.; Gibson, S.; Keefe, F.J.; Mogil, J.S.; Ringkamp, M.; Sluka, K.A.; et al. The revised international association for the study of pain, definition of pain: Concepts, challenges, and compromises. Pain 2020, 161, 1976-1982. [CrossRef]

104. Ahmadimoghaddam, D.; Zarei, M.; Mohammadi, S.; Izadidastenaei, Z.; Salehi, I. Bupleurum falcatum L. alleviates nociceptive and neuropathic pain: Potential mechanisms of action. J. Ethnopharmacol. 2021, 273, 113990. [CrossRef] [PubMed]

105. Mosquera, D.M.G.; Ortega, Y.H.; Quero, P.C.; Martínez, R.S.; Pieters, L. Antiurolithiatic activity of Boldoa purpurascens aqueous extract: An in vitro and in vivo study. J. Ethnopharmacol. 2020, 253, 112691. [CrossRef] [PubMed]

106. Priya, S.; Arul, S.V. Anti-Urolithiatic activity of medicinal plants and Siddha formulatory medicine: A Review. J. Res. Biomed. Sci. 2020, 3, 7-12. [CrossRef]

107. Neeraja Kamakshi, U.; Ganga Rao, B.; Venkateswara Rao, B. Comparison of in vitro antiurolithiatic activity of Aerva lanata, Sphaeranthus indicus, Merremia emarginata. Res. J. Pharm. Technol. 2017, 10, 1653-1656. [CrossRef]

108. Sharma, V.C.; Kaushik, A.; Dey, Y.N.; Srivastava, B.; Wanjari, M.; Pawar, S.; Chougule, S. Nephroprotective potential of Anogeissus latifolia Roxb. (Dhava) against gentamicin-induced nephrotoxicity in rats. J. Ethnopharmacol. 2021, 273, 114001. [CrossRef]

109. Jabeen, Q.; Aslam, N. Hypotensive, angiotensin converting enzyme (ACE) inhibitory and diuretic activities of the aqueousmethanol extract of Ipomoea reniformis. Iran. J. Pharm. Res. 2013, 12, 769-776. [CrossRef] [PubMed]

110. Rajoo, A.; Ramanathan, S.; Mansor, S.M.; Sasidharan, S. Formulation and evaluation of wound healing activity of Elaeis guineensis Jacq leaves in a Staphylococcus aureus infected Sprague Dawley rat model. J. Ethnopharmacol. 2021, 266, 113414. [CrossRef] [PubMed]

111. Jarić, S.; Kostić, O.; Mataruga, Z.; Pavlović, D.; Pavlović, M.; Mitrović, M.; Pavlović, P. Traditional wound-healing plants used in the Balkan region (Southeast Europe). J. Ethnopharmacol. 2018, 211, 311-328. [CrossRef] [PubMed]

112. Sakinah, E.N.; Ulfa, E.U.; Marchianti, A.C.N. The effectiveness of Merremia mammosa (Lour.) extract fractions as diabetic wound healers on diabetic rat model. Hiroshima J. Med. Sci. 2018, 67, 70-77.

113. Wei, X.; Zhao, Z.; Zhong, R.; Tan, X. A comprehensive review of herbacetin: From chemistry to pharmacological activities. J. Ethnopharmacol. 2021, 279, 114356. [CrossRef]

114. Idris, O.A.; Wintola, O.A.; Afolayan, A.J. Phytochemical and antioxidant activities of Rumex crispus L. in treatment of gastrointestinal helminths in Eastern Cape Province, South Africa. Asian Pac. J. Trop. Biomed. 2017, 7, 1071-1078. [CrossRef]

115. Gomathi, D.; Kalaiselvi, M.; Ravikumar, G.; Devaki, K.; Uma, C. GC-MS analysis of bioactive compounds from the whole plant ethanolic extract of Evolvulus alsinoides (L.) L. J. Food Sci. Technol. 2015, 52, 1212-1217. [CrossRef]

116. Babu, A.V.; Rao, R.S.C.; Kumar, K.G.; Babu, B.H.; Satyanarayana, P.V.V. Biological activity of Merremia emarginata crude extracts in different solvents. Res. J. Med. Plant 2009, 3, 134-140. [CrossRef]

117. Joshi, R.; Mishra, P.; Patni, V. Untapped ornamental vines of Convolvulaceae-potential source of antioxidants. Int. J. Pharm. Sci. Rev. Res. 2016, 36, 120-123.

118. Thamsermsang, O.; Akarasereenont, P.; Laohapand, T.; Panich, U. IL-1 $\beta$-induced modulation of gene expression profile in human dermal fibroblasts: The effects of Thai herbal Sahatsatara formula, piperine and gallic acid possessing antioxidant properties. BMC Complement. Altern. Med. 2017, 17, 32. [CrossRef] [PubMed]

119. Makajanma, M.M.; Taufik, I.; Faizal, A. Antioxidant and antibacterial activity of extract from two species of mosses: Leucobryum aduncum and Campylopus schmidii. Biodiversitas 2020, 21, 2751-2758. [CrossRef]

120. Benelli, G.; Pavela, R.; Rakotosaona, R.; Nzekoue, F.K.; Canale, A.; Nicoletti, M.; Maggi, F. Insecticidal and mosquito repellent efficacy of the essential oils from stem bark and wood of Hazomalania voyronii. J. Ethnopharmacol. 2020, 248, 112333. [CrossRef] [PubMed]

121. Ashokkumar, K.; Murugan, M.; Dhanya, M.K.; Warkentin, T.D. Botany, traditional uses, phytochemistry and biological activities of Cardamom [Elettaria cardamomum (L.) Maton]—A critical review. J. Ethnopharmacol. 2020, 246, 112244. [CrossRef] 
122. Oliveira, P.V.; Ferreira, J.C.; Moura, F.S.; Lima, G.S.; De Oliveira, F.M.; Oliveira, P.E.S.; Conserva, L.M.; Giulietti, A.M.; Lemos, R.P.L. Larvicidal activity of 94 extracts from ten plant species of Northeastern of Brazil against Aedes aegypti L. (Diptera: Culicidae). Parasitol. Res. 2010, 107, 403-407. [CrossRef]

123. Azarudeen, R.M.S.T.; Govindarajan, M.; AlShebly, M.M.; AlQahtani, F.S.; Amsath, A.; Senthilmurugan, S.; Vijayan, P.; Benelli, G. Size-controlled biofabrication of silver nanoparticles using the Merremia emarginata leaf extract: Toxicity on Anopheles stephensi, Aedes aegypti and Culex quinquefasciatus (Diptera: Culicidae) and non-target mosquito predators. J. Asia Pac. Entomol. 2017, 20, 359-366. [CrossRef]

124. Kamble, S.; Kamble, V.S. Antiinflammatory activity of the methanolic root extract of Merremia tridentata (L.) Hall. F. J. Pharmacogn. Phytochem. 2017, 6, 470-471.

125. Priya, P.; Arul An, C.N.; Gausunnisha, T.; Rajalakshmi, R.; Roselin, A.; Grandhi, U. Study of analgesic activity of Merremia emarginata (BURM. F) HALLIER F. Int. J. Pharm. Biol. Sci. 2012, 2, 299-302.

126. Brito, L.B.; Silva Filho, G.B.; Chaves, H.A.S.; Nascimento, A.L.O.; Braga, T.C.; Pfister, J.; Correa, F.R.; Mendonça, F.S. Spontaneous and experimental poisoning by Merremia macrocalyx (Convolvulaceae) in cattle1. Pesqui. Vet. Bras. 2019, 39, 447-453. [CrossRef]

127. Adebiyi, O.A.; Danladi, A.A.; Onyike, E.; James, D.B. Acute and chronic toxicity studies of ethanol Leaf extract of Merremia tridentata (Linn) Hallier, F. Eur. J. Adv. Chem. Res. 2021, 2, 14-20. [CrossRef]

128. Miller, J.S. The Global Importance of Plants as Sources of Medicines and the Future Potential of Chinese Plants. In Drug Discovery and Traditional Chinese Medicine; Springer: Boston, MA, USA, 2001; pp. 33-42.

129. Dzobo, K. The Role of Natural Products as Sources of Therapeutic Agents for Innovative Drug Discovery. In Reference Module in Biomedical Sciences; Elsevier: Amsterdam, The Netherlands, 2021. 